Check for updates

Cite this: Chem. Soc. Rev., 2021, 50,6541

Received 11th December 2020

DOI: $10.1039 / \mathrm{d} 0 \operatorname{cs} 01541 \mathrm{e}$

rsc.li/chem-soc-rev

\title{
Atomically precise graphene nanoribbons: interplay of structural and electronic properties
}

\author{
R. S. Koen Houtsma, (D) * Joris de la Rie (D) and Meike Stöhr (D) *
}

\begin{abstract}
Graphene nanoribbons hold great promise for future applications in nanoelectronic devices, as they may combine the excellent electronic properties of graphene with the opening of an electronic band gap not present in graphene but required for transistor applications. With a two-step on-surface synthesis process, graphene nanoribbons can be fabricated with atomic precision, allowing precise control over width and edge structure. Meanwhile, a decade of research has resulted in a plethora of graphene nanoribbons having various structural and electronic properties. This article reviews not only the onsurface synthesis of atomically precise graphene nanoribbons but also how their electronic properties are ultimately linked to their structure. Current knowledge and considerations with respect to precursor design, which eventually determines the final (electronic) structure, are summarized. Special attention is dedicated to the electronic properties of graphene nanoribbons, also in dependence on their width and edge structure. It is exactly this possibility of precisely changing their properties by fine-tuning the precursor design - offering tunability over a wide range - which has generated this vast research interest, also in view of future applications. Thus, selected device prototypes are presented as well.
\end{abstract}

\section{Introduction}

The first isolation of graphene, a two-dimensional sheet of $\mathrm{sp}^{2}$ hybridized carbon atoms, by Geim and Novoselov ${ }^{1}$ has sparked an active and still growing field of research, in particular within materials science and condensed matter physics. Graphene is expected to host interesting new physics, as well as having

Zernike Institute for Advanced Materials, University of Groningen, Nijenborgh 4, 9747AG, Groningen, The Netherlands. E-mail: r.s.k.houtsma@rug.nl, m.a.stohr@rug.nl

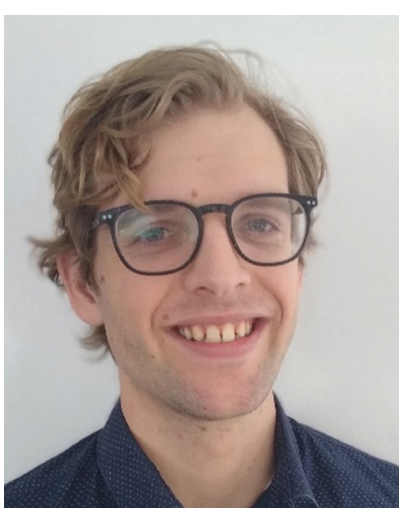

R. S. Koen Houtsma
R. S. Koen Houtsma received his MSc degree in Applied Physics from the University of Groningen in 2019 under the supervision of Prof. Meike Stöhr. Afterwards, he joined the Surface Science group of Prof. Stöhr at the Zernike Institute for Advanced Materials at the University of Groningen as a PhD student. His research focuses on tuning the electronic properties of graphene, for instance by quantum confinement or molecular selfassembly, for electronic and spintronic applications. desirable, so far not available, electronic and mechanical properties. ${ }^{2-4}$ Graphene is a gapless semimetal and when implemented in a field-effect device has an on/off ratio between 2 and $20 .^{5}$ However, for graphene-based materials to fulfil their promise for use in high performance future electronic devices the on/off ratio must be improved by several orders of magnitude, for which a sizeable bandgap is an essential requirement. ${ }^{5}$

By cutting graphene into a quasi-one-dimensional strip, a graphene nanoribbon (GNR), a bandgap can be opened through quantum confinement of the charge carriers. ${ }^{6}$ Consequently, this prospect has spurred significant effort to fabricate and

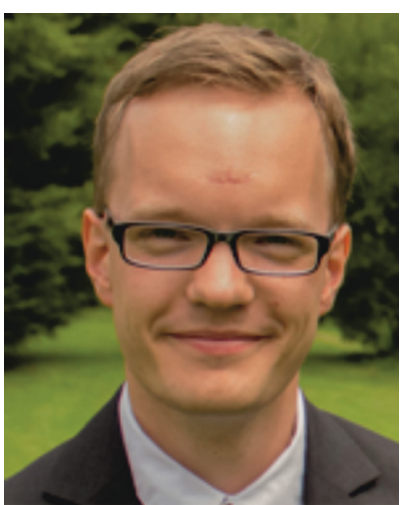

Joris de la Rie
Joris de la Rie has been a PhD student in the group of Prof. Meike Stöhr at the Zernike Institute for Advanced Materials since November 2018. His research focuses on functionalizing graphene through self-assembled networks of organic molecules, and how graphene influences the self-assembly of these molecules. Prior to joining the group of Prof. Meike Stöhr, he obtained his BSc and MSc in Applied Physics from the University of Twente. 
study the properties of GNRs. GNRs can be fabricated by means of, for instance, lithography, ${ }^{7,8}$ chemical synthesis, ${ }^{9-16}$ the unzipping of carbon nanotubes (CNTs) ${ }^{17-20}$ or epitaxial growth on silicon carbide sidewalls. $^{21-27}$ GNRs obtained with such fabrication techniques showed attractive properties such as ballistic transport $^{26,27}$ and spin-split edge states. ${ }^{19}$ However, it has to be noted that the electronic properties of GNRs are extremely sensitive to their exact width and edge termination. For instance, increasing the width of a GNR by a single carbon atom can theoretically alter the band gap by approximately $1 \mathrm{eV}^{28,29}$ In addition, even small amounts of edge disorder can act as sources for scattering, leading to transport limitations. ${ }^{30-32}$ Thus, precise control over the GNR structure is a prerequisite for GNR-based devices.

The first atomically precise GNRs, i.e. GNRs having welldefined edges down to the single atom limit, were synthesized a decade ago using an on-surface bottom-up approach. ${ }^{33-37}$ Since then, GNR on-surface synthesis has been a rapidly growing field of increasing scientific interest. Now, a decade later, a vast catalogue of atomically precise GNRs has been synthesized and the properties have been characterized using various experimental techniques. The increasing scientific knowledge on the subject may produce a feast-like selection of GNRs from which the right one having the suitable properties for the desired future applications is available. For instance, GNRs with band gaps between $0.1 \mathrm{eV}$ and $2-3 \mathrm{eV}$ have been so far synthesized, making them viable for use within a range of electronic devices. In fact, device prototypes have already been fabricated which can reach on/off ratios as high as $10^{5} .{ }^{38}$ In addition, GNRs with a zigzag edge structure are expected to host spin-polarized states, making them interesting for spintronic applications, as well. The precise control over the final GNR structure that is afforded by on-surface synthesis allows the fabrication of GNRs with an unconventional shape, atomically precise doping ${ }^{39}$ or embedding exotic, topological phases, ${ }^{40-42}$ further highlighting the versatility of GNRs.

In this review we will discuss the bottom-up, on-surface synthesis of atomically precise GNRs with a particular view on

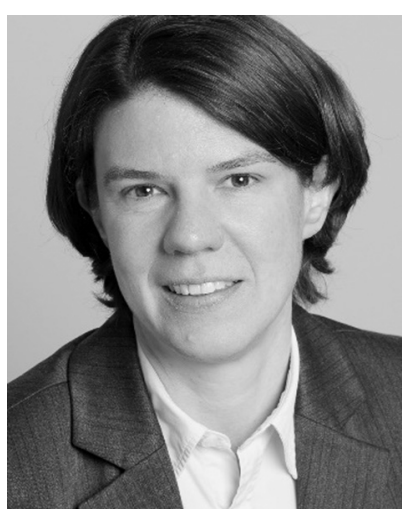

Meike Stöhr
Meike Stöhr is a professor at the Zernike Institute for Advanced Materials of the University of Groningen (Netherlands). She obtained her PhD in Physics from the University of Essen (Germany) in 2002. For postdoctoral training, she moved to the University of Basel (Switzerland), where she also obtained her habilitation in 2008. Her current research focuses on on-surface molecular selfassembly as well as on-surface synthesis and how the (electronic) properties of $2 D$ materials can be tuned by molecular patterning.
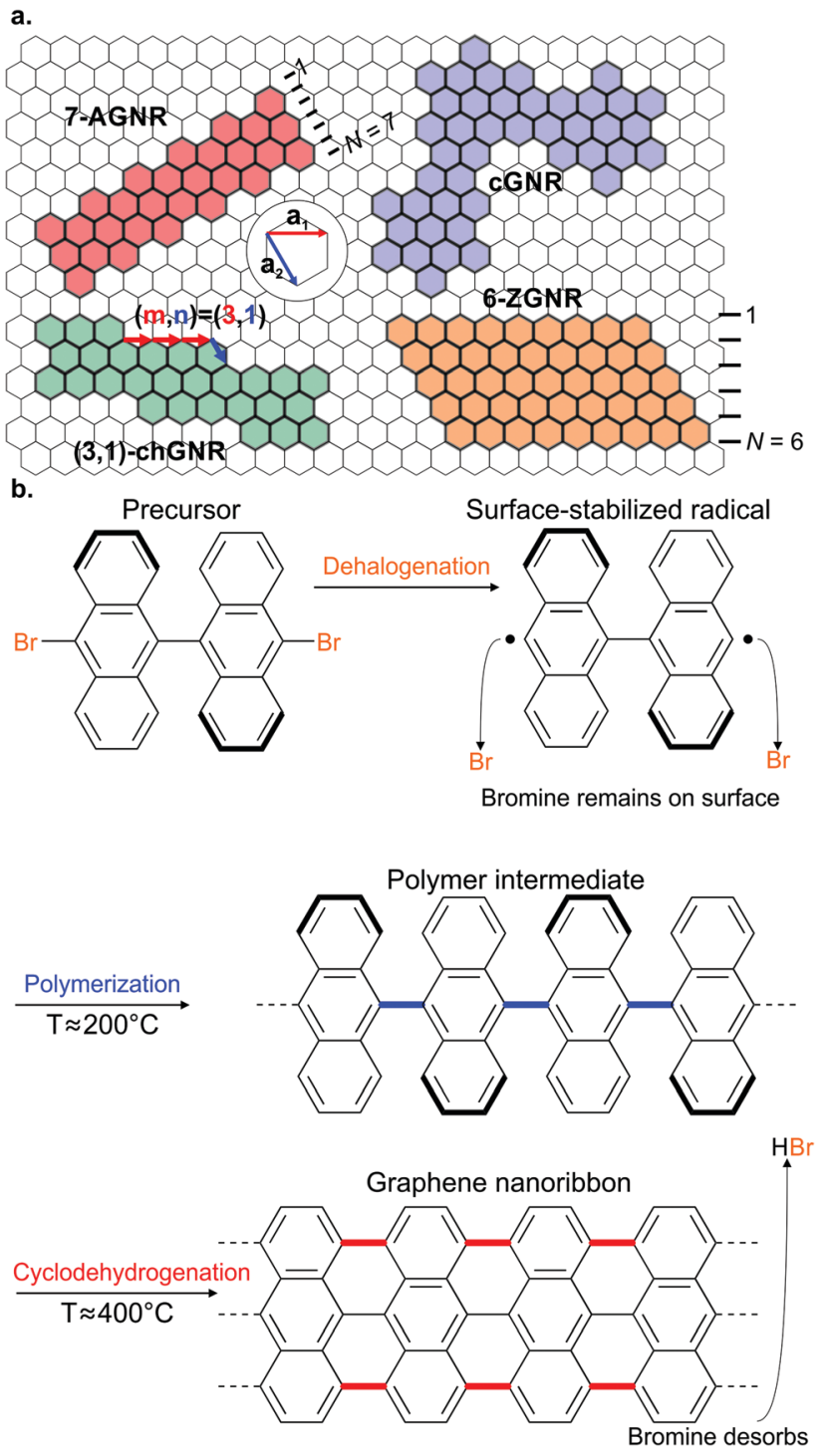

Fig. 1 (a) Various GNR types and their classification. (b) Schematic overview of on-surface GNR fabrication for the case of a 7-AGNR.

their electronic properties. Starting from the basic theory, we will especially focus on the experimental characterization of the electronic properties. Lastly, we will briefly discuss the use of bottom-up fabricated GNRs in devices, specifically GNR-based field-effect transistors. We conclude with a discussion about the challenges that lie ahead and provide an outlook.

\section{On-surface synthesis of graphene nanoribbons}

There are four common types of GNRs: armchair (AGNRs), zigzag (ZGNRs), chiral (chGNRs) and chevron (cGNRs) ones. AGNRS and ZGNRs can be uniquely identified by their width only. For AGNRs, width is typically expressed in the number of dimer lines, whereas for ZGNRs width is expressed according to the number of zigzag lines (Fig. 1a). Thus, please note that a 
7-AGNR (i.e. an AGNR that is seven dimer lines wide) is narrower than a 6-ZGNR.

On the other hand, two parameters are required to uniquely identify chGNRs: their width and edge orientation. The edge orientation is determined by the number of graphene unit cell vectors, $m \mathbf{a}_{1}$ and $n \mathbf{a}_{2}$, along the edge of the chGNRs unit cell (Fig. 1a).

There is no generally agreed upon classification scheme (although such a scheme has been proposed ${ }^{43}$ ) for cGNRs. cGNRs are GNRs with a meandering topology. They can possess a combination of armchair and zigzag edge terminations.

Since the seminal work of Cai et al., ${ }^{37}$ who successfully fabricated the first atomically precise AGNR using on-surface synthesis, most GNRs have been synthesized using the same multistep process based on Ullmann-type coupling and cyclodehydrogenation. The first step involves the evaporation of a halogenated aromatic precursor on a well-defined (mainly single crystal) surface. Second, the halogen substituents are split off in a thermally-induced, surface-assisted Ullmann-type coupling reaction resulting in the formation of a polymer or metal-organic intermediate. Lastly, surface-assisted cyclodehydrogenation converts the polymer into a GNR with welldefined width and edge termination. Below, we will discuss the different steps involved in GNR on-surface synthesis.

\section{Ullmann-type coupling}

Ullmann coupling is a reaction where two aryl halides couple to form a biaryl, catalysed by $\mathrm{Cu} .{ }^{44}$ The effectiveness of Ullmanntype coupling for the rational, bottom-up design of covalently coupled one- or two-dimensional nano-architectures on metallic substrates was shown in pioneering work by Grill et al. ${ }^{45}$ In the past few decades, Ullmann-type coupling has proven to be uniquely useful for the creation of surface-confined zero-dimensional clusters, one-dimensional (1D) chains and two-dimensional
(2D) networks. ${ }^{46-48}$ For the specific purpose of synthesizing GNRs, Ullmann-type coupling is the most ubiquitous reaction for generating the polymer intermediate because it normally does not have side reactions and, if an appropriate molecular precursor is used, the polymer intermediate already has the correct geometry for subsequent cyclodehydrogenation. ${ }^{49}$ Ullmann-type coupling typically occurs in three distinct, time-separated steps: (i) dissociation of the halogen substituents, leaving a surfacestabilized radical (Fig. 1b), (ii) subsequent surface-confined diffusion of the radical and (iii) recombination of the radicals, ${ }^{37,49-53}$ forming a polymer intermediate. Below, we will discuss the design of the precursor and the role of both the substrate and substituted halogen.

Precursor design. A crucial part in fabricating well-defined GNRs via on-surface synthesis is the precursor. Eventually, it determines the polymer that is formed upon Ullmann-type coupling, and thereby also the GNR upon cyclodehydrogenation. Therefore, the precursor used also determines the electronic properties of the GNR.

All precursors used so far are polycyclic aromatic hydrocarbons (PAHs) with at least two halogen substitutions. A large selection of precursors used so far, together with their resulting GNR structures, is given in Fig. 3. The simplest precursors are those with 2-fold rotational symmetry and halogen substitutions in a para position (Fig. 2, blue building blocks). These precursors lead to AGNRs with the same width as the precursor (e.g. the synthesis depicted in Fig. 2b) or a ribbon with periodic width modulation (e.g. 79). Creating wider ribbons following this design strategy requires larger precursors which are more difficult to synthesize (e.g. synthesizing acenes higher than pentacene poses a significant challenge ${ }^{54}$ ) and might not even sublime due to increased mass and intermolecular interactions. ${ }^{55}$ To circumvent this issue, an effective strategy is to design the precursor such that steric a.
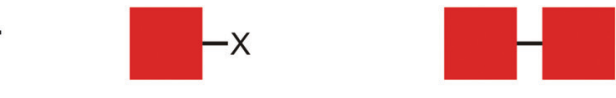

Para
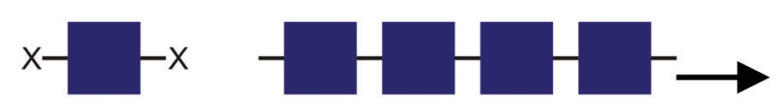

b.
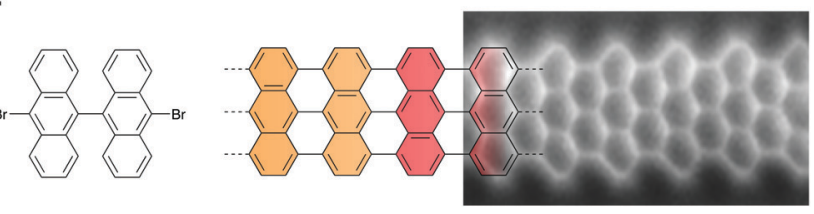

Meta
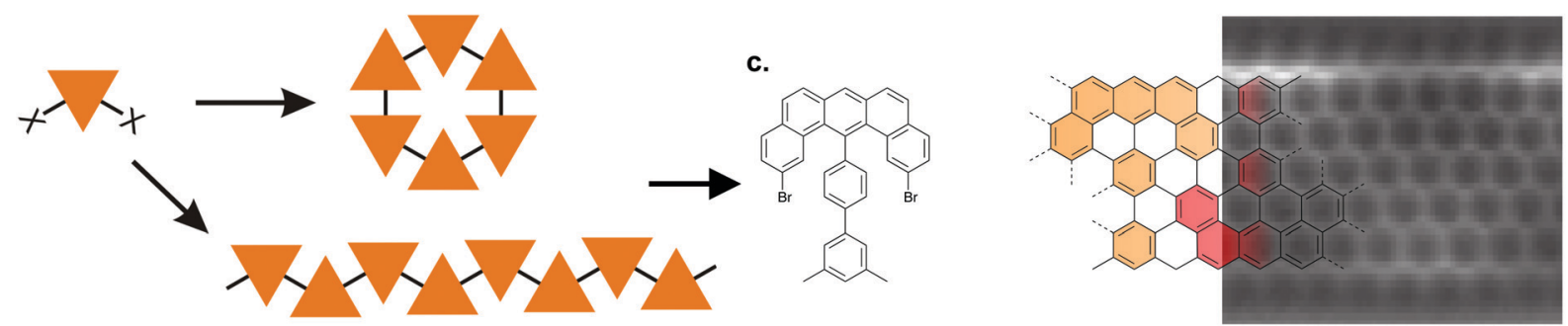

Fig. 2 Precursor design determines the final product. (a) Schematic overview of the Ullmann coupling reaction in dependence of precursor design. $X$ represents a halogen atom. Adapted with permission from ref. 49. Copyright 2017, the Royal Society of Chemistry. (b) Synthesis of a GNR from a precursor with halogen substitutions in opposite positions leading to an AGNR. The example depicts the formation of a 7-AGNR from dibromo-bianthryl together with a high-resolution non-contact (nc) atomic force microscopy (AFM) image recorded with a CO-functionalized tip. Adapted with permission from ref. 179. Copyright 2013, Springer Nature. (c) Synthesis of a GNR from a precursor having the halogens in the 'meta' position together with a highresolution nc-AFM image recorded with a $\mathrm{CO}$-functionalized tip. Steric repulsion prevents the formation of a cyclic nanographene. Adapted with permission from ref. 58. Copyright 2016, Springer Nature. 
hindrance favours/excludes the formation of certain bonds (e.g. 11). Using such a design strategy, atomically precise 9-, 13- and 17-AGNRs have been successfully synthesized. ${ }^{56,57}$ In addition, steric hindrance has also been identified as a useful concept for the synthesis of cGNRs and chGNRs.

Placing the halogen substitutions not on opposite ends of the precursor (e.g. 50 and 65) can lead to the formation of a polymer with a zigzag structure (Fig. 2, orange building blocks). However, special care must be taken - for example by incorporating elements of steric hindrance - to prevent the formation of a cyclic nanographene. Using such design considerations, ZGNRs $^{58}$ have been successfully synthesized (Fig. 2c), which are otherwise inaccessible as Ullmann-type coupling normally takes place along the armchair directions of graphene.

The use of prochiral precursors allows the fabrication of complex edge structures from relatively simple precursor molecules. In the case of homochiral coupling, the resulting GNR will be chiral (e.g. 55 and 57) whereas in the case of heterochiral coupling, the resulting GNR will be achiral (e.g. 42). However, $a$ priori it is difficult to determine which coupling orientation will be preferred, which complicates the rational design using prochiral precursors. ${ }^{59}$ It should be noted that while the above discussed precursor design considerations allow for precise control over the width and edge orientation of GNRs, the precise control over their length remains a challenge which has not been solved until now.

Role of the substrate. The substrate plays a crucial role in all three steps necessary for the formation of GNRs. Not only does it confine the building blocks to a two-dimensional plane, it also serves as the catalyst for dehalogenation. ${ }^{51,60}$ Because of their catalytic activity, coinage metal surfaces, such as $\mathrm{Au}(111)$, $\mathrm{Ag}(111)$ and $\mathrm{Cu}(111)$, are typically used. Nevertheless, Ullmann-type coupling also has been reported for molecules on graphene, ${ }^{61}$ hexagonal boron nitride ${ }^{61-63}$ and bulk insulators, ${ }^{64,65}$ further highlighting the versatility of this synthesis method. However, synthesis of GNRs on these non-metallic substrates remains challenging, as the adsorption energy on these surfaces is typically lower than that of metals. This, together with the higher temperature required for the on-surface synthesis which is due to their low catalytic activity, promotes desorption of molecules before any coupling reactions can occur. ${ }^{64,66}$

During the second step (i.e. the diffusion of the surface stabilized radical) the substrate once again plays a vital role. For long-range ordered structures, a relatively high precursor mobility (i.e. a low diffusion barrier) is desirable, whereas the precursor reactivity should be low. ${ }^{60}$ Note that the on-surface reactivity of the precursor also depends on the catalytic activity of the substrate. A theoretical study of Björk et al., ${ }^{52}$ using bromobenzene as a model molecule, showed that $\mathrm{Cu}(111)$ is the worst candidate of the three most commonly used (111)-oriented coinage metal surfaces in this respect since it has the highest diffusion barrier together with the largest catalytic activity. Interestingly, $\mathrm{Ag}(111)$ has a similar diffusion barrier as $\mathrm{Au}(111)$, but a lower catalytic activity making it the best surface for synthesizing highly ordered structures. In a combined experimental and theoretical study comparing $\mathrm{Ag}(111), \mathrm{Cu}(111)$ and $\mathrm{Au}(111)$, using a sixfold iodine substituted cyclic precursor, Bieri et $a .^{60}$ found similar results: the most well-ordered networks were formed on $\mathrm{Ag}(111)$, whereas the networks on $\mathrm{Cu}(111)$ were the least ordered. Nevertheless, $\mathrm{Au}(111)$ has remained the most widely used surface, also for GNR synthesis. When determining the right substrate for GNR synthesis another consideration has to be taken into account, namely the availability of metal adatoms originating from the metal surface. The surface-stabilized radicals left upon dehalogenation can - together with adatoms available from the metallic substrate - form metal-organic polymers which in some cases can $^{67,68}$ and in other cases cannot ${ }^{59,68}$ be converted to GNRs. The energy required to generate adatoms on either $\mathrm{Cu}(111)$ or $\operatorname{Ag}(111)$ is less than on $\mathrm{Au}(111)$, i.e. adatoms are more abundant on the former two surfaces and thus, it is more likely to obtain metal-organic (intermediate) structures. ${ }^{69}$

Commonly, the (111)-oriented coinage metal surfaces have been used. However, the (110)-oriented coinage metal surfaces offer an anisotropic atomic arrangement with a row-like structure which provides the opportunity to guide the growth of GNRs. It nevertheless has to be considered that the synthesis is sensitive to the surface orientation as well. Using 10,10'-dibromo-9,9'bianthracene (8) as a molecular precursor, a GNR could be readily formed on $\mathrm{Cu}(111),{ }^{70-73}$ but on the more reactive ${ }^{74}$ $\mathrm{Cu}(110)$ substrate no GNR could be formed as the precursor bonded too strongly to the substrate. ${ }^{75}$ On $\mathrm{Au}(110), 8$ can form a GNR, but owing to the lower mobility on this surface, the lengths of the formed GNRs were limited. ${ }^{76}$ A more successful strategy of aligning GNRs is to synthesize them on vicinal surfaces, such as $\mathrm{Au}(788)$ or $\mathrm{Au}(322) .{ }^{77-80}$ Because the terraces of these surfaces are $\mathrm{Au}(111)$ facets, results obtained on $\mathrm{Au}(111)$ can readily be replicated on $\mathrm{Au}(788)$, provided that the width of the GNR is not larger than the width of the terrace.

Lastly, the substrate material can influence how the precursors will couple to each other and that can even result in coupling schemes not intended for the designed precursor. For instance, on $\mathrm{Au}(111) 8$ forms an AGNR as indicated in Fig. $1 b^{37}$ whereas on $\mathrm{Cu}(111)$ it forms a chGNR with a combined armchair and zigzag edge (Fig. 3c) based on surface-assisted dehydrogenative coupling and not on Ullmann-type coupling. ${ }^{70-72}$

Role of the substituted halogen. The most prominent difference between the three commonly used halogens, $\mathrm{Br}$, I and $\mathrm{Cl}$, is the difference in energy required for dehalogenation. ${ }^{52,81}$ $\mathrm{C}-\mathrm{I}$ bonds require the least amount of energy for dissociation, whereas $\mathrm{C}-\mathrm{Cl}$ bonds require the most. Thus, using iodine can be advantageous for a low-temperature synthesis of the polymer intermediate as well as to increase the gap in temperature required for dehalogenation and cyclodehydrogenation, which leads to fewer side-reactions. ${ }^{49,82}$ Indeed, C-I bonds can be cleaved upon deposition of the precursors onto a substrate held at room temperature and the subsequent formation of $\mathrm{C}-\mathrm{C}$ bonds can then even proceed. ${ }^{83,84}$ That means Ullmann-type coupling takes place at room temperature without external thermal input. In a comparative study using both bromine and iodine substituted precursors (11 and the iodinated analogue), the iodinated precursor was found to dehalogenate earlier (at lower temperature) and produce even longer ribbons compared 
a. $\mathrm{Br}$

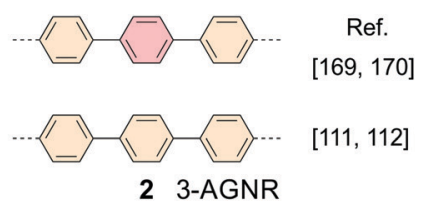

(Br)

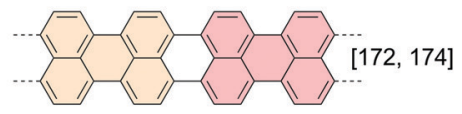

5

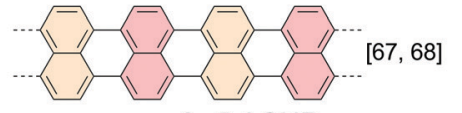

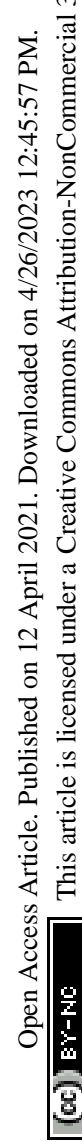

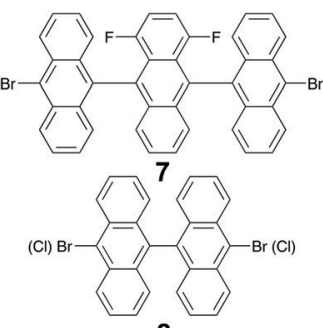

8
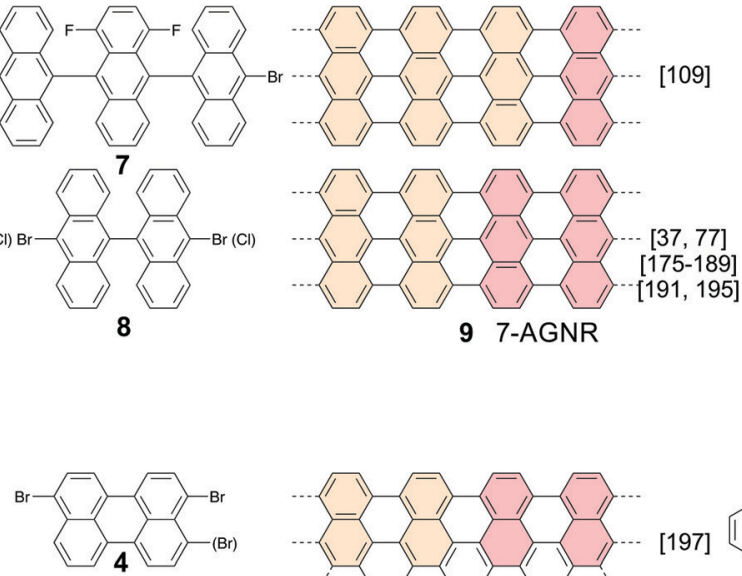

क्या

3

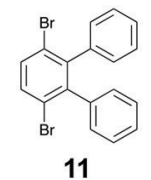

11

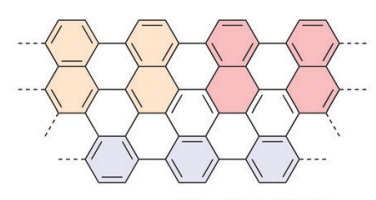

10 8-AGNR

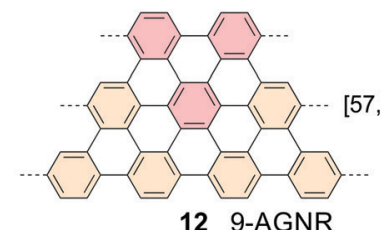

12 9-AGNR

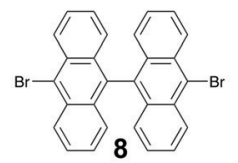

(1)

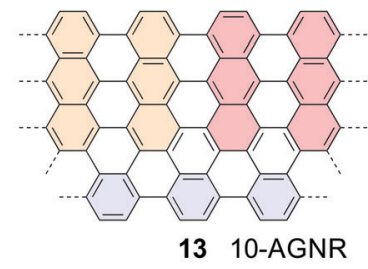

[197]

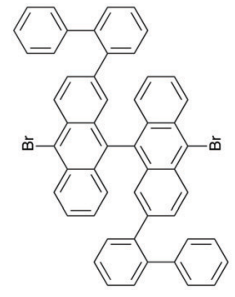

14

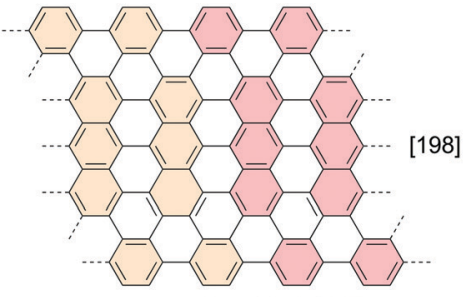

15 13-AGNR

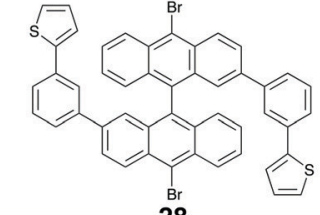

28
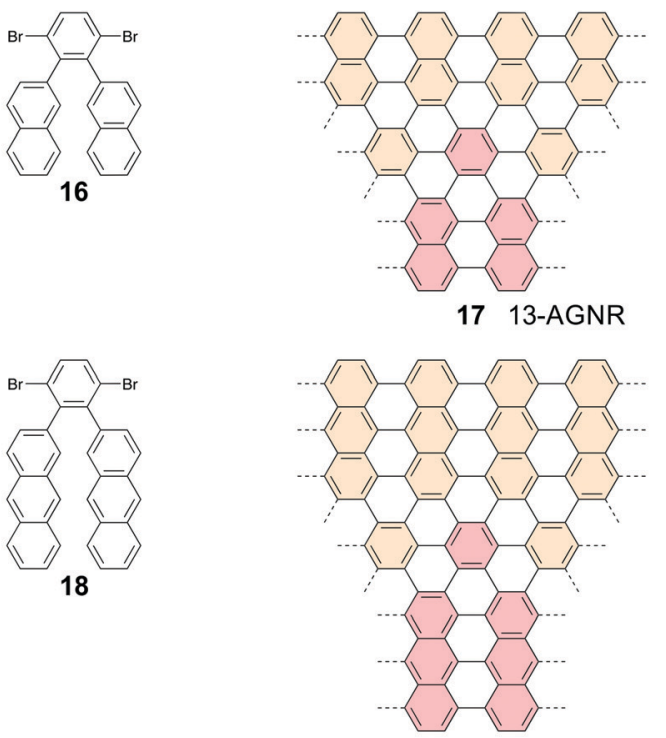

19 17-AGNR

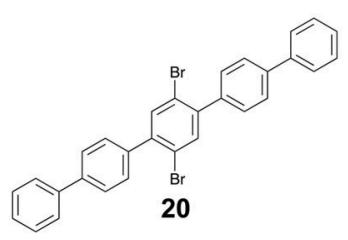

Ref.

[56]

[56]

[171]
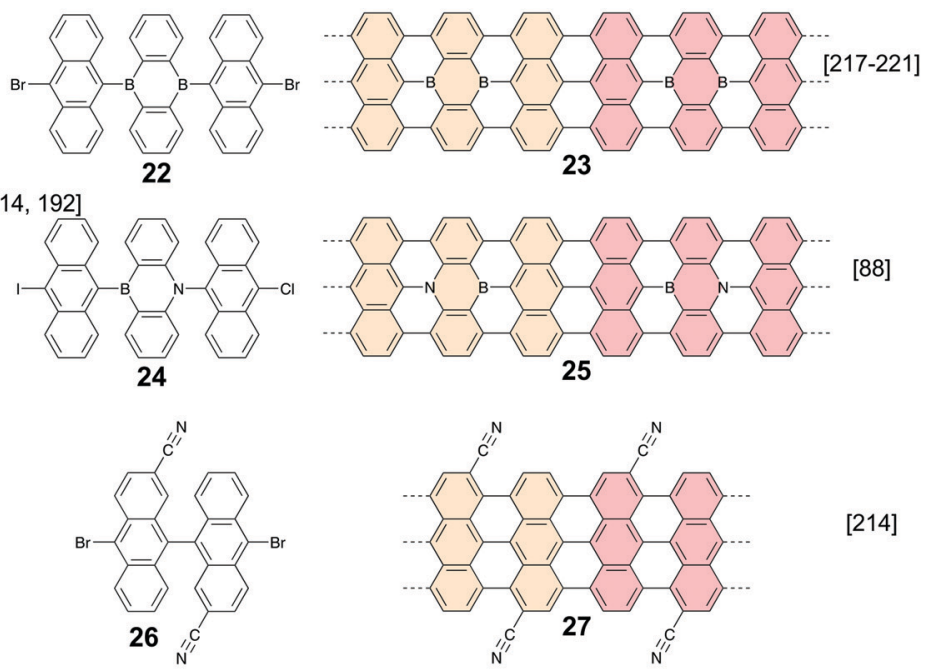

[214]

[215] 

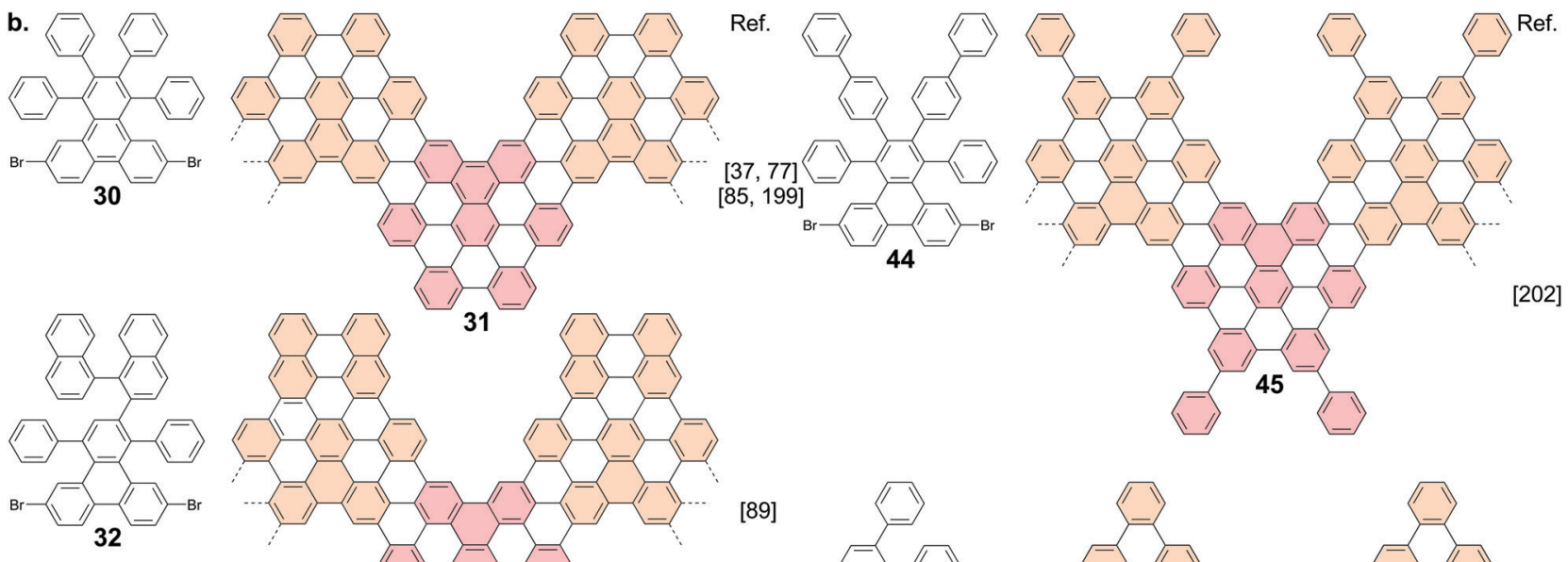

[89]
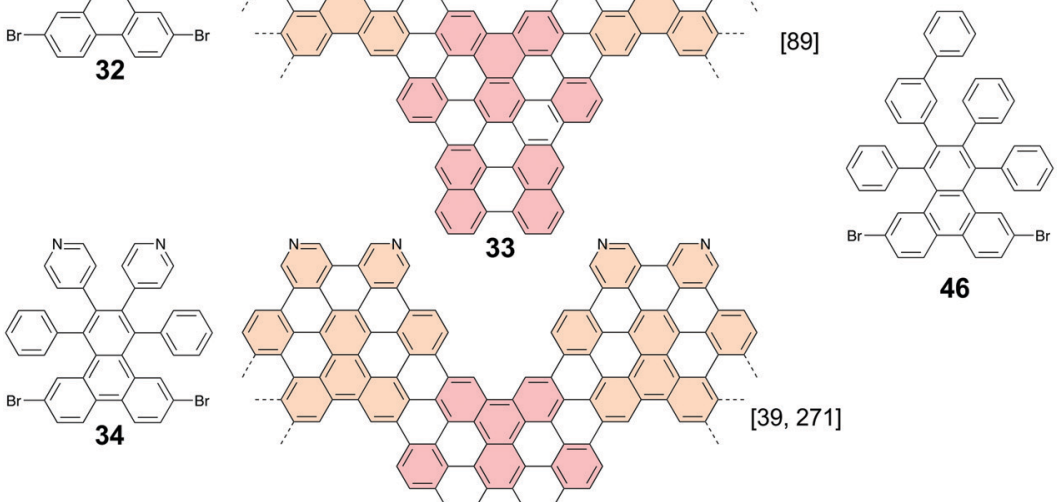

46
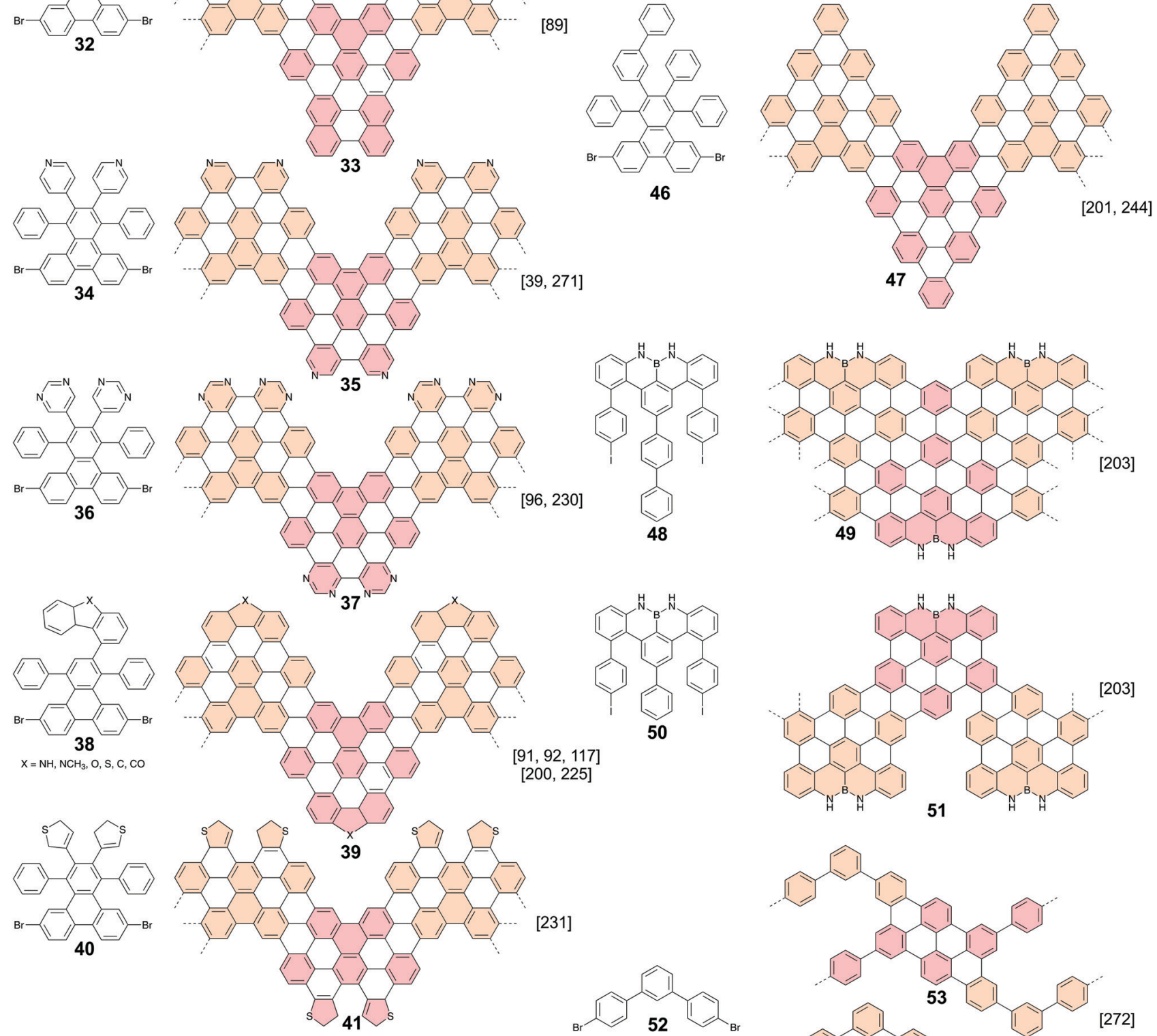

[231]

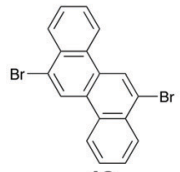

42

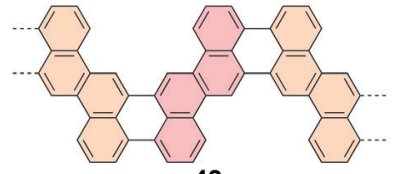

43

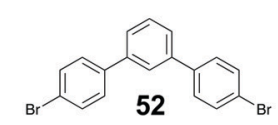

[59]
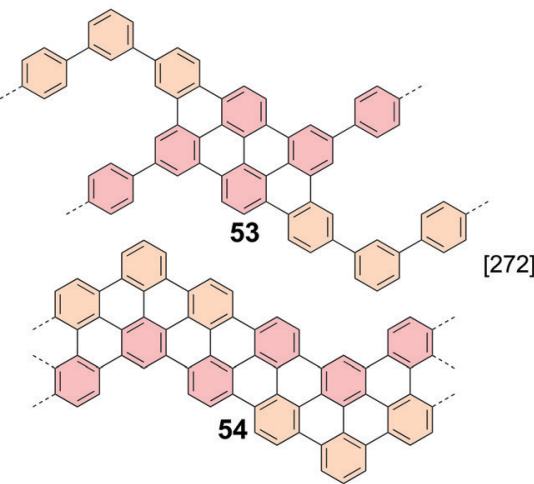


\begin{abstract}
c.
\end{abstract}<smiles>Cc1c([Hg])c(-c2ccccc2)c(-c2c3ccccc3c([Hg])c3ccccc23)c2ccccc12</smiles>

(1)

$\mathrm{N}^{\mathrm{Br}}$

55<smiles>CC(C)=C(C)c1c(Br)ccc2c(-c3ccccc3)c(Br)ccc12</smiles>

57

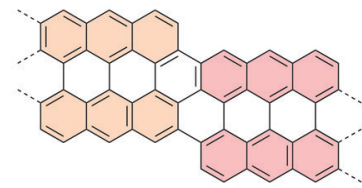

$56(3,1)$ ch GNR

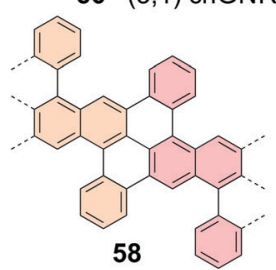

(1)

59

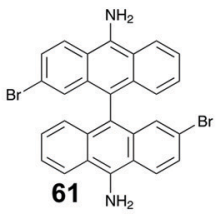

$61 \mathrm{NH}_{2}$

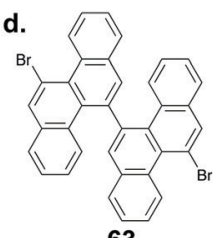

63

e.<smiles></smiles>

65

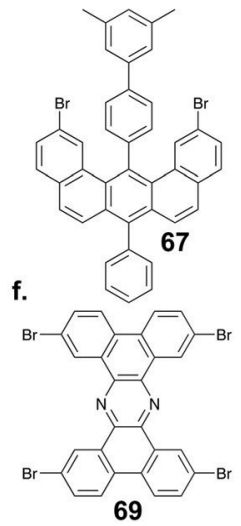

$\mathrm{NH}_{2}$

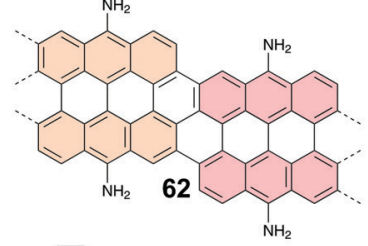

$\mathrm{NH}_{2}$

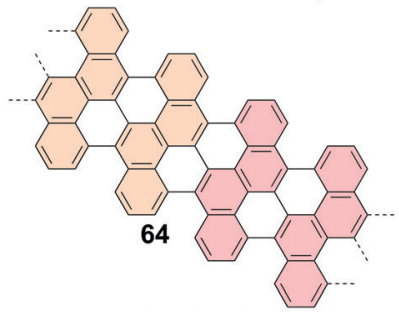

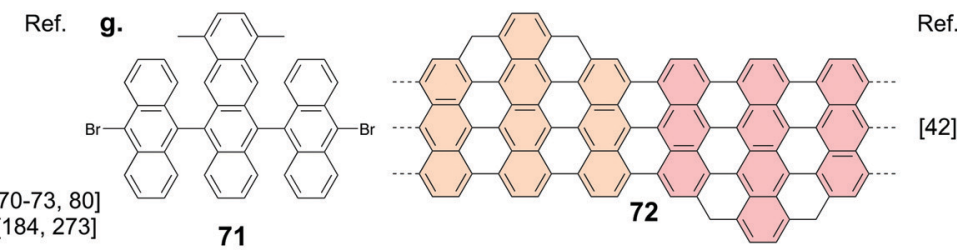

Ref.

[204]
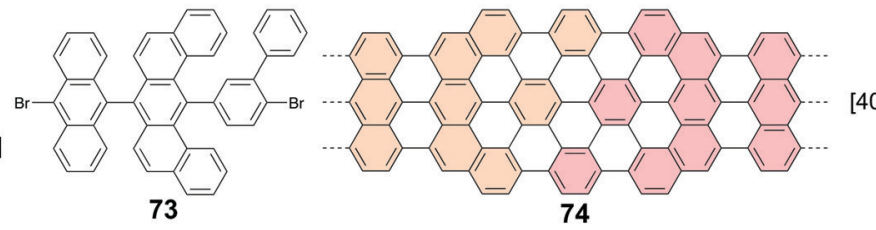

[40]

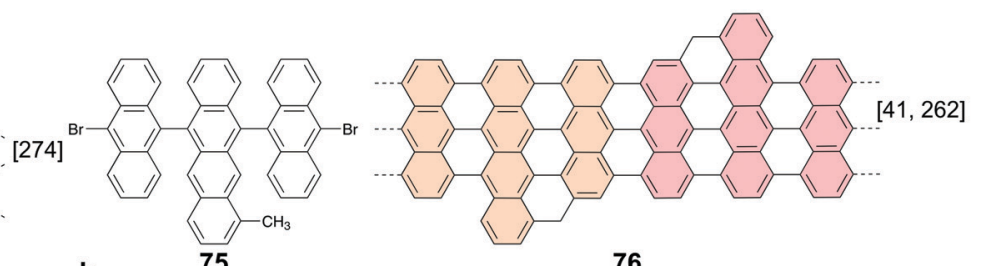

[216]

h.
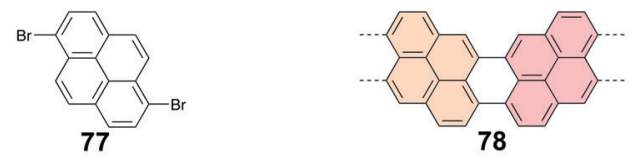

[213]

[275]
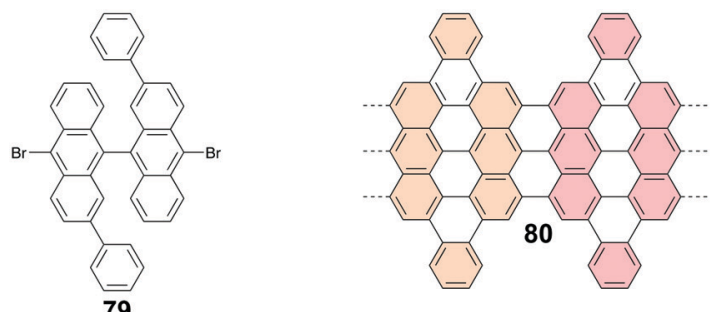

[116, 264]

[58]

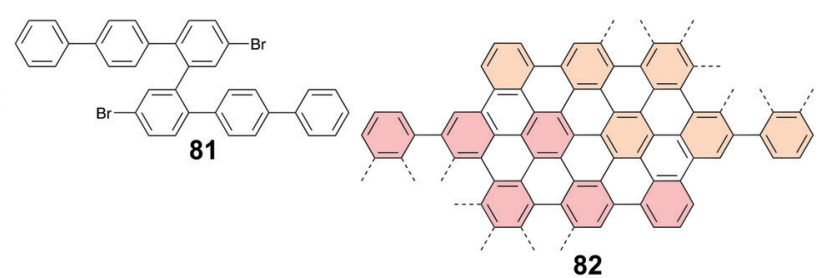

[276]

[210]

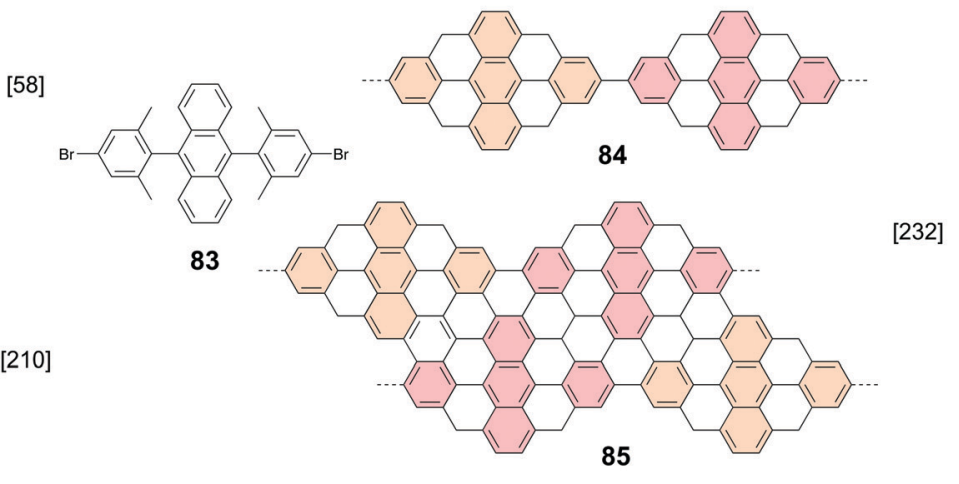

Fig. 3 Register of precursors used so far for atomically precise GNR formation together with the resulting GNR structure. (a) Armchair GNRs, excluding ones resulting from the lateral fusion of ribbons. (b) Chevron-like GNRs. (c) Chiral GNRs. (d) Cove-edged GNRs, (e) zigzag GNRs. (f) Porous GNR. (g) GNRs with embedded topological states. (h) GNRs with other edge topology. 
to the brominated precursor. This was ascribed to the reduced overlap between the polymerization and cyclodehydrogenation step. ${ }^{82}$ Contrastingly, when using a larger precursor (30) no difference was found for the energy required for polymer formation from either iodinated or brominated precursors, ${ }^{85}$ although dehalogenation did occur at lower temperatures for the iodinated precursors. This was ascribed to the low mobility of the surface-stabilized radicals, making the ribbon length diffusion-limited. Thus, using iodine to lower the energy barrier for polymerization may only be a viable option for molecules having a relatively low diffusion barrier when dehalogenated on the substrate, which will often be true for smaller molecules. Interestingly, Jacobse et al. ${ }^{86}$ when using the chlorinated analogue of $\mathbf{8}$, found that the stronger $\mathrm{C}-\mathrm{Cl}$ bond caused the cyclodehydrogenation to occur before Ullmann-type coupling. Crucially, the cyclodehydrogenation planarized the precursor molecules and no subsequent Ullmann-type coupling could take place. This was ascribed to the steric hindrance caused by the hydrogen atoms at the peri positions, preventing Ullmann-type coupling. Thus, the chlorinated precursor could not form a 7-AGNR on $\mathrm{Au}(111)$, in contrast with the brominated one.

The dissimilar amount of energy required for dehalogenation can be harnessed for hierarchical polymerization processes, where dissimilar halogen species are split off in a controllable, consecutive fashion, thereby allowing the formation of complex nano-architectures. ${ }^{83,87,88}$ An instructive example is the work of Bronner et al., ${ }^{89}$ where a combination of iodinated and brominated precursors was used together with a 'linker' molecule, which had both an iodine and bromine substitution, to form GNR heterostructures with preferentially only a single junction per GNR. Without such hierarchical processes, the formation of GNR heterojunctions relies on stochastics and multiple junctions (of the same type) may form within a single ribbon. ${ }^{90-96}$

After the halogen bond has been cleaved, the split-off halogen can remain adsorbed on the surface. It has been suggested that the adsorbed halogens may hinder polymer growth, ${ }^{52,97}$ negatively affecting GNR quality. Typically the split-off halogens desorb upon cyclodehydrogenation as hydrogen halides, leaving pristine GNRs after the synthesis is completed. ${ }^{82,97,98}$ However, at that point the growth has already been negatively impacted. Adsorbed halogens can be removed by annealing, however this requires considerably higher temperatures than those required for the initial Ullmanntype coupling and is unfortunately also substrate dependent. ${ }^{52} \mathrm{~A}$ better option may be to dose $\mathrm{H}_{2},{ }^{97,99,100}$ atomic hydrogen ${ }^{101}$ or $\mathrm{Si}^{102}$ which promotes the low-temperature desorption of halogens. Employing such techniques may help growing longer polymers and eventually GNRs, although care must be taken to avoid premature radical passivation with, for instance, hydrogen. ${ }^{103}$

\section{Cyclodehydrogenation}

The final step in GNR synthesis is the conversion of the polymer intermediate into a GNR through surface-assisted cyclodehydrogenation (Fig. 1b). Through cyclodehydrogenation, intramolecular aryl-aryl coupling occurs upon release of atomic hydrogen from the aryl units. Thereby, extra $\mathrm{C}-\mathrm{C}$ bonds are formed and often a planarization of the molecule takes place.
Although the experimental use of this reaction is abundant, relatively little theoretical work has been carried out with respect to the reaction mechanism. ${ }^{93,104,105}$

Cyclodehydrogenation mechanism. An illustrative work on surface-assisted cyclodehydrogenation was carried out by Treier et al. ${ }^{104}$ who studied the transformation of a cyclic polyphenylene into a nanographene on $\mathrm{Cu}(111)$ using a combination of STM and $a b$ initio density functional theory (DFT) calculations. With this combination, they were able to identify the different steps of the reaction process. Both the increased strain within the molecule, due to the van der Waals interactions with the substrate, and the catalytic activity of the copper substrate were found to be important factors in the reaction. A similar study by Björk et al., ${ }^{105}$ who studied the formation of 7-AGNRs from 8 on $\mathrm{Au}(111)$ using DFT calculations, found that the energy barrier for cyclodehydrogenation of a position surrounded by other non-dehydrogenated positions was $1.1 \mathrm{eV}$, whereas the energy for cyclodehydrogenation on positions neighbouring an already dehydrogenated position was reduced to only $0.08 \mathrm{eV}$. This result thus suggests that cyclodehydrogenation is a cooperative process: it starts at one end of the ribbon and propagates to the other end. Subsequent experimental studies found that, in partially dehydrogenated ribbons, non-dehydrogenated positions within a ribbon preferentially line up in rows, corroborating this cooperative mechanism..$^{93,106}$ A similar avalanche mechanism was found earlier for the formation of PAHs through the intramolecular Scholl reaction. ${ }^{107,108}$

The hydrogen released during the cyclodehydrogenation step was found to end ribbon growth by passivating the radical termini of the ribbons. Thus, after cyclodehydrogenation took place the ribbon can no longer increase in length. ${ }^{103}$

The requirement for the surface to be sufficiently catalytically active for cyclodehydrogenation to occur was demonstrated by the work of Kolmer et al. ${ }^{65}$ While 8 can polymerize through Ullmann-type coupling on the $\mathrm{TiO}_{2}(011)-(2 \times 1)$ surface, it cannot undergo cyclodehydrogenation as it does on $\mathrm{Au}(111) .{ }^{37}$ In recent work it was found that by strategically using fluorine substitutions (7) one can form nanographenes and GNRs on $\mathrm{TiO}_{2}$ through $\mathrm{C}-\mathrm{F}$ bond activation, eliminating the need for a catalytically active substrate. ${ }^{109,110}$ This represents a milestone in the on-surface synthesis of GNRs, as growing GNRs directly on semiconducting or insulating surfaces foregoes the need for transfer when incorporating the ribbons in devices.

Lateral fusion. Although the exterior hydrogen atoms of the ribbons remain unaffected during the cyclodehydrogenation process, by annealing beyond the temperature required for cyclodehydrogenation, a lateral fusion of GNRs can be induced leading to wider ribbons. ${ }^{80,111-117}$ This method was first used by Huang et al. ${ }^{113}$ to form 14- and 21-AGNR on Ag(111) from 1. Later, using 4,4"-dibromoterphenyl (3), 3n-AGNRs were synthesized on $\mathrm{Au}(111) .{ }^{111,112}$ Another example is the lateral fusion of chevron-like ribbons which results in the formation of coveted, atomically precise nanoporous graphene. ${ }^{116,117}$ While the above described formation methods are useful for scientific characterization of ribbons that are otherwise complicated to synthesize, the synthesis through this pathway suffers from a 
lack of selectivity and thus, is not suitable for application processes requiring specific GNR widths. However, by using a stepped $\mathrm{Au}(322)$ surface Merino-Diez et al. ${ }^{80}$ were able to preferentially form 6-AGNRs from 3, alleviating this problem.

\section{Electronic properties of graphene nanoribbons}

As graphene is a gapless semimetal, it is unsuitable for applications for which a band gap is required, such as field effect and optoelectronic devices. As such, the principal interest in GNRs is their semiconducting behaviour caused by quantum confinement of the charge carriers. However, GNRs also host a plethora of rich electronic behaviours depending on their width and edge termination. For instance, AGNRs have width-dependent band gaps, ${ }^{6,29,118,119}$ whereas ZGNRs are expected to host spin-polarized edge states, ${ }^{29,119,120}$ making them promising candidates for spintronic applications. On the other hand, cGNRs, with meandering topology, are expected to be more suitable for thermoelectric and optoelectronic applications than their straight counterparts. ${ }^{121-127}$ Moreover, the excellent control over their structure provided through on-surface synthesis has been harnessed to create engineered topological states, which received increasing attention in both theoretical ${ }^{128-131}$ and experimental studies. ${ }^{40-42}$

Subsequently, we will discuss the fundamentals of the electronic properties of different GNRs, as well as the experimental characterization of them.

\section{Theoretical background}

Due to the finite width of GNRs, the electron momenta are quantized in the transverse ribbon direction. An illustrative and intuitive way to approximate the band structure of GNRs is to start with the well-known tight-binding band structure of graphene and to make cuts along the allowed momentum values (Fig. 4a). In this way, a 1D band structure is formed out of the obtained conic sections. This approach is analogous to that employed for carbon nanotubes and the results are similar, too. ${ }^{132}$ Based on this approach, AGNRs can be divided into three classes: $N=3 p, N=3 p+1$ and $N=3 p+2$ where $p$ is a natural number and $N$ denotes the number of dimer lines along the edge, i.e. the width of the GNR (Fig. 1a). The AGNRs belonging to the $3 p+2$ family are found to be semimetallic within this approximation, whereas the ribbons of the other two families are semiconducting. ${ }^{6,119,120}$ From LDA-DFT calculations, it has been found that all GNRs of finite width are semiconducting, including AGNRs belonging to the $3 p+2$ family (Fig. $4 \mathrm{~b}) .{ }^{29}$ The hydrogen
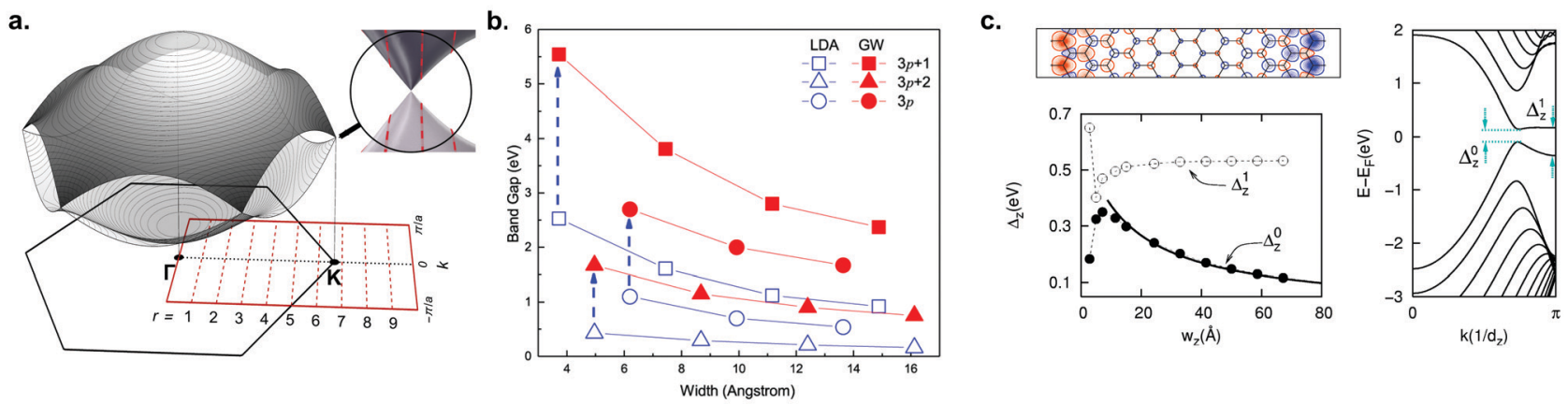

d.
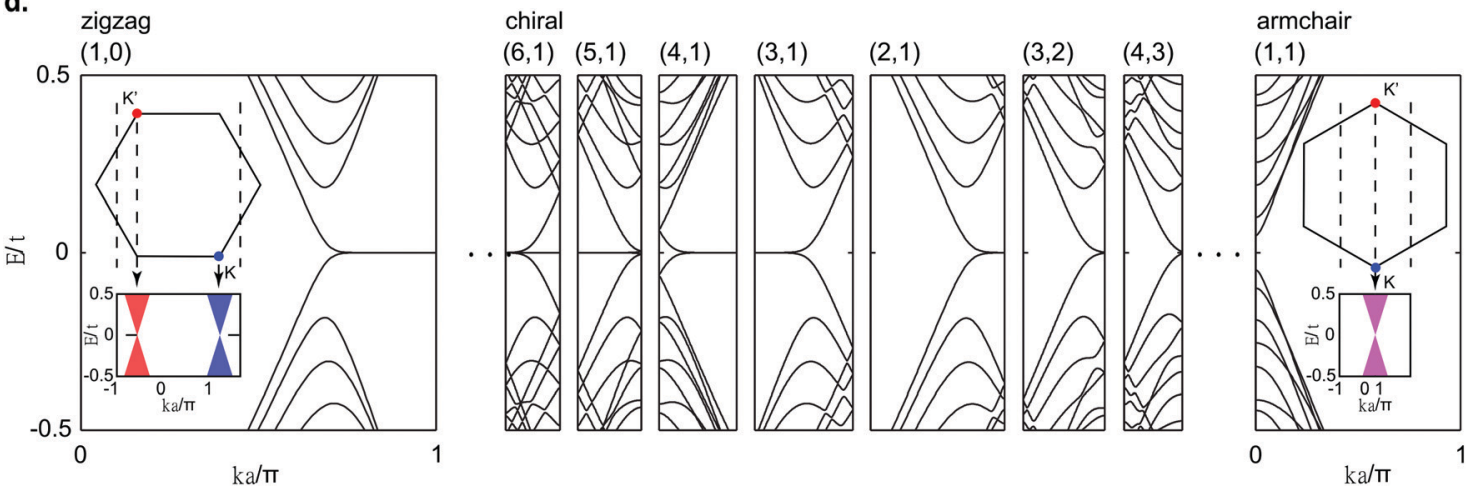

e.

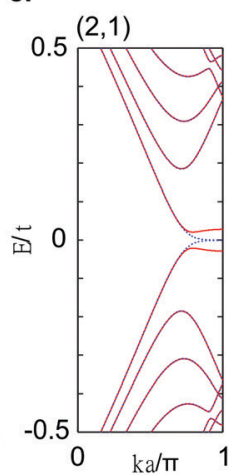

Fig. 4 Electronic properties of graphene nanoribbons. (a) Formation of the band structure of GNRs by selecting cuts along allowed $k$-values for a 9-AGNR. Reprinted with permission from ref. 57. Copyright 2017, American Chemical Society. (b) LDA-DFT calculations of AGNR band gaps and quasiparticle correction. Reprinted figure with permission from ref. 118. Copyright 2007 by the American Physical Society. (c) Electronic structure of ZGNRs from LSDA-DFT calculations, showing the spin-polarized edge state, formation of a band gap and band gap as a function of ribbon width. Reprinted figure with permission from ref. 29. Copyright 2006 by the American Physical Society. (d) Band structure of various zigzag, chiral and armchair GNRs from tight-binding calculations. Reprinted figure with permission from ref. 135. Copyright 2011 by the American Physical Society. (e) Mean-field Hubbard model calculations for a $(2,1)$ chiral GNR, showing the opening of a band gap. Reprinted figure with permission from ref. 135 . Copyright 2011 by the American Physical Society. 
passivation of the edges plays an important role in the opening of a band gap in the $3 p+2$ family, as the hydrogen atoms change the on site energy of the outer carbon atoms compared to the inner ones and consequently the bond lengths at the edges are altered. When this alteration is taken into account within the tight-binding model, the $3 p+2$ family is then also semiconducting. ${ }^{29}$

For ZGNRs, a flat band at the Fermi level, corresponding to an edge state that decays exponentially into the ribbon, arises from tight-binding calculations. ${ }^{6,120}$ The high DOS at the Fermi level originating from this flat band is expected to lead to a spin-polarization through electron-electron interactions, opening a band gap. ${ }^{119}$ Indeed, the Hubbard model and LSDA-DFT calculations predict a magnetic insulating ground state for ZGNRs. ${ }^{29,119,120,133}$ The spins are ferromagnetically ordered along one edge and antiferromagnetically coupled between edges (Fig. 4c). A band gap $\left(\Delta_{0}\right)$ arises that is related to antiferromagnetic correlation between the two edges, whereas $\Delta_{1}$ is related to the ferromagnetic correlation along the edges. ${ }^{134}$ As the ribbon gets wider $\Delta_{0}$ decreases, similar to AGNRs, whereas $\Delta_{1}$ remains constant. ${ }^{29,118}$

With on-surface synthesis, GNRs with arbitrary edge structure can be synthesized. Two of the most common ribbon types with alternating edge topologies are the cGNR and chGNR. A chGNR can be defined by three variables: its width and the numbers $m$ and $n$ of the graphene unit cell vectors $\mathrm{a}_{1}$ and $\mathrm{a}_{2}$, respectively, within one ribbon unit cell (Fig. 1a). Using this description, $(m, n)=(1,0)$ corresponds to a ZGNR, while $(1,1)$ corresponds to an AGNR. Without considering electron-electron interactions, chGNRs having a structure close to the one of ZGNRs, i.e. those with a large amount of zigzag edges within a unit cell, exhibit flat band dispersion similar to ZGNRs, whereas for chGNRs close to AGNRs the flat band is almost completely suppressed (Fig. 4d). ${ }^{6,135}$ When electron-electron interactions are taken into account, a band gap opens for all chGNRs (Fig. 4e). ${ }^{28,135-137}$ The band gap of chGNRs, similar to AGNRs, decreases with increasing width, but not monotonically. ${ }^{28,137-139}$

cGNRs are meandering GNRs with a periodic edge structure. The edge orientation can be exclusively armchair, zigzag or a combination of both. The electronic properties of these ribbons were extensively studied with the Hubbard model, DFT and the many-body $G W$ approach. $^{43,140,141}$ Interestingly, all ribbons with a zigzag edge are expected to exhibit an antiferromagnetic ground state, which should survive on $\mathrm{Au}(111)$ according to DFT calculations. ${ }^{141}$

Direct comparison of theory with experimental values is difficult. In a typical experiment used to probe the electronic properties of GNRs, e.g. (angle-resolved) photoelectron spectroscopy (ARPES) or scanning tunneling spectroscopy (STS), one does not directly measure the electron energy levels. Instead, charged excitations are measured resulting from the addition or removal of electrons during the measurements. Thus, care has to be taken when comparing experimental data to calculations based on non-interacting particles (e.g. tight-binding and DFT). In the specific case of GNRs, e-e interactions play an important role due to the weak screening and GNRs' (quasi-)1D nature. ${ }^{78,118,142-144}$ Using one-shot $G_{0} W_{0}$ calculations the quasiparticle gaps grow significantly when compared to single particle techniques like DFT (Fig. 4b). ${ }^{118}$ Lastly, the substrate plays a significant role through enhanced screening, lowering the quasiparticle bandgap when compared to an isolated ribbon. By combining $G_{0} W_{0}$ together with a semiclassical image-charge model good agreement between experiment and theory has been found. $^{78,127,144,145}$

Charge transport in graphene nanoribbons. The charge transport in GNRs is one of the most important properties for potential usage of GNRs in applications. Ballistic transport over several microns has been experimentally observed in GNRs that were epitaxially grown on SiC sidewalls, as evidenced by the quantized conductance in these systems. ${ }^{26,27}$ The transport has been extensively studied at various levels of theory in tandem with the Landauer-Büttiker formalism for pristine GNRs, ${ }^{123,137,146-149}$ GNR heterostructures, ${ }^{123,150-152}$ doped GNRs ${ }^{123,153}$ and GNR devices. ${ }^{150-152}$ From these studies, it has become evident that charge transport within GNRs is sensitive to defects, ${ }^{149,154}$ heteroatom doping ${ }^{123}$ and to the cleanliness of the underlying substrate. ${ }^{123}$

The possibility of quasiparticle-mediated charge transport notably through polarons - has been extensively studied on the tight-binding level of theory. ${ }^{155-165}$ It was found that stable polarons might form in narrow $(N \leq 8)$ AGNRs. ${ }^{157}$ In addition, interactions of these polarons with defects have been investigated and it was found that while some defects fully transmit polarons, others can reflect them. ${ }^{158,164}$

For implementing GNRs in devices, not only the intraribbon transport is important but also the inter-ribbon transport (ribbon-ribbon hopping), since the length of the channel will typically be larger than the length of a single GNR. Richter et al. ${ }^{166}$ employed a model previously successfully applied to organic semiconducting thin films ${ }^{167,168}$ to correctly describe the behaviour of GNR thin film devices. For such devices they found that the limiting factor for charge transport is the interribbon hopping. ${ }^{79,166}$

\section{Experimental characterization of GNRs}

Armchair graphene nanoribbons. The opening of a band gap, together with their experimental availability due to their facile synthesis, makes AGNRs the most researched class of GNRs. AGNRs with varying widths, ranging from 3- to 21-AGNRs have been so far synthesized and characterized experimentally.

The narrowest possible GNR is the 3-AGNR, which is a poly( $p$-phenylene) (PPP) wire. Unlike wider GNRs, PPP wires are non-planar and do not feature the same delocalized $\pi$-system as wider AGNRs. Up to now, PPP wires have been synthesized on $\mathrm{Cu}(110), \mathrm{Au}(111)$ and vicinal $\mathrm{Au}(322){ }^{80,111,112,169,170}$ The band gap of these wires adsorbed on $\mathrm{Au}(111)$ was reported to be between 3.05 and $3.23 \mathrm{eV}^{80,112}$ However, the principle interest in PPP wires has been in the creation of $3 p$ (medium- 4 ) GNRs through lateral fusion (Fig. 5a). In this way, 6-, 9-, 12- and 15-AGNRs were obtained. Their characterization with STS $^{112}$ yielded values for the band gaps of $1.69 \mathrm{eV}, 1.35 \mathrm{eV}, 1.13 \mathrm{eV}$ and $1.03 \mathrm{eV}$, respectively. Note that, although the 9- and 15-AGNRs were synthesized using 

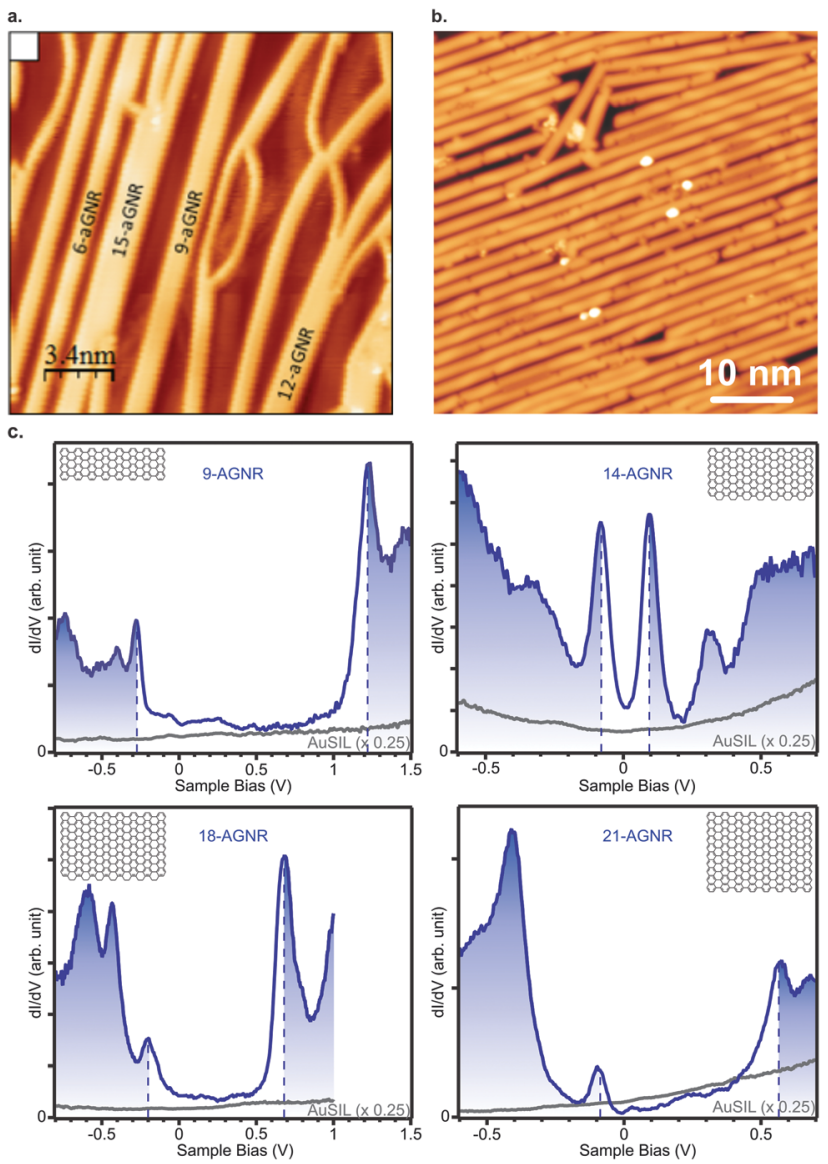

Fig. 5 (a) Lateral fusion of poly( $p$-phenylene) wires leads to formation of 3p-AGNRs. Reprinted with permission from ref. 112. Copyright 2017, American Chemical Society. Provided under an ACS AuthorChoice License, requests for further permissions should be directed to the American Chemical Society. (b) Long 9-AGNRs formed on Au(111) from an iodine functionalized precursor. Reprinted with permission from ref. 82. Copyright 2017, American Chemical Society. (c) STS spectra for 9-, 14-, 18- and 21-AGNRs on Si/Au(111) from which the band gap can be determined. Reprinted with permission from ref. 114. Copyright 2017, American Chemical Society.

other precursors as well, ${ }^{57,171}$ at the time of writing the 6- and 12-AGNR remain inaccessible through other means.

The 5-AGNR is the narrowest in the small- $\Delta$ family of GNRs and is well-researched. The reported values for the band gap of the 5-AGNR differ significantly. The first time it was fabricated, by Zhang et al., ${ }^{67}$ its band gap was identified as $2.8 \mathrm{eV}$ with STS. This is significantly larger than the value of $1.32 \mathrm{eV}$ which was obtained for isolated 5-AGNRs with many-body $G W$ calculations. This discrepancy was explained by the hybridization of the GNRs with the $\mathrm{Au}(111)$ surface. Later, Kimouche et al. ${ }^{172}$ carried out a systematic study of the band gap versus ribbon length and identified additional orbitals which appeared less prominent in STS experiments and found a band gap of $100 \mathrm{meV}$ for 5-AGNR on $\mathrm{Au}(111)$. Similar results were found by Zdetsis and Economou ${ }^{173}$ who found with time-dependent DFT that the band gap of long $(>10 \mathrm{~nm}$ ) ribbons is close to $100 \mathrm{meV}$. Recently, Lawrence et $a{ }^{174}$ reported a band gap of $0.85 \mathrm{eV}$ for the 5-AGNR on $\mathrm{Au}(111)$ and additionally identified two topological end-states (see also the theoretical work of Cao et al. $^{129}$ ). The end-states only appear when the ribbon is sufficiently long ( $>16$ unit cells, on shorter ribbons the end-states hybridize into a bonding and anti-bonding orbital and are delocalized over the ribbon). Since Kimouche et al. ${ }^{172}$ only report data for ribbons up to a length of 14 unit cells, it is feasible that what they identified as the valence and conduction band are the end-states identified by Lawrence et al. ${ }^{174} \mathrm{On} \mathrm{Ag}(111)$, the band gap of the 5-AGNR was reported to be $1.3 \mathrm{eV} .^{68}$ Note that the surface also has some influence on the band gap of adsorbed GNRs. ${ }^{144,145}$

The first-ever synthesized and most well-researched GNR is the 7-AGNR. In addition, it is the narrowest GNR in the large- $\Delta$ family that has been synthesized so far. 7-AGNRs were synthesized from 8 on $\mathrm{Au}(111),{ }^{37} \mathrm{Ag}(111),{ }^{113}$ and $\mathrm{TbAu}_{2} / \mathrm{Au}(111)^{175}$ and recently on $\mathrm{TiO}_{2}(011)-(2 \times 1)^{109}$ from 7 . Its properties have been extensively studied with a myriad of techniques: with STS, ${ }^{78,109,113,114,175-182}$ Fourier transformed (FT-)STS, ${ }^{180,182}$ (angle-resolved) photoelectron spectroscopy (ARPES), ${ }^{77,78}$ X-ray photoelectron spectroscopy (XPS), ${ }^{183,184}$ Raman spectroscopy, ${ }^{37,113,185,186}$ UV-vis spectroscopy, ${ }^{185}$ two-probe conductance measurements, ${ }^{73,177,178}$ high-resolution electron energy loss spectroscopy (HREELS), ${ }^{187}$ (angle-resolved) two-photon photoelectron spectroscopy, ${ }^{187,188}$ inverse photoemission spectroscopy (IPE), ${ }^{77}$ reflection difference spectroscopy (RDS) ${ }^{189}$ and STM induced light emission experiments. ${ }^{177}$ The band gap of 7-AGNRs measured with STS range from $2.3 \mathrm{eV}$ to $2.7 \mathrm{eV}^{78,109,114,175,176,178,180,182}$ which is in good agreement with theory. Similarly, with HREELS, 2PPE and IPE the band gap of 7-AGNRs was found to be in the range of 2.6 to $2.8 \mathrm{eV} .^{77,187,188}$ Note that the band gap depends somewhat on both the end termination of the ribbons $\left(\mathrm{CH}\right.$ or $\left.\mathrm{CH}_{2}\right)$ and their length, especially for short $(<8 \mathrm{~nm})$ ribbons. ${ }^{103,176}$ With RDS, the optical gap of aligned 7-AGNRs was determined by Denk et al. ${ }^{189}$ to be $2.1 \mathrm{eV}$. For the case of optical excitation, the gap is reduced compared to $G W$ calculations by the exciton binding energy whereas the surface plays a less dominant role than in the case of charged excitations (e.g. $\mathrm{d} I / \mathrm{d} V$ point spectroscopy experiments). ${ }^{190}$

By aligning 7-AGNRs with the help of a vicinal substrate, ${ }^{77,78}$ the band structure of the occupied states can be examined. With ARPES the effective mass was determined to be $m_{\mathrm{VB}}=$ $0.21-0.23 m_{\mathrm{e}}^{78,191,192}$ and $m_{\mathrm{VB}}=1.07 m_{\mathrm{e}}{ }^{77}$ and the charge carrier velocity to be $v=8.2 \times 10^{5} \mathrm{~m} \mathrm{~s}^{-1}$ (Fig. 6a). ${ }^{78}$ The effective masses found with AR-2PPE on randomly-oriented ribbons is $m_{\mathrm{VB}}=1.37 m_{\mathrm{e}}$ for the valence band and $m_{\mathrm{CB}}=1.35 m_{\mathrm{e}}$ for the conduction band. ${ }^{188}$ In FT-STS ${ }^{193,194}$ standing wave patterns caused by the diffraction of electrons are investigated by recording multiple STS point spectra along the length of a ribbon. In this way, the dispersion of individual GNRs can be obtained. Using this technique, effective masses of $m_{\mathrm{VB}}=0.41$ $m_{\mathrm{e}}$ and $m_{\mathrm{CB}}=0.40 m_{\mathrm{e}}$ were found for 7-AGNR on $\mathrm{Au}(111)^{182}$ and $m_{\mathrm{VB}}=0.32 m_{\mathrm{e}}$ and $m_{\mathrm{CB}}=0.35 m_{\mathrm{e}}$ on a decoupling $\mathrm{NaCl}$ layer (Fig. 6b). ${ }^{180}$ Obviously, the measured effective masses vary substantially. The origin of this effect was resolved by Senkovskiy et al. ${ }^{195}$ who found that the (first) valence band of aligned 7-AGNRs can only be observed with ARPES in a narrow range of emission angles. When the appropriate emission angle was chosen, the 
a.

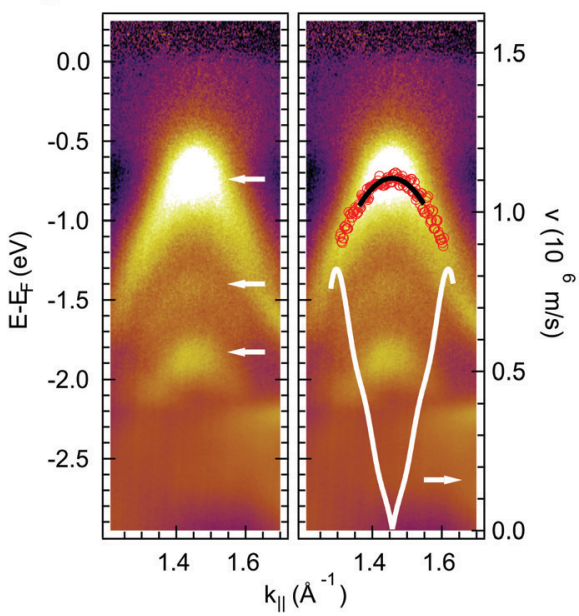

b.

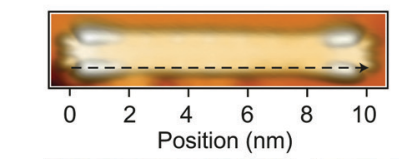

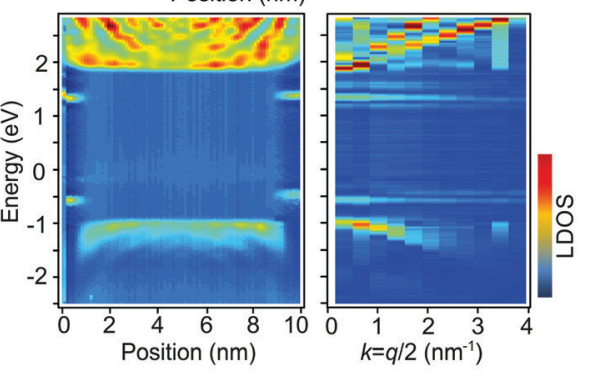

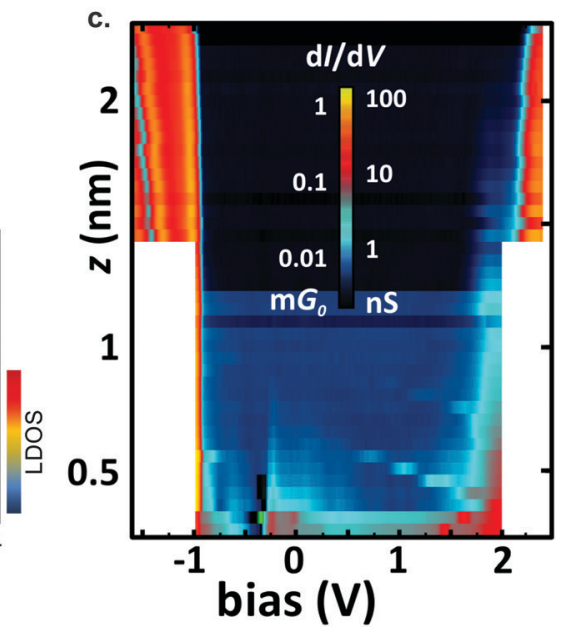

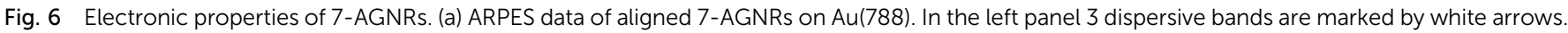

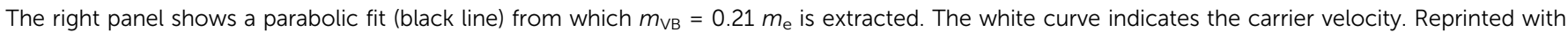

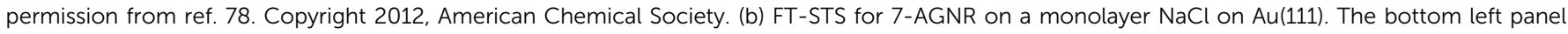

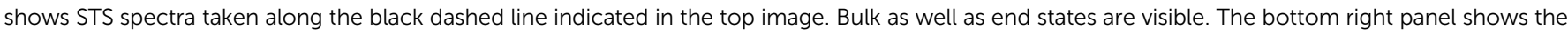

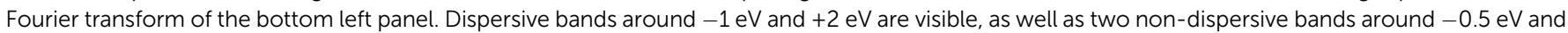

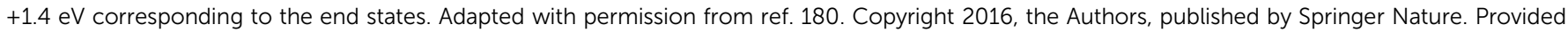

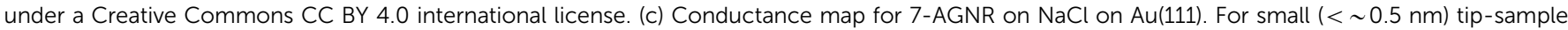

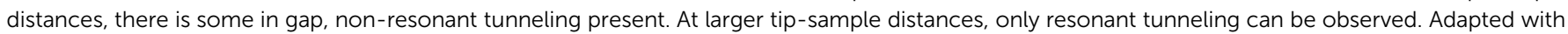
permission from ref. 73. Copyright 2018, American Chemical Society.

valence band was observed and an effective mass $m_{\mathrm{VB}}=0.4 m_{\mathrm{e}}$ was obtained in excellent agreement with the FT-STS results. The result of $m_{\mathrm{VB}} \approx 0.2 m_{\mathrm{e}}$ occurs when the second valence band is mistaken for the first one. The massive fermion behaviour of charge carriers in GNRs is a direct consequence of the opening of a band gap and GNRs with smaller band gap should thus have lower effective masses. ${ }^{78}$ This can be qualitatively understood from the 'cutting scheme' (Fig. 4a) described earlier: if the frontier cut is close to the Dirac point the resulting conic section will be 'sharper' and consequently the charge carriers have lower effective mass.

In addition to the delocalized valence and conduction electrons, 7-AGNRs (at least those obtained from 8) host localized end states at their termini which are associated with the local zigzag edge orientation. ${ }^{6,103,176-180}$ These end states are predicted to be spin-split, but for 7-AGNRs on Au(111) they were observed in STS experiments as a single peak, which is possibly degenerate due to hole-doping, close to the Fermi level. ${ }^{179}$ However, for 7-AGNR adsorbed on $\mathrm{NaCl}$, the zigzag end states are reported to split and a gap between them of $1.9 \mathrm{eV}$ is opened. In line with the localized nature of these states, they are found to be non-dispersive with FT-STS (Fig. 6b).

By picking up a 7-AGNR with an STM tip, a two-probe conductance experiment can be performed. ${ }^{196}$ As the tip-sample distance $L$ increases, the conductance is expected to decay as $\exp (-\beta(V) L)$ where $\beta(V)$ is the conductance decay parameter. ${ }^{73,196}$ When the applied bias is equal to a molecular energy level of the ribbon, $\beta$ decreases giving rise to resonant tunneling (Fig. 6c). ${ }^{73,178}$ The end states only provide a contribution to tunneling at small tip-sample distances due to their localized nature. Note that in this geometry (a part of) the ribbon is decoupled from the surface which leads to an alteration of their electronic properties compared to their adsorbed counterparts.

By lateral fusion of 7-AGNRs, both 14- and 21-AGNRs were obtained on $\mathrm{Au}(111)$ and $\mathrm{Ag}(111) .{ }^{113,114} \mathrm{~A}$ band gap of $0.2 \mathrm{eV}$ and $0.7 \mathrm{eV}$, respectively, was obtained from STS on $\mathrm{Si} / \mathrm{Au}(111)^{\mathbf{1 1 4}}$ (Fig. 5c). The larger gap for 21-AGNRs can be qualitatively understood since they belong to the medium- $\Delta$ family, whereas the narrower 14-AGNR belongs to the small- $\Delta$ family.

On the other hand, by lateral fusion of either 5-AGNRs or 7-AGNRs with a PPP wire, 8-AGNRs or 10-AGNRs were formed on $\mathrm{Au}(111) .{ }^{197}$ The band gap of these ribbons was measured to be $1.0 \mathrm{eV}$ and $2.0 \mathrm{eV}$, respectively. The value for the 8-AGNR presents a departure from the general trend that as the band gap decreases the wider the ribbons get, as the band gap for the smaller 5-AGNR, which belongs to the same (small- $\Delta$ ) family, was determined to be $0.85 \mathrm{eV} .^{174}$

The third and most well-researched AGNR in the medium- $\Delta$ family, the 9-AGNR, can be synthesized by clever use of steric hindrance from 11 on $\mathrm{Au}(111)$ (Fig. 5b). ${ }^{57}$ Its band gap is $1.4 \mathrm{eV}$ on $\mathrm{Au}(111)^{57}$ and $1.5 \mathrm{eV}$ on $\mathrm{Si} / \mathrm{Au}(111)$ (Fig. $\left.5 \mathrm{c}\right) .{ }^{114}$ With ARPES the effective mass $m_{\mathrm{VB}}=0.09 m_{\mathrm{e}}$ was found, whereas with FT-STS $m_{\mathrm{VB}}=0.12 m_{\mathrm{e}}$ and $m_{\mathrm{CB}}=0.11 m_{\mathrm{e}}$ were obtained. ${ }^{57}$ Compared to the 7-AGNR, this is in agreement with the prediction that GNRs with narrower band gaps have lower effective charge carrier masses. Two fused 9-AGNRs forming an 18-AGNR were reported to exhibit a band gap of $0.9 \mathrm{eV}$ on $\mathrm{Si} / \mathrm{Au}(111)$ (Fig. $5 \mathrm{c}$ ). ${ }^{114}$

The 13-AGNR, synthesized from 14, is the second-ever AGNR synthesized with atomic precision. Its band gap, determined with STS, is $1.4 \mathrm{eV}$ on $\mathrm{Au}(111)$ and it has a localized, in-gap end state originating from the zigzag edges at its terminus. ${ }^{198}$ Recently, the 13-AGNR was also synthesized from 16 by Yamaguchi et al. 
on $\mathrm{Au}(111) .{ }^{56}$ This leads to a ribbon with identical interior, but notably with a different edge orientation at the termini. They reported a similar band gap of $1.34 \mathrm{eV}$. However, they reported no end state for their ribbons. In addition, using FT-STS they found effective masses of $m_{\mathrm{CB}}=0.14 m_{\mathrm{e}}$ and $m_{\mathrm{VB}}=0.13 m_{\mathrm{e}}$.

Lastly, the widest GNR, which has been so far fabricated with atomic precision, is the 17-AGNR. It has a small band gap of $0.19 \mathrm{eV}$ on $\mathrm{Au}(111)$ and with FT-STS an effective mass $m_{\mathrm{CB}}=$ $m_{\mathrm{VB}}=0.06 m_{\mathrm{e}}$ was found. ${ }^{56}$

From the experimental characterization of AGNRs, the three predicted $\Delta$ families become apparent in accordance with theory. When selecting AGNRs for devices, a tradeoff has to be made between band gap and effective mass, where wider band gaps lead to larger effective carrier masses and therefore, lower electron mobility. Conversely, AGNRs with narrower band gaps will have higher electron mobilities.

Chevron graphene nanoribbons. Most cGNRs were synthesized using a 6,11-dibromo-1,2,3,4-tetraphenyltriphenylene (30) type precursor, which was first used by Cai et al. ${ }^{37}$ in 2010 . The band gap of $\mathbf{3 1}$ was reported to be in the range of 2.4 to $2.53 \mathrm{eV}^{92,199,200}$ as determined by STS, whereas with HREELS a larger gap value of $2.8 \mathrm{eV}$ was found. ${ }^{39}$ This is a substantially wider gap than the $1.03 \mathrm{eV}$ reported for the equally wide 15 -AGNR. ${ }^{112}$
In addition to 30, several doped variants were used to fabricate doped cGNRs (which will be treated in more detail below) as well as variants with additional edge functionalizations. From precursor 46, an extended cGNR (Fig. 7a) results which hosts an electronic band gap of $2.2 \mathrm{eV}^{201}$ On the other hand, the relatively similar GNR 45 has its band gap virtually unchanged compared to its parent GNR 31. The phenyl substitutions only affect the positions of the valence and conduction band which shift to lower energies. ${ }^{202}$ In addition, 45 can undergo lateral fusion to form various types of nanoporous graphene. ${ }^{202}$ Similarly, the GNR $39(\mathrm{X}=\mathrm{C})$ can undergo lateral fusion. In contrast, only a single type of atomically precise nanoporous graphene forms in this case (Fig. 7b). ${ }^{117}$

In addition, the synthesis of further cGNRs has been successfully achieved. For instance, two heterodoped GNRs were fabricated by $\mathrm{Fu}$ et $a l^{203}$ on $\mathrm{Au}(111)$ from the two similar precursors 48 and 50. Both GNRs are doped with nitrogen and boron. The electronic band gaps of GNRs 49 and $\mathbf{5 1}$ (Fig. 7c) were determined to be $1.5 \mathrm{eV}$ and $0.9 \mathrm{eV}$, respectively. These cGNRs are unique in the sense that they are the only ones so far with a partial zigzag edge topology.

By using prochiral precursors, cGNRs can be created from relatively simple precursors. For instance, the delicate interplay a.

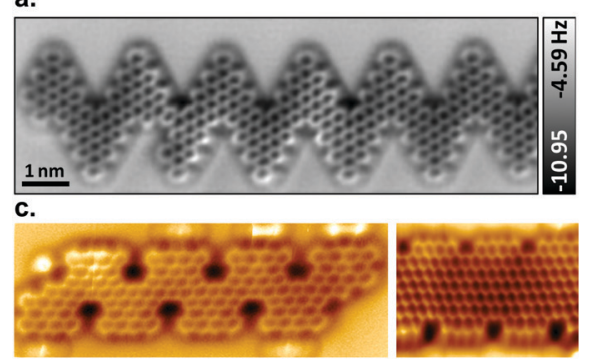

d.

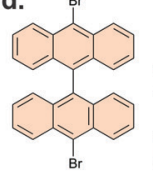

e.
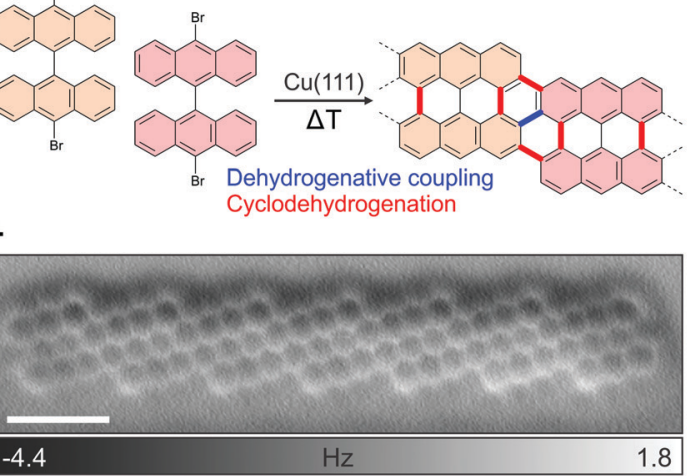

b.

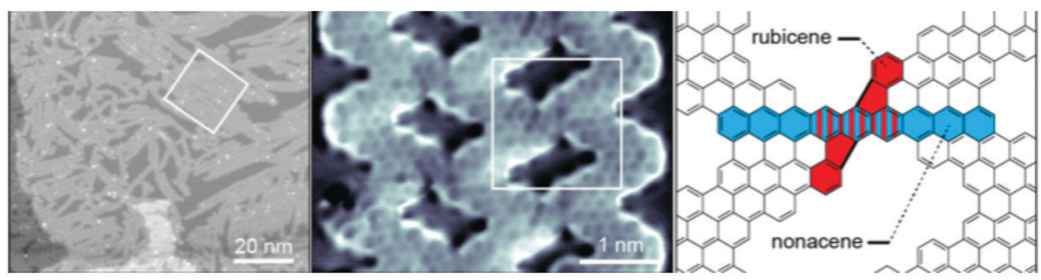

f.

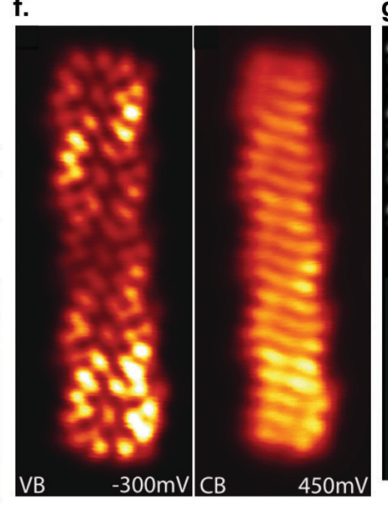

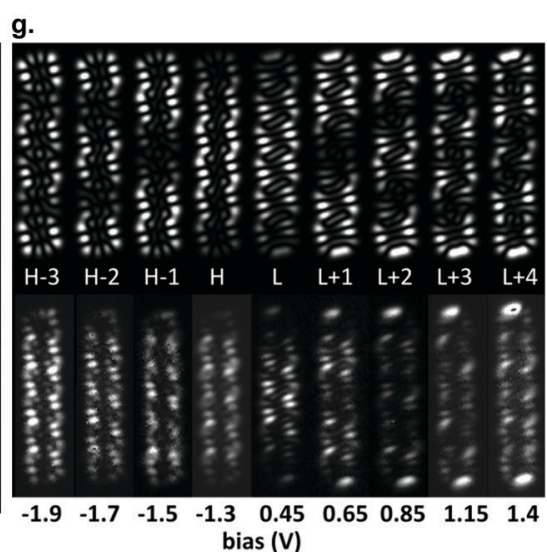

Fig. 7 (a) nc-AFM image of an edge extended cGNR synthesized from 46. Reproduced with permission from ref. 201. Copyright 2019, Wiley-VCH. (b) Nanoporous graphene from lateral fusion of cGNRs using $38(X=C)$ as a precursor. Reprinted with permission from ref. 117. Copyright 2020, American Chemical Society. (c) Nitrogen and boron doped chevron-like GNRs with partial zigzag edges fabricated from precursors $\mathbf{4 8}$ (right) and $\mathbf{5 0}$ (left). Reproduced with permission from ref. 203. Copyright 2020, the Authors, published by Wiley-VCH. Provided under a Creative Commons CC BY 4.0 international license. (d) Formation of (3,1)-chGNRs on Cu(111) based on the suggested synthesis of Sánchez-Sánchez et al. (ref. 72). (e) nc-AFM image of a $(3,1)$ chiral GNR formed from 9,9'-bianthracene (8 without halogen substitutions) on Cu(111). Reprinted with permission from ref. 208. Copyright 2017, American Chemical Society. (f) d//dV maps of the valence and conduction band of the (3,1) ch-GNR on Au(111). Reprinted with permission from ref. 209. Copyright 2017, American Chemical Society. (g) d//dV maps of $(3,1)$-chGNR on $\mathrm{NaCl}$. The top row reports the simulated data and the bottom row the experimental data. Reprinted with permission from ref. 73. Copyright 2018, American Chemical Society. 
of precursor and substrate led to the formation of the heterochiral GNR 42 on $\mathrm{Au}(111)$, a narrow cGNR. ${ }^{59}$

The theoretically predicted unique properties of cGNRs besides the determination of their band gaps - have not been experimentally verified thus far. For instance, due to the lack of cGNRs with pristine zigzag edges, the predicted anti-ferromagnetic ground state of those cGNRs could not be verified. ${ }^{43}$

Chiral graphene nanoribbons. Although chGNRs are predicted to have interesting electronic properties, such as width-dependent band gaps ${ }^{28}$ or spin-polarized edge state, ${ }^{6,135}$ only two (undoped) chGNRs are so far experimentally available: the $(3,1)$-chGNR $(56)^{71}$ and a benzo-fused $(2,1)$-chGNR $(58) .{ }^{204}$ In addition, current precursor design methods cannot easily be adapted to make either wider chGNRs or chGNRs with other edge orientations.

The formation of $(3,1)$-chGNRs from 8 on $\mathrm{Cu}(111)$ (Fig. $7 \mathrm{~d}$ ) was controversial with respect to the ribbon structure when the first reports appeared. ${ }^{71,205-207}$ With the help of nc-AFM imaging, the formation of a $(3,1)$-chGNR could be confirmed. ${ }^{72}$ It should be noted that 8 forms a 7-AGNR on $\mathrm{Au}(111)$. The formation of $(3,1)$ chGNRs from halogen-functionalized bianthryl precursors presents an exception: the formation is not based on Ullmann coupling but rather on a surface-assisted dehydrogenative coupling (blue bond in Fig. 7d). ${ }^{70-72,184,208}$ Indeed, 9,9'-bianthracene, without any halogen substitutions, forms a (3,1)-chGNR on $\mathrm{Cu}(111)$ as well (Fig. 7e). ${ }^{72,208}$

With STS, the band gap of $(3,1)$-chGNRs on $\mathrm{Au}(111)$ was determined to be $0.67 \mathrm{eV}$ (to form (3,1)-chGNRs on Au(111) 55 must be used as a precursor). ${ }^{209}$ The spatial distribution of the valence and conduction bands is shown in Fig. 7f. The charge carriers have an effective mass of $m_{\mathrm{VB}}=0.34 m_{\mathrm{e}}$ and $m_{\mathrm{VB}}=$ $0.36 m_{\mathrm{e}}$ obtained with FT-STS and ARPES, respectively, in good agreement with DFT calculations. ${ }^{209}$ Comparing this to the 7-AGNR, which has a comparable width, the effective masses are similar (the effective mass for the 7-AGNR is $m_{\mathrm{VB}}=0.41 m_{\mathrm{e}}$ and $m_{\mathrm{VB}}=0.4 m_{\mathrm{e}}$ obtained with FT-STS ${ }^{182}$ and ARPES ${ }^{195}$ respectively). However, the band gap of the 7-AGNR is much wider, approximately $2.4 \mathrm{eV}{ }^{182}$ Thus, the trend that in the case of AGNRs narrow band gaps lead to lower effective masses does not hold for GNRs with more complicated edge structures. By decoupling the $(3,1)$-chGNR from the metallic substrate with a layer of $\mathrm{NaCl}$, the electronic properties of the ribbon changed dramatically. The band gap widened to $1.8 \mathrm{eV}$ and the electronic orbitals became more localized on the edges of the ribbon (Fig. 7d). ${ }^{73}$ Changes of this magnitude were not observed for AGNRs (compare for instance a 7-AGNR, which has a band gap of $\sim 2.4 \mathrm{eV}$ on $\mathrm{Au}(111)$ and $\sim 2.9 \mathrm{eV}$ on $\mathrm{NaCl}) .{ }^{180}$ A possible origin may lie within the stronger interaction of zigzag edges with the metal substrate (as compared with armchair edges), which was also observed for the 6-ZGNR. ${ }^{58}$

Although the predicted spin-split edge states have been observed for chGNRs originating from the chemical unzipping of CNTs, ${ }^{19}$ such results have not yet been found for bottom-up synthesized chGNRs.

Zigzag graphene nanoribbons. ZGNRs are of particular interest for spintronics applications due to their theoretically predicted spin-polarized edge states. , $29,119,120,134,148$ Their experimental realization is however challenging. This is due to the fact that Ullmann-type coupling typically occurs along an armchair direction of graphene. So far, only the 6-ZGNR has been synthesized. ${ }^{58}$ By decoupling the ribbons from the metallic substrate with $\mathrm{NaCl}$, a band gap of $\Delta_{0}=1.5 \mathrm{eV}$ and an additional energy gap $\Delta_{1}=1.9 \mathrm{eV}$ were found. It will be interesting to see more investigations of ZGNRs, also in relation to their predicted spin properties, as they are currently - from an experimental point of view - poorly investigated.

Other ribbons. In addition to armchair, zigzag, chevron and chiral GNRs, GNRs with a more complex edge structure can be fabricated with on-surface synthesis. For instance, N-doped porous GNRs (70), i.e. GNRs having periodic vacancies, were synthesized recently (Fig. 8a). ${ }^{210}$ These present an interesting new class of ribbons, as the atoms in each of graphene's sublattices possess unique spin properties. ${ }^{211,212}$ Therefore, deliberately placing (periodic) voids in GNRs may be a way to introduce tunable spin properties.

Another recent advancement in the field attracting considerable attention is the formation of engineered topological states in GNRs. ${ }^{40-42}$ The ribbons created up to now (Fig. 3f) have focused on what can be interpreted as periodically repeating 7-9 AGNR heterojunctions. Depending on the boundary region, the 7- and 9-AGNR sections may belong to inequivalent topological classes leading to the formation of topological interface states. ${ }^{129}$ This can be harnessed to introduce topologically derived in-gap bands (Fig. 8b). ${ }^{40-42}$

GNRs of unconventional shape may also be used to further tune the electronic properties of GNRs. For instance, pyrene GNRs (78) have a remarkably narrow band gap of $E_{\mathrm{g}}=0.12 \mathrm{eV}$ (Fig. 8c) and the lowest effective charge carrier masses $m_{\mathrm{VB}}=m_{\mathrm{CB}}=0.02 m_{\mathrm{e}}$ reported up to date. ${ }^{213}$ This is an even narrower gap than the one reported for the 5- and 8-AGNRs which belong to the small- $\Delta$ family.

Doping. GNR doping can easily be introduced by adding heteroatoms, e.g. nitrogen or boron, to the precursor molecule. This gives rise to atomically precise doping. The usefulness of doping in this way is two-fold: on the one hand, the electronic properties, such as the band gap, can be tuned. On the other hand, the band alignment can be changed.

Edge doping (i.e. the dopant atoms are situated on the ribbon edges, such as in structures 35 and 37) with nitrogen atoms almost does not change the band gap compared to undoped ribbons, but it causes a downshift of $0.1 \mathrm{eV}$ per nitrogen atom present in the precursor molecule of both the valence and conduction band (Fig. 9a). ${ }^{39,96}$ On the other hand, cyano-functionalization of 7-AGNR (27) was shown to narrow the band gap by 0.1 to $0.2 \mathrm{eV}$ due to the extension of the $\pi$-system with an extra carbon atom. Since some CN moieties split off during the reaction, regions with both one and two cyano groups per unit cell were found in the ribbons. A rigid downshift of 0.2 to $0.3 \mathrm{eV}$ per $\mathrm{CN}$ group present in the precursor was observed, ${ }^{214}$ much more than the shift per nitrogen atom for edge doping. Thus, not only the heteroatom species, but also the binding motif is important for tuning the GNR electronic properties through doping.

Note that for the geometries of the above discussed GNRs the nitrogen atoms' lone pairs are orthogonal to the extended $\pi$-system of the GNR. By using heteroatoms such that their lone 
a.
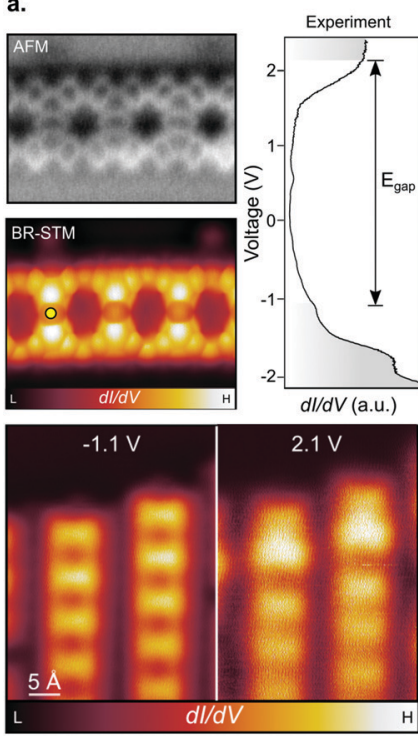

b.

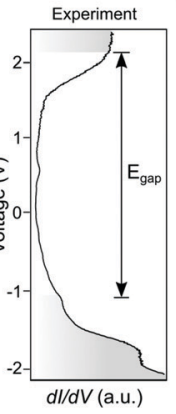$$
\text { 商 } 20
$$

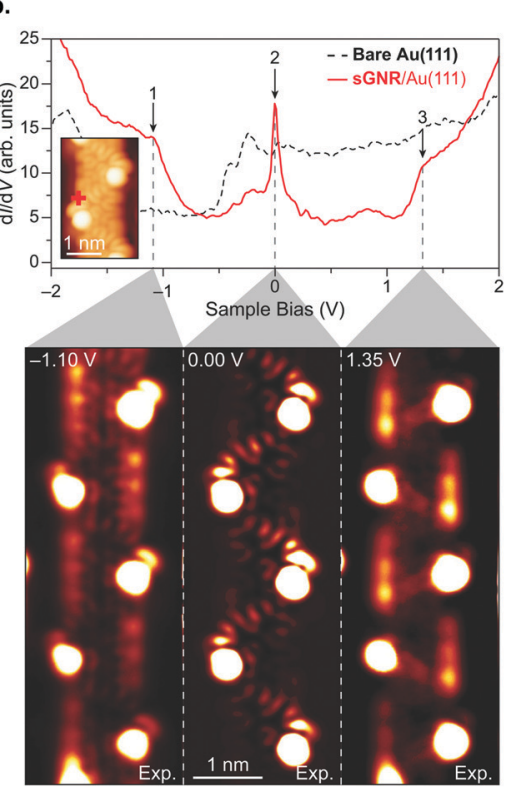

c.

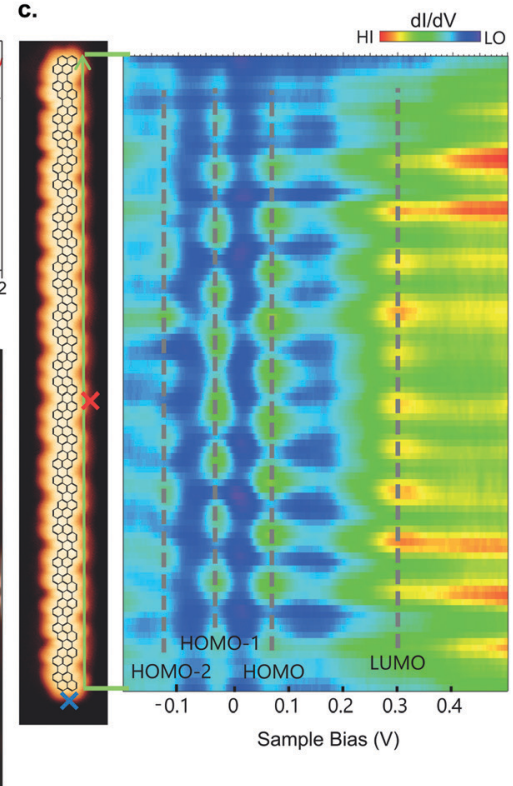

Fig. 8 GNRs with unconventional topologies. (a) Porous GNRs (70) hosting a regular array of vacancies. High-resolution nc-AFM und STM images (left), together with their electronic structure (right). Reprinted with permission from ref. 210. Copyright 2020, American Chemical Society. (b) Electronic structure of sawtooth GNRs (76) revealing a zero-mode band at the Fermi level (marked with 2 at 0 V). The red spectrum (top image) was taken at the position indicated in the inset. The three lower images display the $\mathrm{d} / / \mathrm{d} V$ maps recorded at the energies indicated by arrows in the top image. From ref. 41. Reprinted with permission from the American Association for the Advancement of Science. Copyright 2020, the Authors. (c) Electronic properties of pyrene-based GNRs (78) (STM image to the left) revealing their narrow band gap (line spectra to the right, taken along the green arrow given in the STM image). Reproduced with permission from ref. 213. Copyright 2020, Wiley-VCH.

pair overlaps with the extended $\pi$-system, the band gap of GNRs can be tuned, as well. ${ }^{200,215}$ For instance, cGNRs with either NH or $\mathrm{O}$ or $\mathrm{S}$ functionalization (39) have their band gap altered by approximately $0.2 \mathrm{eV}$ compared to a pristine cGNR (31) (Fig. 9b). ${ }^{200} \mathrm{~A}$ recent study conducted by Li et al. ${ }^{216}$ showed that edge functionalization can even act to partially depopulate the valence band through hole doping (Fig. 9c).

In addition to edge doping, heteroatoms may be placed on the inner part of the ribbon. In this case, the heteroatoms must conform to a trigonal planar structure to fit with graphene's geometry. This has only been applied in two structures so far: 23 and 25. In 23 (Fig. 9d), the empty boron $\mathrm{p}_{z}$ orbitals hybridize with the extended $\pi$-system of a 7-AGNR, leading to the formation of in-gap dopant bands with a band gap $E_{\mathrm{g}}=1.2 \mathrm{eV}^{217-219}$ Thus, the electronic properties of B-doped 7-AGNRs are significantly altered with respect to their pristine counterpart.

So far we considered heavily doped GNRs, with dopant concentrations far exceeding those in conventional semiconductors. By mixing undoped and doped precursors, a more dilute doping regime can be achieved. ${ }^{218,220}$ Individual boron dimers were recently found to induce $\pi$-paramagnetism in 7-AGNRs due to the local disruption of its extended $\pi$-system. ${ }^{221}$ A summary of the electronic band gaps and effective masses of the discussed GNRs is given in Table 1.

Heterostructures. GNR heterostructures can be fabricated through a variety of ways, such as co-deposition of multiple precursors, ${ }^{90,95,222}$ divergent on-surface synthesis, i.e. reactions with multiple possible reaction products, or lateral fusion of (structurally different) GNRs. ${ }^{115,223}$
For instance, co-deposition of $\mathbf{3 0}$ and $\mathbf{3 6}$ leads to stochastically formed p-n GNR heterojunctions with a shift of the conduction band of approximately $0.5 \mathrm{~V}$ across the junction area. ${ }^{96}$ This effect can be rationalized by the downshift of $0.1 \mathrm{eV}$ per nitrogen atom per monomer as discussed previously since 36 contains four $\mathrm{N}$ atoms. ${ }^{39}$

Instead of doping, the width-dependent electronic properties of GNRs can be harnessed to form molecular heterojunctions. One of the earliest examples of GNR heterojunctions was formed from 7-AGNRs and its only partially cyclodehydrogenated parent molecule. Thereby, the so-called $7-5^{+}$GNR heterojunctions form between the fully and partially cyclodehydrogenated sections. ${ }^{93}$ This was later expanded upon by Ma et al. ${ }^{224}$ who utilized voltage pulses in an STM to directly write patterns into the $5^{+}$sections.

Moreover, heterojunctions may even be formed from a single precursor molecule. Starting with a carbonyl-functionalized monomer (38, $\mathrm{X}=\mathrm{CO})$, thermal annealing induced scission of carbonyl groups leading to a type II heterojunction (Fig. 10a) ${ }^{92}$ Single precursor based heterojunctions can also be achieved through divergent on-surface synthesis, i.e. a single precursor may undergo a multitude of on-surface reactions resulting in multiple reaction products. ${ }^{91,225}$

From a fabrication point of view, technological applications of these heterojunctions are not yet in view because their formation is based on stochastic processes. It is thus possible that multiple heterojunctions may form within one ribbon. By using two types of precursor molecules with different halogen substitutions (e.g. Br and I) together with a 'linker molecule', which has both halogen substitutions, ribbons with preferentially 
a.
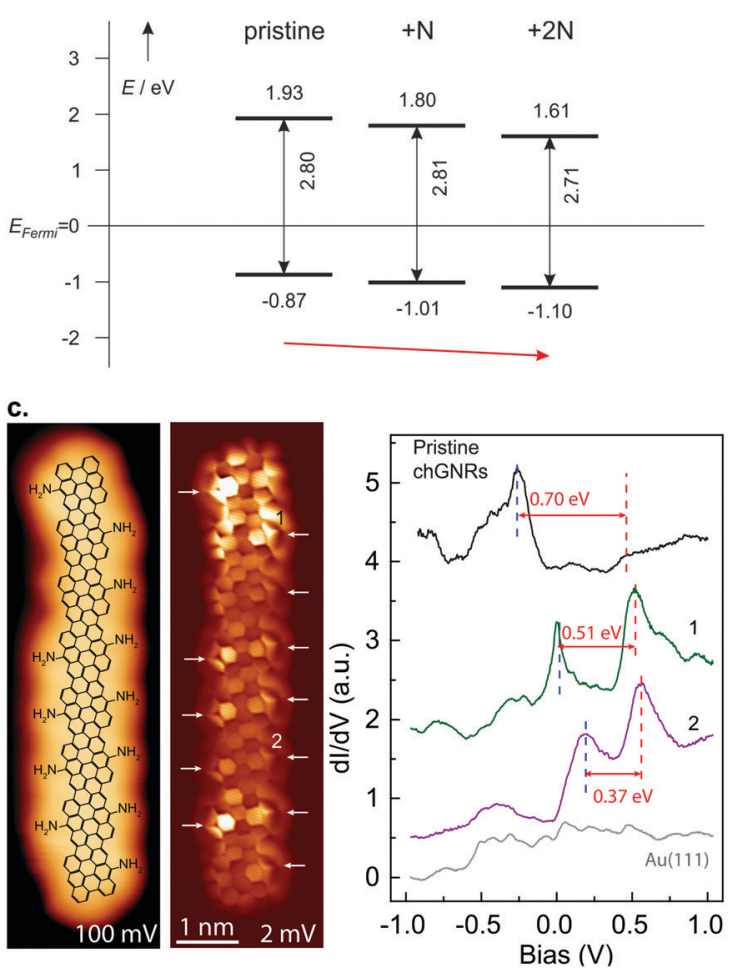

b.
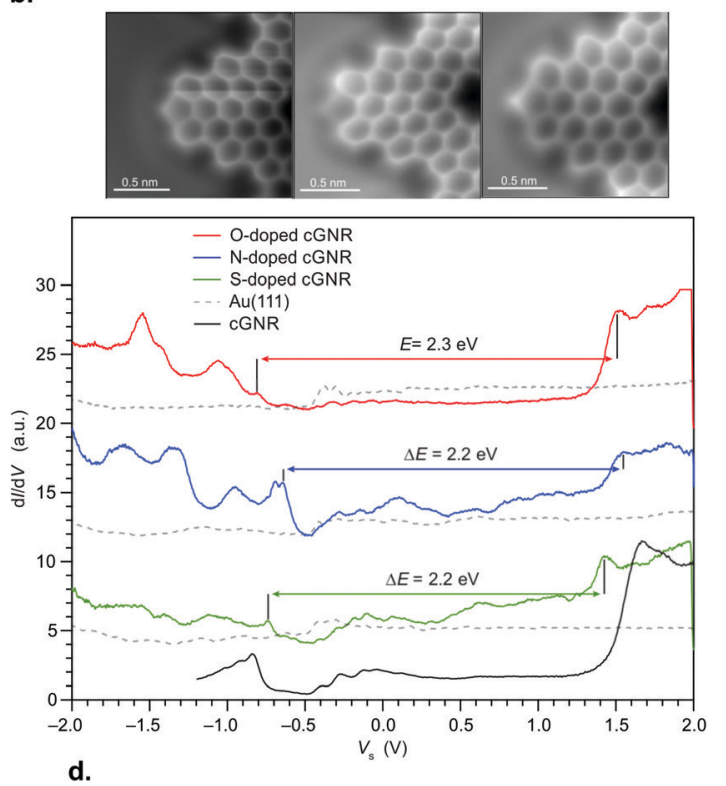

d.

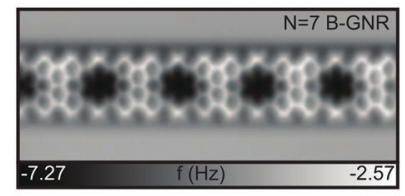

Fig. 9 (a) Summary of band alignment of nitrogen doped cGNRs on Au(111) investigated by a combination of HREELS and UPS. Pristine (2N) corresponds to 30 (34), while $1 \mathrm{~N}$ corresponds to a monomer with one pyridyl group. Reproduced with permission from ref. 39. Copyright 2013, Wiley-VCH. (b) Doping of cGNRs with (left to right) $\mathrm{NH}, \mathrm{O}$ and $\mathrm{S}$. The nc-AFM images detail the different appearances (left) while the right panel displays the respective STS spectra in comparison to a pristine cGNR. cGNR (doped cGNR) corresponds to 31 (39) in Fig. 3. Adapted with permission from ref. 200. Copyright 2017, American Chemical Society. (c) From left to right: STM image of an $\mathrm{NH}_{2}$ doped (3,1)-chGNR with overlaid structure model. Same area imaged with a CO-functionalized tip. STS spectra taken at the positions indicated in the STM image. Reprinted with permission from ref. 216. Copyright 2020, American Chemical Society. (d) nc-AFM image of a boron doped 7-AGNR. The boron substitutions appear dark in the image. Reproduced with permission from ref. 219. Copyright 2015, the Authors, originally published by Springer Nature. Provided under a Creative Commons CC BY 4.0 international license.

a single heterojunction may be formed as the two precursor molecules can only form a junction with the help of the linker molecule (Fig. 10b). ${ }^{89}$

Although research has in the beginning focused mainly on GNR-GNR junctions, a recent trend is co-deposition of small molecules to form heterostructures. ${ }^{226-229}$ Porphyrin derivatives are promising in this respect, as their central metal atom can be selected such that they possess a spin-polarized ground state. In addition, it has been shown that this spin state survives when contacted with GNRs (Fig. 10c). ${ }^{226}$ In addition, this spin state also survived in free-standing GNR-porphyrin heterostructures in two-probe conductance measurements. ${ }^{233}$ Recently, special nanographenes were found to host spin states, either due to sublattice imbalance ${ }^{212}$ or topological frustration. ${ }^{234-241}$ Thus, it may be possible to induce metal-free magnetism in nanographene-GNR heterostructures as well.

\section{Applications}

Almost a decade before the first successful synthesis of atomically precise GNRs, their electronic properties have been already predicted to be excellent for potential usage in nanoelectronic devices. ${ }^{28,29,146}$ While the previous sections deal with the synthesis and characterization of the electronic properties of GNRs, here we discuss the strategies to implement them in GNR-based devices, in particular field-effect transistors (FETs). Other applications are also under research (such as optoelectronics, ${ }^{228,242,243}$ gas sensors ${ }^{244}$ and DNA-sequencing ${ }^{245}$ ), but compared to FETs they are even more in their infancy.

\section{Ambient pressure chemical vapour deposition}

On the road to large-scale production of GNRs and GNR-based devices, the current need for an ultra-high vacuum (UHV) environment (pressure better than $10^{-7} \mathrm{mbar}$ ) for their synthesis is one of the roadblocks with respect to both expenses and throughput. ${ }^{246,247}$ The first step to fabrication in a more readily available environment was taken by Sakaguchi et al., ${ }^{246}$ who synthesized 5-, 7- and 9-AGNRs in a low-pressure ( $c a .1 \mathrm{mbar}$ ) argon atmosphere using a three-stage chemical vapor deposition (CVD) process, shown in Fig. 11a. The precursor material is evaporated from a quartz boat and passes into a two-zone furnace. In the first zone, kept at $350{ }^{\circ} \mathrm{C}$, the precursors collide with the quartz tube and dehalogenate. The biradicals then arrive at the $\mathrm{Au} /$ mica growth substrate in zone 2 , which is kept at $250{ }^{\circ} \mathrm{C}$ and polymerize. After 15 minutes, the temperature of zone 2 is increased to $400{ }^{\circ} \mathrm{C}$ to induce cyclodehydrogenation. The setup is 
Table 1 Overview of the experimentally obtained values for band gaps and effective masses for the various GNRs

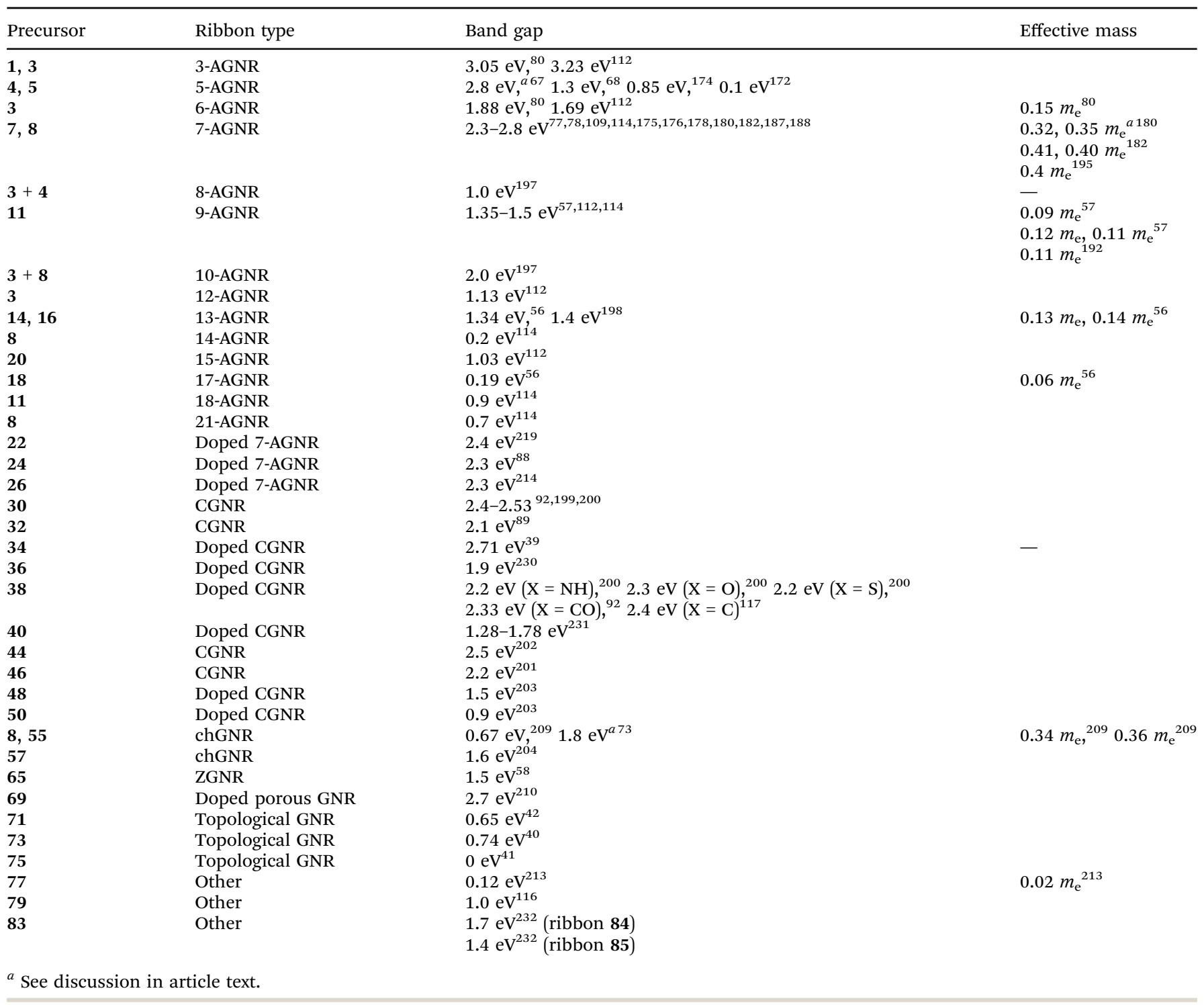

kept at a pressure of 1 torr of argon. These CVD-synthesized ribbons achieved lengths of up to $24 \mathrm{~nm}$ with an average length of around $10 \mathrm{~nm}$. It should be noted that the substrate is kept at a temperature sufficient to dehalogenate the monomers, whereas it is uncertain whether biradicals are actually formed by collision with the quartz tube. ${ }^{248}$ The process was developed further by Chen et $a .^{247}$ who were able to obtain 7- and 9-AGNRs as well as cGNRs (ribbon 31) with lengths up to $35 \mathrm{~nm}$ at ambient pressure, using a two-stage CVD process, omitting the high-temperature zone (see Fig. 11b) and increasing the pressure to atmospheric. The introduction of a small amount of hydrogen to the argon atmosphere during the synthesis was successfully shown to prevent the oxidation of the CVD-synthesized GNRs. ${ }^{247}$ XPS and HREELS data for the CVD-synthesized ribbons are comparable to UHV-synthesized GNRs, indicating that the composition of CVD-synthesized ribbons is the same as that of UHV-synthesized GNRs. The scalability of the approach is essentially only limited by the sample and setup dimensions. Optical images and Raman maps showed high uniformity over areas of square millimetres for transferred GNR films. However, the average length of the CVDsynthesized ribbons (around $10 \mathrm{~nm}$ ) is significantly shorter than the one obtained for UHV-synthesized ribbons of various types (between 20 and $45 \mathrm{~nm}^{77,249,250}$ ). A comparison of UHV- and CVD-synthesized GNR FETs shows better performance for the ones containing UHV-synthesized ribbons, likely due to the difference in length. ${ }^{251}$

Besides finding ways for upscaling the fabrication conditions, the long-term stability of the ribbons under ambient conditions is also of great interest and importance. Raman spectroscopy measurements demonstrated that GNRs were stable in air up to months, ${ }^{252}$ whereas GNR FETs only showed consistent behaviour over this period in UHV and their stability under ambient conditions was limited to a handful of days. ${ }^{247}$

\section{Aligned networks}

While it is possible to fabricate single-ribbon devices, from an experimental point of view this is challenging. ${ }^{38,253}$ With a 
a.
.
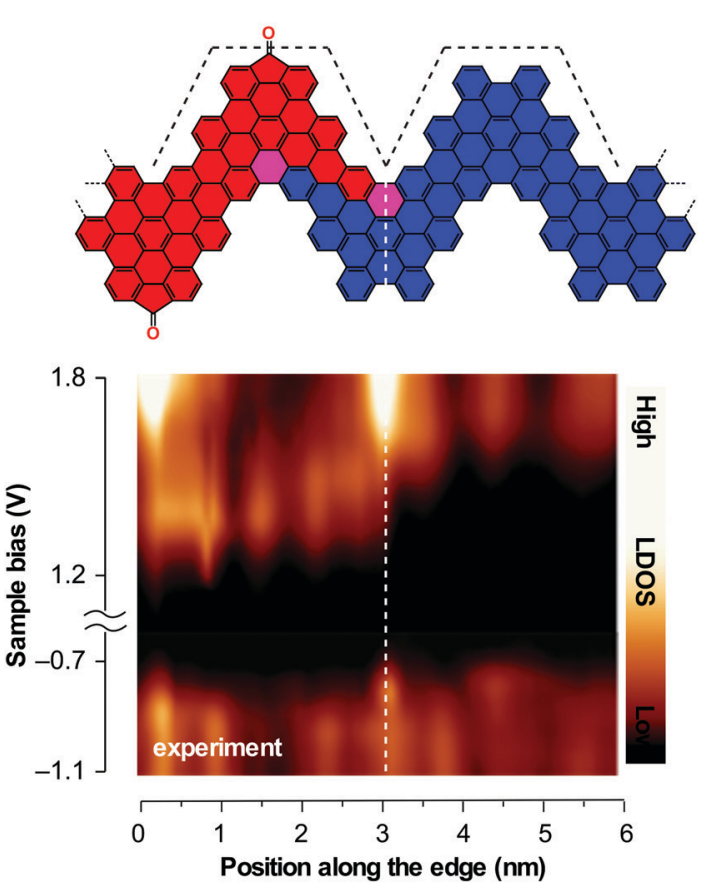

b.
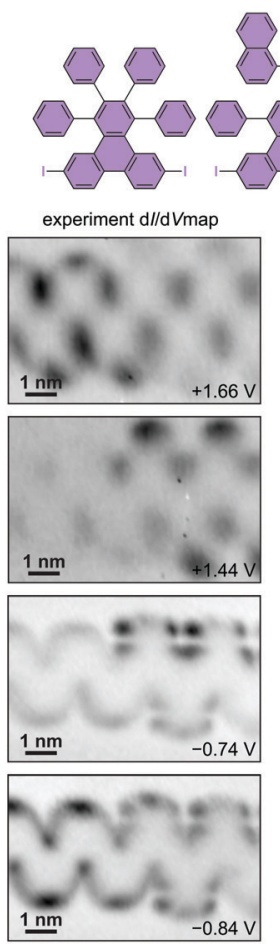

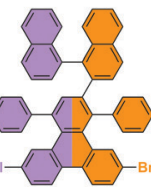

.
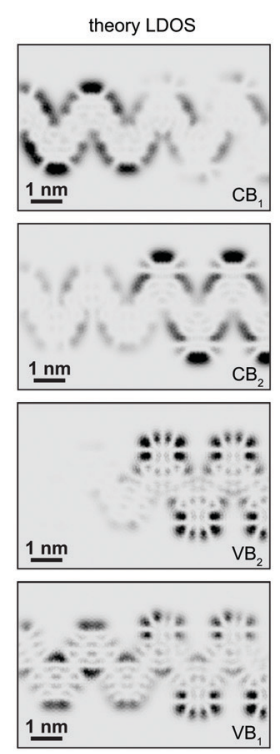

c.
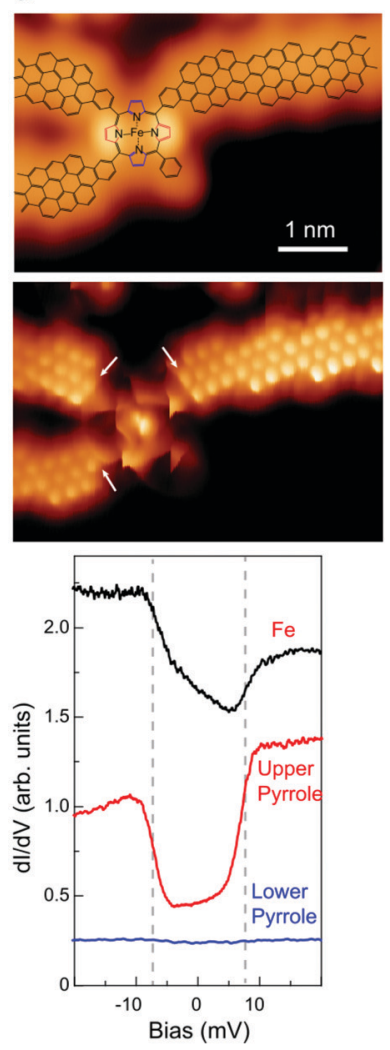

Fig. 10 GNR heterostructures. (a) Structural model of a heterojunction between carbonyl-functionalized and pristine chevron GNRs together with d//dV line spectra taken across the junction area. The formation of a type II heterojunction can be inferred from the $\mathrm{d} / / \mathrm{d} V$ spectra. Reproduced with permission from ref. 92. Copyright 2017, Springer Nature. (b) Hierarchical formation of GNR heterojunctions through the use of a linker molecule. Below the structural model, the $\mathrm{d} / / \mathrm{d} V$ maps on the junction area are shown together with the calculated LDOS. The $\mathrm{d} / / \mathrm{d} V$ maps reveal that the frontier states are confined to one side of the junction. Adapted with permission from ref. 89. Copyright 2018, American Chemical Society. (c) Junction between three (3,1) ch-GNRs and an Fe porphyrin derivative. $\mathrm{d} / / \mathrm{d} V$ spectra reveal that the spin state of the Fe atom is preserved upon contact with the GNRs. Reproduced with permission from ref. 226. Copyright 2018, the Authors, published by the American Association for the Advancement of Science. Provided under a Creative Commons CC BY-NC 4.0 international license.

fabrication success rate of around $10 \%$ it is simply infeasible for industrial applications. Moreover, a single ribbon will also not be able to meet the performance requirements for devices (e.g. sufficient drain current for field-effect transistors ${ }^{79,250}$ ). Therefore, a method is sought-after to produce networks of parallel aligned GNRs, as the resistance along the ribbon is up to three orders of magnitude lower than the resistance between ribbons ${ }^{254}$ (the same principle also constitutes the main drive behind recent work to prepare aligned arrays of carbon nanotubes ${ }^{255,256}$ ).

The synthesis of an aligned network of GNRs was first achieved by Linden $e$ al. ${ }^{77}$ by the use of a vicinal $\mathrm{Au}(788)$ surface. Linden et al. demonstrated on this substrate the synthesis of aligned 7-AGNRs and chevron GNRs (31) (Fig. 12). Later studies used polarized Raman spectroscopy to show that the alignment persists over areas of up to $150 \mu \mathrm{m}$ by $150 \mu \mathrm{m} .^{243}$

In addition to producing aligned, high-density arrays of GNRs, synthesis on a vicinal surface significantly reduces the occurrence of crosslinking defects. Lateral fusion, resulting in an array of GNRs with mixed width, is still possible but can mostly be avoided using sufficiently low temperatures during growth. ${ }^{79,251}$
Synthesis of unidirectionally aligned GNRs has also been demonstrated on the strongly anisotropic $\mathrm{Au}(110)$ surface, but the lower mobility on this surface results in shorter ribbons. ${ }^{76}$

\section{Transfer methods}

Regardless of the metal surface used to synthesize GNRs on, fabrication of any electronic device requires a transfer of the ribbons from the conducting metal surfaces to insulating ones, typically $\mathrm{SiO}_{2}$ on $\mathrm{Si}$. Transfer by bringing the synthesis and transfer substrate into direct mechanical contact results in a transfer of some of the ribbons ${ }^{37}$ but the reproducibility of this technique is low. ${ }^{38}$ Two other methods have been developed, in particular for transfer from thin $\mathrm{Au}(111)$ films deposited on mica or glass. The first of these methods involves immersing the GNR/Au/mica-or-glass stack in a hydrofluoric acid solution that delaminates the GNR/Au film from its substrate. The target substrate is then used to pick up the floating GNR/Au film, and the gold is etched by a KI/I $\mathrm{I}_{2}$ solution. ${ }^{38,246}$ The other common transfer method involves a poly-methyl methacrylate (PMMA) support layer, which is spin-coated onto the GNR/Au(111) sample. The mica and gold are removed as before, and then 
a.

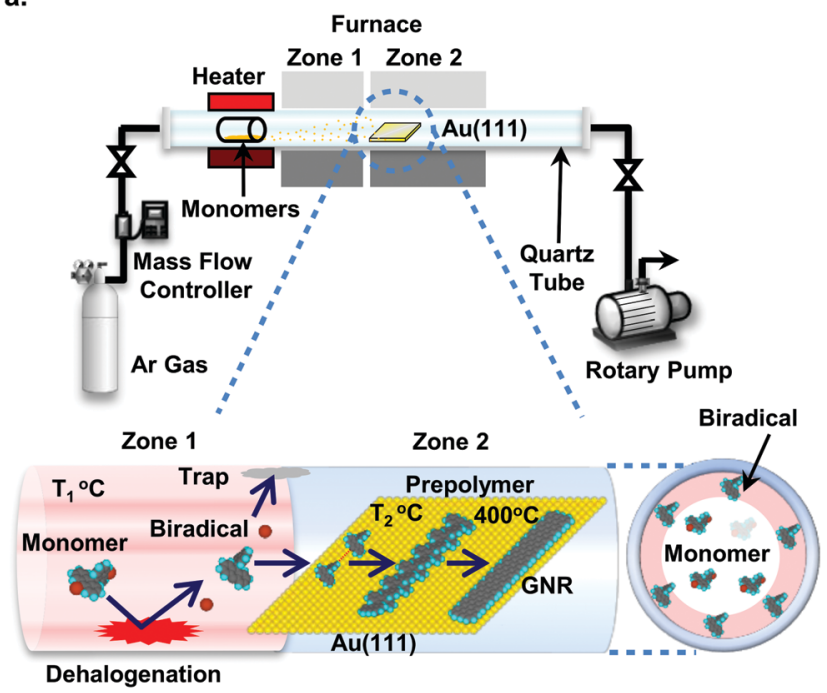

b.

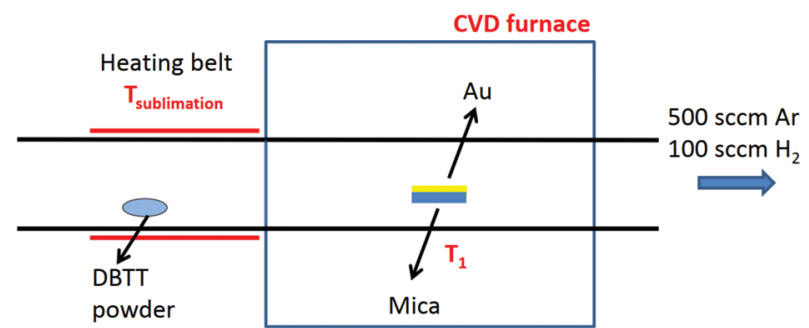

Fig. 11 Chemical vapour deposition setups. (a) Three-stage setup developed by Sakaguchi et al. It is evacuated by a rotary pump and fed with Argon gas to a pressure of 1 torr. Stage 1: the precursor molecules are placed in a quartz boat from which they are evaporated at $250^{\circ} \mathrm{C}$. Stage 2: the precursors collide with the quartz tube wall kept at $350{ }^{\circ} \mathrm{C}$ and are dehalogenated. Stage 3: the dehalogenated precursors arrive at the $\mathrm{Au}(111) /$ mica substrate, which is held at $250{ }^{\circ} \mathrm{C}$, and form polymer intermediates. These are converted to GNRs by raising the temperature of zone 2 to $400{ }^{\circ} \mathrm{C}$. Reproduced with permission from ref. 246. Copyright 2014, Wiley-VCH. (b) Two-stage setup developed by Chen et al., which is kept at ambient pressure in a mixed argon/ hydrogen atmosphere. Stage 1: the precursor is evaporated from a heating belt. Stage 2: the precursor is deposited on the Au/mica substrate, which is kept at $250^{\circ} \mathrm{C}$ to form polymer intermediates. The polymers are converted to GNRs by increasing the temperature to $450{ }^{\circ} \mathrm{C}$. Reprinted with permission from ref. 247. Copyright 2016, American Chemical Society.

the PMMA/GNR film is brought into contact with a $\mathrm{SiO}_{2}$ substrate. This stack is heated to remove residual water and finally rinsed in acetone to remove the PMMA. ${ }^{38,247,253,257}$ The GNRs transferred this way are unfortunately distributed and oriented randomly, which hinders device fabrication as noted above.

The vicinal surfaces used to synthesize aligned GNR networks are too expensive to be used only once since the etching step would dissolve them. ${ }^{79}$ However, modified versions of the support layer transfer method have been developed that preserve the Au(788) single crystal. For the first method, illustrated in Fig. 13, a hydrogen silsesquioxane (HSQ) layer is spin-coated on the GNR/Au(788) sample. The GNRs are then delaminated from the $\mathrm{Au}(788)$ substrate by intercalation of octanethiol (which strongly binds to noble metal surfaces ${ }^{258}$ ) and removed a.

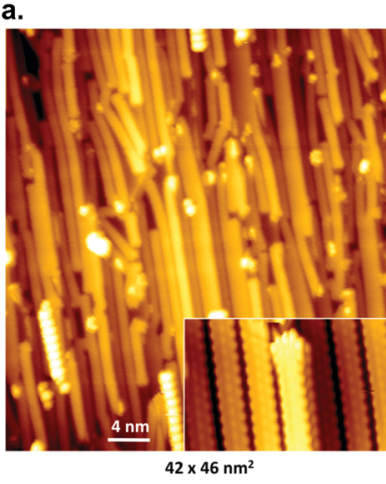

b.

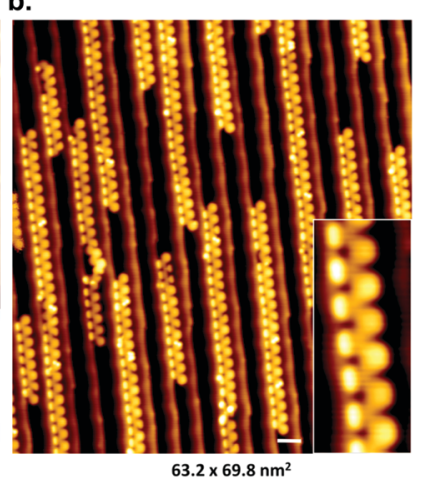

Fig. 12 STM images of aligned graphene nanoribbons on $A u(788)$, scale bars $4 \mathrm{~nm}$. (a) 7-AGNR and (b) cGNRs (31). Reprinted figure with permission from ref. 77. Copyright 2012 by the American Physical Society.

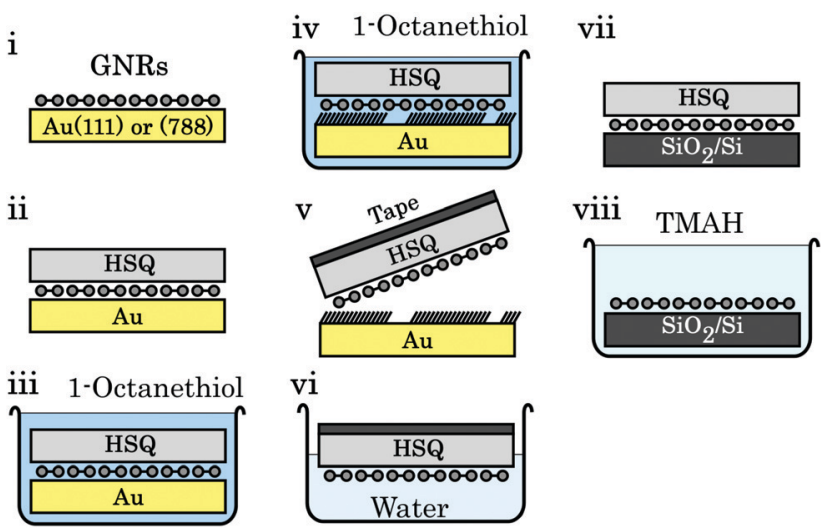

Fig. 13 Schematic of the octanethiol-intercalation transfer method. (i) Aligned GNRs on $A u$ (788). (ii) An HSQ support layer is spin-coated on the GNRs. (iii) The stack is submerged in an octanethiol solution. (iv) Octanethiol intercalates between GNRs and Au. (v) Thermal release tape is used to pick up the HSQ/GNR stack. (vi) Overnight immersion in water removes residual octanethiol molecules. (vii) The tape/HSQ/GNR stack is placed on $\mathrm{SiO}_{2} / \mathrm{Si}$; the tape is removed by annealing at $120{ }^{\circ} \mathrm{C}$. (viii) The HSQ support layer is removed by a $25 \%$ TMAH developer. Reprinted from ref. 79 , with the permission of AIP Publishing. Copyright 2018, the Authors.

from the gold surface using a thermal release tape. This stack is placed onto the $\mathrm{SiO}_{2} / \mathrm{Si}$ substrate, the tape is removed by annealing and the HSQ layer by using a tetramethylammonium (TMAH) developer. The second method ${ }^{243}$ is based on the bubble transfer method introduced for delaminating epitaxial graphene from $\mathrm{Cu}^{259}$ and $\mathrm{Pt}^{260}$ foil. A PMMA support layer is spin-coated on the GNR/Au(788) sample and then placed in an electrochemical cell containing $\mathrm{NaOH}$ solution. When the gold is biased negatively, hydrogen bubbles form at the gold-GNR interface which delaminate the PMMA/GNR stack. The latter can then be transferred to the target substrate and the PMMA is removed. Both methods result in an aligned GNR film on $\mathrm{SiO}_{2}$, though polarized Raman spectroscopy shows a decrease of alignment in the GNR array after the transfer step. ${ }^{79,243}$

Direct synthesis of the GNRs on insulating or semiconducting surfaces would eliminate the need for a transfer step, but challenges due to reasons highlighted above in the section on 
a.

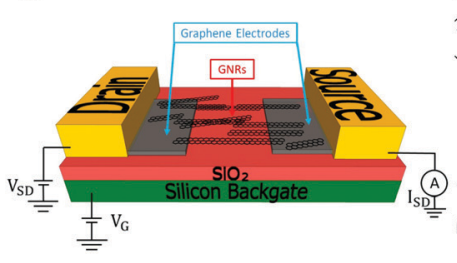

b.

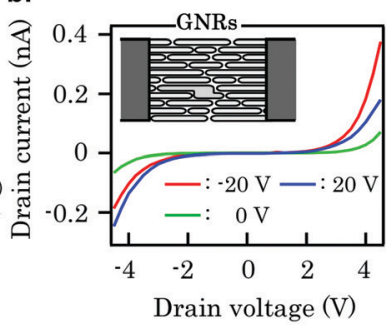

c.

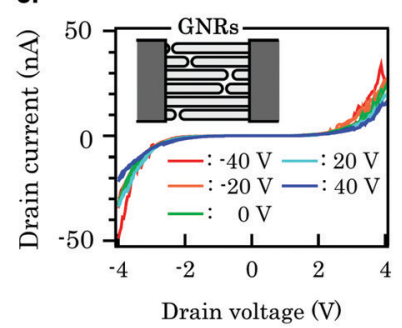

d.

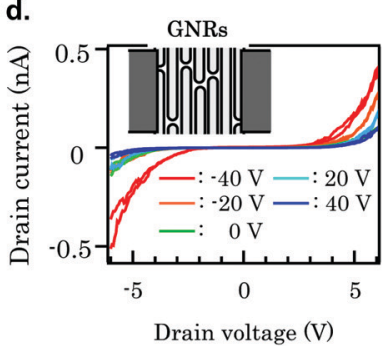

Fig. 14 (a) Schematic of a GNR-based field effect transistor with graphene electrodes. The drain and source are metal electrodes deposited on the graphene electrodes. Reprinted from ref. 251. Copyright 2019, with permission from Elsevier. (b-d) Comparison of drain current vs drain voltage characteristics for three 7-AGNR FETs. (b) FET with a channel length $L=60 \mathrm{~nm}$, width $W=50 \mu \mathrm{m}$, GNRs parallel to the channel. (c) FET with a channel length $L=20 \mathrm{~nm}, W=500 \mathrm{~nm}$, GNRs parallel to the channel. (d) FET with a channel length $L=20 \mathrm{~nm}, W=500 \mathrm{~nm}$, GNRs perpendicular to the channel. Reprinted from ref. 79, with the permission of AIP Publishing. Copyright 2018, the Authors.

Ullmann-type coupling, have prevented this possibility until very recently. ${ }^{109}$

\section{GNR field-effect transistor}

The first studies of GNR FETs focused on the behaviour of single GNRs in an FET contacted by metal source and drain electrodes. FETs are fabricated by transfer of randomly aligned GNRs to $\mathrm{SiO}_{2} / \mathrm{Si}$ substrate, after which metal electrodes are prepared by means of electron beam lithography and metal evaporation. Fabrication success rates for these devices were below $10 \%$ and no devices with a channel length over $30 \mathrm{~nm}$ (the average length of GNRs used in these studies) have so far been successfully fabricated. ${ }^{38}$ For functional devices, on/off current ratios from $10^{3}$ to $10^{5}$ are reported ${ }^{38,252,253}$ (approaching the typical on/off current ratios for conventional Si FETs, which range from $10^{6}$ to $10^{10}$ ). Often the reported on/off ratio did not scale as expected with the bandgap of the different GNRs employed, although this might be explained by different experimental conditions as GNR FET characteristics are highly variable from study to study. ${ }^{79}$ On the other hand, Martini et al. compared 5- and 9-AGNR FETs and found the trend in on/off current ratio to match with the bandgaps of the ribbons. ${ }^{251}$

Metal-contacted GNRs tend to be highly unstable p-type conductors in air, though might switch to n-type conduction after annealing in UHV. ${ }^{253}$ Longer channel lengths (up to $1 \mu \mathrm{m}$ ) are possible using a higher coverage of GNRs, ${ }^{247}$ but in any case device performance is limited by the Schottky-barrier contact between GNRs and metal electrodes.

Employment of graphene electrodes lowered the contact barrier, and resulted in nearly charge-neutral conducting devices regardless of environment. ${ }^{242,247,251}$ Two methods exist for preparing graphene electrodes. In the first, a graphene flake is transferred to $\mathrm{SiO}_{2}$ and a gap is opened by electroburning. ${ }^{251}$ In the second method, multilayer graphene is grown on SiC and subsequently etched into the desired shape. For both methods, the transfer of GNRs is the final step of the fabrication process. For a short channel length (up to $20 \mathrm{~nm}$ ) and a high density of GNRs on the target substrate, a much higher reproducibility could be achieved: $30 \%$ for CVD-synthesised ribbons and up to $45 \%$ for UHV-synthesised ribbons. ${ }^{247,251}$ This difference is attributed to the difference in average length for CVD- and
UHV-synthesised ribbons (there is no other difference in the structural quality of GNRs produced by each method ${ }^{38,247,257}$ ), as longer ribbons have a higher chance of bridging the gap between electrodes. FETs fabricated from UHV-synthesised GNRs also have a higher source-drain current. This, too, is because of the greater length of the GNRs, which leads to an increased contact area between the GNR and electrode and lowers the contact barrier.

A higher reproducibility is expected from FETs made with aligned GNR networks. Several 7-AGNR FETs ${ }^{79,250}$ have been fabricated, but the number of devices analysed is still too low to draw conclusions in a systematic way regarding the reproducibility. FETs with a channel shorter than average ribbon length show high source-drain currents while those with longer channels or ribbons aligned perpendicular to the channel show a lower source-drain current, see Fig. 14. The likely explanation for this is that the alignment of the ribbons is preserved during the device fabrication, and current is carried by GNRs spanning the channel for the first case, while in the latter two cases the resistance is dominated by ribbon-ribbon transport.

\section{Conclusions and outlook}

A decade of on-surface synthesis of GNRs has yielded a vast catalogue of synthesized and characterized GNRs, with various widths and edge structures. In this way, GNRs with varying electronic properties were synthesized, with a wide range of band gaps and in some cases even spin properties. ${ }^{174,261,262}$ The band gap of currently available GNRs ranges from approximately 0.12 to $2.4 \mathrm{eV},{ }^{182,213}$ the reported effective carrier masses range from 0.02 to $0.4 m_{\mathrm{e}^{195,213}}$ and it is very likely that newly synthesized GNRs will contribute to the extension of these ranges. The band alignment of GNRs can be effectively changed by atomically precise doping, which has only a minor impact on the band gap of the ribbons. This fine tuning of band alignment has been used to create various GNR heterojunctions, mostly based on cGNRS, in a facile manner. Since the electronic structure of GNRs is closely related to their width and edge termination, GNR heterojunctions can also be made by joining GNRs with different physical structures - something which is not possible for conventional heterostructures. GNRs offer 
great advantages over their parent material graphene, as they offer the possibility of having both a band gap and magnetic properties based only on their shape which is impossible for graphene. Whereas ten years ago the main focus of research was the synthesis of GNRs with various band gaps, now the field appears to be moving towards fabricating GNRs with unconventional edge structures, doping them to deliberately (fine) tune their properties and GNRs with additional phases, such as magnetic ${ }^{262}$ or topological-derived states. ${ }^{40-42}$

Although much progress was made in the past ten years, some challenges remain. The predicted spin properties at the zigzag edges of ZGNRs and chGNRs could so far not be experimentally verified and, relative to the wide range of AGNRs, only a few have been synthesized. In particular, more effort should be devoted to establishing protocols for the synthesis of different ZGNRs, as only one ZGNR has been experimentally obtained thus far. Since the synthesis of these ZGNRs and chGNRs is not easily realized utilizing the most commonly employed on-surface synthesis strategy based on Ullmann-type coupling, it will be advantageous to consider other on-surface reactions, or combinations thereof. To this end, knowledge from the on-surface synthesis of $1 \mathrm{D}$ and $2 \mathrm{D}$ structures can be borrowed - a research field which has already seen a number of instructive review articles. ${ }^{46-48}$ This way GNRs which are difficult to synthesize with Ullmann-type coupling may come into reach. In addition, some of these reactions may even be suited for fabricating GNRs on semiconducting or insulating substrates or facilitate the low-temperature synthesis of GNRs. In view of getting GNRs ready for applications, these are important prerequisites.

A particularly promising route to add more functionalities to GNRs is in the form of GNR-molecule heterostructures where the molecules add properties which the GNRs do not exhibit. A few examples of such heterostructures already exist. For GNRporphyrin junctions the spin state of the magnetic atom in the porphyrin core survived. ${ }^{226}$ In this way, magnetism can be added to GNRs. For GNR-nanographene junctions photoluminescence properties were reported. ${ }^{228}$ However, also other application possibilities are within reach. For instance, by using molecules which can undergo conformational changes mechanically moving contacts on the nanoscale are conceivable.

For moving towards device applications, it is imperative to improve ribbon length. With some notable exceptions, ${ }^{82,263}$ the typical length of on-surface synthesized GNRs is $20-45 \mathrm{~nm}$, much shorter than those fabricated with other techniques, such as solution synthesis ${ }^{10}$ or lithography, ${ }^{8}$ which can produce ribbons several microns long. Since intraribbon transport is up to three orders of magnitude more efficient ${ }^{254}$ than interribbon transport this limits device performance. To increase the ribbon length, ways have to be found to stop (i) premature bonding of hydrogen to the ribbon termini and (ii) unwanted cross-linking of ribbons, for instance via ribbon alignment.

The growth of aligned bottom-up GNRs on vicinal surfaces is expected to further enhance device performance and reliability, and has prompted the development of transfer methods that do not require dissolution of the (gold) substrate used for synthesis.
However, further research is needed to see whether aligned GNRs reliably perform better than unaligned ribbons grown on $\mathrm{Au} / \mathrm{mica}$ samples. Nonetheless, even if performance turns out to be improved by use of aligned networks synthesized on vicinal surfaces, their increased cost might still prevent adaptation in an industrial application compared to relatively cheap Au/mica substrates.

Device fabrication requires the GNRs to be on a semiconducting or insulating surface, so for any GNR grown on a metallic substrate a transfer step is a crucial part of the device fabrication process. Further research on the transfer methods might also result in improvements of device reliability, regardless of the specific substrate used for synthesis. Inspiration might be taken from e.g. iodine intercalation to decouple covalent networks from the substrate they were synthesized on ${ }^{264}$ or the myriad methods developed to transfer transition metal dichalcogenides ${ }^{265-268}$ or graphene. ${ }^{269,270}$ Direct growth on a desired semiconductor surface, as only recently demonstrated for $\mathrm{TiO}_{2}$, is also very promising, as it completely eliminates the need for a transfer.

Synthesis outside UHV should be explored further, as UHVsynthesis is a time-consuming and expensive process. Atomically precise GNRs are expected to become more technologically appealing when their synthesis may be carried out at higher pressures. First steps have been made in this regard with ambient-pressure CVD GNR synthesis, although the length of these GNRs is even shorter than those synthesized in UHV. ${ }^{247}$ Nevertheless, CVD synthesis seems to be the way forward for GNR-based devices, as the throughput of CVD synthesis will be drastically increased compared to UHV synthesis.

In conclusion, bottom-up synthesized GNRs are a promising candidate for device applications. If the above challenges are overcome, applications of GNRs will become increasingly practical and attractive. The rapid development of bottom-up GNRs also makes the prospect of future spintronic devices increasingly likely as well.

\section{Conflicts of interest}

The authors declare no competing interests.

\section{Acknowledgements}

This work was supported by the Netherlands Organization for Scientific Research (NWO) (Vici grant 680-47-633) and the Zernike Institute for Advanced Materials of the University of Groningen.

\section{Notes and references}

1 A. K. Geim and K. S. Novoselov, Nat. Mater., 2007, 6, 183-191. 2 D. G. Papageorgiou, I. A. Kinloch and R. J. Young, Prog. Mater. Sci., 2017, 90, 75-127.

3 A. H. Castro Neto, F. Guinea, N. M. R. Peres, K. S. Novoselov and A. K. Geim, Rev. Mod. Phys., 2009, 81, 109.

4 T. Zhang, S. Wu, R. Yang and G. Zhang, Front. Phys., 2017, 12, 127206. 
5 F. Schwierz, Nat. Nanotechnol., 2010, 5, 487.

6 K. Nakada, M. Fujita, G. Dresselhaus and M. S. Dresselhaus, Phys. Rev. B: Condens. Matter Mater. Phys., 1996, 54, 17954.

7 Z. Chen, Y. M. Lin, M. J. Rooks and P. Avouris, Phys. E, 2007, 40, 228-232.

8 M. Y. Han, B. Özyilmaz, Y. Zhang and P. Kim, Phys. Rev. Lett., 2007, 98, 206805.

9 X. Yang, X. Dou, A. Rouhanipour, L. Zhi, H. J. Räder and K. Müllen, J. Am. Chem. Soc., 2008, 130, 4216-4217.

10 X. Li, X. Wang, L. Zhang, S. Lee and H. Dai, Science, 2008, 319, 1229-1232.

11 A. Radocea, T. Sun, T. H. Vo, A. Sinitskii, N. R. Aluru and J. W. Lyding, Nano Lett., 2017, 17, 170-178.

12 T. H. Vo, M. Shekhirev, D. A. Kunkel, M. D. Morton, E. Berglund, L. Kong, P. M. Wilson, P. A. Dowben, A. Enders and A. Sinitskii, Nat. Commun., 2014, 5, 3189.

13 A. Narita, X. Y. Wang, X. Feng and K. Müllen, Chem. Soc. Rev., 2015, 44, 6616-6643.

14 A. D. Senese and W. A. Chalifoux, Molecules, 2019, 24, 118.

15 Y. Yano, N. Mitoma, H. Ito and K. Itami, J. Org. Chem., 2020, 85, 4-33.

16 K. Y. Yoon and G. Dong, Mater. Chem. Front., 2020, 4, 29-45.

17 D. V. Kosynkin, A. L. Higginbotham, A. Sinitskii, J. R. Lomeda, A. Dimiev, B. K. Price and J. M. Tour, Nature, 2009, 458, 872-876.

18 D. V. Kosynkin, W. Lu, A. Sinitskii, G. Pera, Z. Sun and J. M. Tour, ACS Nano, 2011, 5, 968-974.

19 C. Tao, L. Jiao, O. V. Yazyev, Y.-C. Chen, X. Zhang, R. B. Capaz, J. M. Tour, A. Zettl, H. Dai and M. F. Crommie, Nat. Phys., 2011, 7, 616-620.

20 L. Jiao, X. Wang, G. Diankov, H. Wang and H. Dai, Nat. Nanotechnol., 2010, 5, 321-325.

21 M. Sprinkle, M. Ruan, Y. Hu, J. Hankinson, M. Rubio-Roy, B. Zhang, X. Wu, C. Berger and W. A. De Heer, Nat. Nanotechnol., 2010, 5, 727-731.

22 J. Hicks, A. Tejeda, A. Taleb-Ibrahimi, M. S. Nevius, F. Wang, K. Shepperd, J. Palmer, F. Bertran, P. Le Fèvre, J. Kunc, W. A. De Heer, C. Berger and E. H. Conrad, Nat. Phys., 2013, 9, 49-54.

23 M. S. Nevius, F. Wang, C. Mathieu, N. Barrett, A. Sala, T. O. Menteş, A. Locatelli and E. H. Conrad, Nano Lett., 2014, 14, 6080-6086.

24 J. Baringhaus, J. Aprojanz, J. Wiegand, D. Laube, M. Halbauer, J. Hübner, M. Oestreich and C. Tegenkamp, Appl. Phys. Lett., 2015, 106, 043109.

25 A. Stöhr, J. Baringhaus, J. Aprojanz, S. Link, C. Tegenkamp, Y. Niu, A. A. Zakharov, C. Chen, J. Avila, M. C. Asensio and U. Starke, Ann. Phys., 2017, 529, 1700052.

26 J. Baringhaus, M. Ruan, F. Edler, A. Tejeda, M. Sicot, A. TalebIbrahimi, A. P. Li, Z. Jiang, E. H. Conrad, C. Berger, C. Tegenkamp and W. A. De Heer, Nature, 2014, 506, 349-354.

27 J. Aprojanz, S. R. Power, P. Bampoulis, S. Roche, A. P. Jauho, H. J. W. Zandvliet, A. A. Zakharov and C. Tegenkamp, Nat. Commun., 2018, 9, 4426.

28 V. Barone, O. Hod and G. E. Scuseria, Nano Lett., 2006, 6, 2748-2754.
29 Y. W. Son, M. L. Cohen and S. G. Louie, Phys. Rev. Lett., 2006, 97, 216803.

30 Y. Yoon and J. Guo, Appl. Phys. Lett., 2007, 91, 073103.

31 D. Querlioz, Y. Apertet, A. Valentin, K. Huet, A. Bournel, S. Galdin-Retailleau and P. Dollfus, Appl. Phys. Lett., 2008, 92, 042108.

32 D. Gunlycke, D. A. Areshkin and C. T. White, Appl. Phys. Lett., 2007, 90, 142104.

33 L. Talirz, P. Ruffieux and R. Fasel, Adv. Mater., 2016, 28, 6222-6231.

34 X. Zhou and G. Yu, Adv. Mater., 2020, 32, 1905957.

35 M. Corso, E. Carbonell-Sanromà and D. G. De Oteyza, in On-Surface Synthesis II, ed. D. G. De Oteyza and C. Rogero, Springer, Cham, 2016, pp. 113-152.

36 Z. Chen, A. Narita and K. Müllen, Adv. Mater., 2020, 32, 2001893.

37 J. Cai, P. Ruffieux, R. Jaafar, M. Bieri, T. Braun, S. Blankenburg, M. Muoth, A. P. Seitsonen, M. Saleh, X. Feng, K. Müllen and R. Fasel, Nature, 2010, 466, 470-473.

38 J. P. Llinas, A. Fairbrother, G. Borin Barin, W. Shi, K. Lee, S. Wu, B. Yong Choi, R. Braganza, J. Lear, N. Kau, W. Choi, C. Chen, Z. Pedramrazi, T. Dumslaff, A. Narita, X. Feng, K. Müllen, F. Fischer, A. Zettl, P. Ruffieux, E. Yablonovitch, M. Crommie, R. Fasel and J. Bokor, Nat. Commun., 2017, 8, 633.

39 C. Bronner, S. Stremlau, M. Gille, F. Brauße, A. Haase, S. Hecht and P. Tegeder, Angew. Chem., Int. Ed., 2013, 52, 4422-4425.

40 D. J. Rizzo, G. Veber, T. Cao, C. Bronner, T. Chen, F. Zhao, H. Rodriguez, S. G. Louie, M. F. Crommie and F. R. Fischer, Nature, 2018, 560, 204-208.

41 D. J. Rizzo, G. Veber, J. Jiang, R. McCurdy, T. Cao, C. Bronner, T. Chen, S. G. Louie, F. R. Fischer and M. F. Crommie, Science, 2020, 369, 1597-1603.

42 O. Gröning, S. Wang, X. Yao, C. A. Pignedoli, G. Borin Barin, C. Daniels, A. Cupo, V. Meunier, X. Feng, A. Narita, K. Müllen, P. Ruffieux and R. Fasel, Nature, 2018, 560, 209-213.

43 E. Costa Girão, L. Liang, E. Cruz-Silva, A. G. S. Filho and V. Meunier, Phys. Rev. Lett., 2011, 107, 135501.

44 F. Ullmann and J. Bielecki, Chem. Ber., 1901, 34, 2174-2185. 45 L. Grill, M. Dyer, L. Lafferentz, M. Persson, M. V. Peters and S. Hecht, Nat. Nanotechnol., 2007, 2, 687.

46 P. A. Held, H. Fuchs and A. Studer, Chem. - Eur. J., 2017, 23, 5874-5892.

47 L. Grill and S. Hecht, Nat. Chem., 2020, 12, 115-130.

48 S. Clair and D. G. De Oteyza, Chem. Rev., 2019, 119, 4717-4776.

49 M. Lackinger, Chem. Commun., 2017, 53, 7872-7885.

50 A. Batra, D. Cvetko, G. Kladnik, O. Adak, C. Cardoso, A. Ferretti, D. Prezzi, E. Molinari, A. Morgante and L. Venkataraman, Chem. Sci., 2014, 5, 4419-4423.

51 J. Björk, J. Phys.: Condens. Matter, 2016, 28, 083002.

52 J. Björk, F. Hanke and S. Stafström, J. Am. Chem. Soc., 2013, 135, 5768-5775.

53 M. T. Nguyen, C. A. Pignedoli and D. Passerone, Phys. Chem. Chem. Phys., 2011, 13, 154-160. 
54 R. Dorel and A. M. Echavarren, Eur. J. Org. Chem., 2017, 14-24.

55 F. R. Fischer, in From Polyphenylenes to Nanographenes and Graphene Nanoribbons, ed. K. Müllen and X. Feng, Springer, Cham, 2017, pp. 33-65.

56 J. Yamaguchi, H. Hayashi, H. Jippo, A. Shiotari, M. Ohtomo, M. Sakakura, N. Hieda, N. Aratani, M. Ohfuchi, Y. Sugimoto, H. Yamada and S. Sato, Commun. Mater., 2020, 1, 36.

57 L. Talirz, H. Söde, T. Dumslaff, S. Wang, J. R. SanchezValencia, J. Liu, P. Shinde, C. A. Pignedoli, L. Liang, V. Meunier, N. C. Plumb, M. Shi, X. Feng, A. Narita, K. Müllen, R. Fasel and P. Ruffieux, ACS Nano, 2017, 11, 1380-1388.

58 P. Ruffieux, S. Wang, B. Yang, C. Sanchez-Sanchez, J. Liu, T. Dienel, L. Talirz, P. Shinde, C. A. Pignedoli, D. Passerone, T. Dumslaff, X. Feng, K. Müllen and R. Fasel, Nature, 2016, 531, 489-492.

59 T. A. Pham, B. V. Tran, M. T. Nguyen and M. Stöhr, Small, 2017, 13, 1603675.

60 M. Bieri, M.-T. Nguyen, O. Gröning, J. Cai, M. Treier, K. AïtMansour, P. Ruffieux, C. A. Pignedoli, D. Passerone, M. Kastler, K. Müllen and R. Fasel, J. Am. Chem. Soc., 2010, 132, 16669-16676.

61 C. Morchutt, J. Björk, S. Krotzky, R. Gutzler and K. Kern, Chem. Commun., 2015, 51, 2440-2443.

62 T. Dienel, J. Gómez-Díaz, A. P. Seitsonen, R. Widmer, M. Iannuzzi, K. Radican, H. Sachdev, K. Müllen, J. Hutter and O. Gröning, ACS Nano, 2014, 8, 6571-6579.

63 W. Zhao, L. Dong, C. Huang, Z. M. Win and N. Lin, Chem. Commun., 2016, 52, 13225-13228.

64 M. Kittelmann, M. Nimmrich, R. Lindner, A. Gourdon and A. Kühnle, ACS Nano, 2013, 7, 5614-5620.

65 M. Kolmer, A. A. Ahmad Zebari, J. S. Prauzner-Bechcicki, W. Piskorz, F. Zasada, S. Godlewski, B. Such, Z. Sojka and M. Szymonski, Angew. Chem., Int. Ed., 2013, 52, 10300-10303.

66 P. Rahe, R. Lindner, M. Kittelmann, M. Nimmrich and A. Kühnle, Phys. Chem. Chem. Phys., 2012, 14, 6544-6548.

67 H. Zhang, H. Lin, K. Sun, L. Chen, Y. Zagranyarski, N. Aghdassi, S. Duhm, Q. Li, D. Zhong, Y. Li, K. Müllen, H. Fuchs and L. Chi, J. Am. Chem. Soc., 2015, 137, 4022-4025.

68 K. Sun, X. Li, L. Chen, H. Zhang and L. Chi, J. Phys. Chem. C, 2020, 124, 11422-11427.

69 P. Stoltze, J. Phys.: Condens. Matter, 1994, 6, 9495-9517.

70 P. Han, K. Akagi, F. Federici Canova, R. Shimizu, H. Oguchi, S. Shiraki, P. S. Weiss, N. Asao and T. Hitosugi, ACS Nano, 2015, 9, 12035-12044.

71 P. Han, K. Akagi, F. Federici Canova, H. Mutoh, S. Shiraki, K. Iwaya, P. S. Weiss, N. Asao and T. Hitosugi, ACS Nano, 2014, 8, 9181-9187.

72 C. Sánchez-Sánchez, T. Dienel, O. Deniz, P. Ruffieux, R. Berger, X. Feng, K. Müllen and R. Fasel, ACS Nano, 2016, 10, 8006-8011.

73 P. H. Jacobse, M. J. J. Mangnus, S. J. M. Zevenhuizen and I. Swart, ACS Nano, 2018, 12, 7048-7056.

74 S. Peljhan and A. Kokalj, Phys. Chem. Chem. Phys., 2011, 13, 20408-20417.
75 K. A. Simonov, N. A. Vinogradov, A. S. Vinogradov, A. V. Generalov, E. M. Zagrebina, G. I. Svirskiy, A. A. Cafolla, T. Carpy, J. P. Cunniffe, T. Taketsugu, A. Lyalin, N. Mårtensson and A. B. Preobrajenski, ACS Nano, 2015, 9, 8997-9011.

76 L. Massimi, O. Ourdjini, L. Lafferentz, M. Koch, L. Grill, E. Cavaliere, L. Gavioli, C. Cardoso, D. Prezzi, E. Molinari, A. Ferretti, C. Mariani and M. G. Betti, J. Phys. Chem. C, 2015, 119, 2427-2437.

77 S. Linden, D. Zhong, A. Timmer, N. Aghdassi, J. H. Franke, H. Zhang, X. Feng, K. Müllen, H. Fuchs, L. Chi and H. Zacharias, Phys. Rev. Lett., 2012, 108, 216801.

78 P. Ruffieux, J. Cai, N. C. Plumb, L. Patthey, D. Prezzi, A. Ferretti, E. Molinari, X. Feng, K. Müllen, C. A. Pignedoli and R. Fasel, ACS Nano, 2012, 6, 6930-6935.

79 M. Ohtomo, Y. Sekine, H. Hibino and H. Yamamoto, Appl. Phys. Lett., 2018, 112, 021602.

80 N. Merino-Díez, J. Lobo-Checa, P. Nita, A. Garcia-Lekue, A. Basagni, G. Vasseur, F. Tiso, F. Sedona, P. K. Das, J. Fujii, I. Vobornik, M. Sambi, J. I. Pascual, J. E. Ortega and D. G. De Oteyza, J. Phys. Chem. Lett., 2018, 9, 2510-2517.

81 G. Galeotti, M. Di Giovannantonio, J. Lipton-Duffin, M. Ebrahimi, S. Tebi, A. Verdini, L. Floreano, Y. FagotRevurat, D. F. Perepichka, F. Rosei and G. Contini, Faraday Discuss., 2017, 204, 453-469.

82 M. Di Giovannantonio, O. Deniz, J. I. Urgel, R. Widmer, T. Dienel, S. Stolz, C. Sánchez-Sánchez, M. Muntwiler, T. Dumslaff, R. Berger, A. Narita, X. Feng, K. Müllen, P. Ruffieux and R. Fasel, ACS Nano, 2018, 12, 74-81.

83 J. Eichhorn, D. Nieckarz, O. Ochs, D. Samanta, M. Schmittel, P. J. Szabelski and M. Lackinger, ACS Nano, 2014, 8, 7880-7889. 84 D. Peyrot and F. Silly, ACS Nano, 2016, 10, 5490-5498.

85 C. Bronner, T. Marangoni, D. J. Rizzo, R. A. Durr, J. H. Jørgensen, F. R. Fischer and M. F. Crommie, J. Phys. Chem. C, 2017, 121, 18490-18495.

86 P. H. Jacobse, A. van den Hoogenband, M. E. Moret, R. J. M. Klein Gebbink and I. Swart, Angew. Chem., Int. Ed., 2016, 55, 13052-13055.

87 L. Lafferentz, V. Eberhardt, C. Dri, C. Africh, G. Comelli, F. Esch, S. Hecht and L. Grill, Nat. Chem., 2012, 4, 215-220.

88 S. Kawai, S. Nakatsuka, T. Hatakeyama, R. Pawlak, T. Meier, J. Tracey, E. Meyer and A. S. Foster, Sci. Adv., 2018, 4, eaar7181.

89 C. Bronner, R. A. Durr, D. J. Rizzo, Y.-L. Lee, T. Marangoni, A. M. Kalayjian, H. Rodriguez, W. Zhao, S. G. Louie, F. R. Fischer and M. F. Crommie, ACS Nano, 2018, 12, 2193-2200.

90 P. H. Jacobse, A. Kimouche, T. Gebraad, M. M. Ervasti, J. M. Thijssen, P. Liljeroth and I. Swart, Nat. Commun., 2017, 8, 119.

91 D. J. Rizzo, M. Wu, H.-Z. Tsai, T. Marangoni, R. A. Durr, A. A. Omrani, F. Liou, C. Bronner, T. Joshi, G. D. Nguyen, G. F. Rodgers, W.-W. Choi, J. H. Jørgensen, F. R. Fischer, S. G. Louie and M. F. Crommie, Nano Lett., 2019, 19, 3221-3228.

92 G. D. Nguyen, H. Z. Tsai, A. A. Omrani, T. Marangoni, M. Wu, D. J. Rizzo, G. F. Rodgers, R. R. Cloke, R. A. Durr, 
Y. Sakai, F. Liou, A. S. Aikawa, J. R. Chelikowsky, S. G. Louie, F. R. Fischer and M. F. Crommie, Nat. Nanotechnol., 2017, 12, 1077.

93 S. Blankenburg, J. Cai, P. Ruffieux, R. Jaafar, D. Passerone, X. Feng, K. Müllen, R. Fasel and C. A. Pignedoli, ACS Nano, 2012, 6, 2020-2025.

94 K. Sun, O. Krejči, A. S. Foster, Y. Okuda, A. Orita and S. Kawai, J. Phys. Chem. C, 2019, 123, 17632-17638.

95 Y.-C. Chen, T. Cao, C. Chen, Z. Pedramrazi, D. Haberer, D. G. de Oteyza, F. R. Fischer, S. G. Louie and M. F. Crommie, Nat. Nanotechnol., 2015, 10, 156-160.

96 J. Cai, C. A. Pignedoli, L. Talirz, P. Ruffieux, H. Söde, L. Liang, V. Meunier, R. Berger, R. Li, X. Feng, K. Müllen and R. Fasel, Nat. Nanotechnol., 2014, 9, 896-900.

97 C. Bronner, J. Björk and P. Tegeder, J. Phys. Chem. C, 2015, 119, 486-493.

98 A. Mairena, M. Baljozovic, M. Kawecki, K. Grenader, M. Wienke, K. Martin, L. Bernard, N. Avarvari, A. Terfort, K. H. Ernst and C. Wäckerlin, Chem. Sci., 2019, 10, 2998-3004.

99 M. Abyazisani, J. M. MacLeod and J. Lipton-Duffin, ACS Nano, 2019, 13, 9270-9278.

100 B. V. Tran, T. A. Pham, M. Grunst, M. Kivala and M. Stöhr, Nanoscale, 2017, 9, 18305-18310.

101 R. Zuzak, A. Jančařík, A. Gourdon, M. Szymonski and S. Godlewski, ACS Nano, 2020, 14, 13316-13323.

102 K. Sun, T. Nishiuchi, K. Sahara, T. Kubo, A. S. Foster and S. Kawai, J. Phys. Chem. C, 2020, 124, 19675-19680.

103 L. Talirz, H. Söde, J. Cai, P. Ruffieux, S. Blankenburg, R. Jafaar, R. Berger, X. Feng, K. Müllen, D. Passerone, R. Fasel and C. A. Pignedoli, J. Am. Chem. Soc., 2013, 135, 2060-2063.

104 M. Treier, C. A. Pignedoli, T. Laino, R. Rieger, K. Müllen, D. Passerone and R. Fasel, Nat. Chem., 2011, 3, 61-67.

105 J. Björk, S. Stafström and F. Hanke, J. Am. Chem. Soc., 2011, 133, 14884-14887.

106 M. Koch, Z. Li, C. Nacci, T. Kumagai, I. Franco and L. Grill, Phys. Rev. Lett., 2018, 121, 47701.

107 P. Rempala, J. Kroulík and B. T. King, J. Org. Chem., 2006, 71, 5067-5081.

108 P. Rempala, J. Kroulík and B. T. King, J. Am. Chem. Soc., 2004, 126, 15002-15003.

109 M. Kolmer, A. K. Steiner, I. Izydorczyk, W. Ko, M. Engelund, M. Szymonski, A. P. Li and K. Amsharov, Science, 2020, 369, 571-575.

110 M. Kolmer, R. Zuzak, A. K. Steiner, L. Zajac, M. Engelund, S. Godlewski, M. Szymonski and K. Amsharov, Science, 2019, 363, 57-60.

111 A. Basagni, F. Sedona, C. A. Pignedoli, M. Cattelan, L. Nicolas, M. Casarin and M. Sambi, J. Am. Chem. Soc., 2015, 137, 1802-1808.

112 N. Merino-Díez, A. Garcia-Lekue, E. Carbonell-Sanromà, J. Li, M. Corso, L. Colazzo, F. Sedona, D. Sánchez-Portal, J. I. Pascual and D. G. De Oteyza, ACS Nano, 2017, 11, 11661-11668, DOI: 10.1021/acsnano.7b06765.

113 H. Huang, D. Wei, J. Sun, S. L. Wong, Y. P. Feng, A. H. C. Neto and A. T. S. Wee, Sci. Rep., 2012, 2, 983.
114 O. Deniz, C. Sánchez-Sánchez, T. Dumslaff, X. Feng, A. Narita, K. Müllen, N. Kharche, V. Meunier, R. Fasel and P. Ruffieux, Nano Lett., 2017, 17, 2197-2203.

115 S. Wang, N. Kharche, E. Costa Girão, X. Feng, K. Müllen, V. Meunier, R. Fasel and P. Ruffieux, Nano Lett., 2017, 17, 4277-4283.

116 C. Moreno, M. Vilas-Varela, B. Kretz, A. Garcia-Lekue, M. V. Costache, M. Paradinas, M. Panighel, G. Ceballos, S. O. Valenzuela, D. Peña and A. Mugarza, Science, 2018, 360, 199-203.

117 P. H. Jacobse, R. D. McCurdy, J. Jiang, D. J. Rizzo, G. Veber, P. Butler, R. Zuzak, S. G. Louie, F. R. Fischer and M. F. Crommie, J. Am. Chem. Soc., 2020, 142, 13507-13514.

118 L. Yang, C.-H. Park, Y.-W. Son, M. L. Cohen and S. G. Louie, Phys. Rev. Lett., 2007, 99, 186801.

119 K. Wakabayashi, K. I. Sasaki, T. Nakanishi and T. Enoki, Sci. Technol. Adv. Mater., 2010, 11, 054504.

120 M. Fujita, K. Wakabayashi, K. Nakada and K. Kusakabe, J. Phys. Soc. Jpn., 1996, 65, 1920-1923.

121 W. Huang, J. S. Wang and G. Liang, Phys. Rev. B: Condens. Matter Mater. Phys., 2011, 84, 045410.

122 L. Liang and V. Meunier, Appl. Phys. Lett., 2013, 102, 143101.

123 A. Lherbier, L. Liang, J. C. Charlier and V. Meunier, Carbon, 2015, 95, 833-842.

124 R. Denk, A. Lodi-Rizzini, S. Wang, M. Hohage, P. Zeppenfeld, J. Cai, R. Fasel, P. Ruffieux, R. F. J. Berger, Z. Chen, A. Narita, X. Feng, K. Müllen, R. Biagi, V. De Renzi, D. Prezzi, A. Ruini and A. Ferretti, Nanoscale, 2017, 9, 18326-18333.

125 S. Wang and J. Wang, J. Phys. Chem. C, 2012, 116, 10193-10197.

126 L. Liang and V. Meunier, J. Phys. Chem. C, 2015, 119, 775-783.

127 L. Liang and V. Meunier, Phys. Rev. B: Condens. Matter Mater. Phys., 2012, 86, 195404.

128 J. P. Joost, A. P. Jauho and M. Bonitz, Nano Lett., 2019, 19, 9045-9050.

129 T. Cao, F. Zhao and S. G. Louie, Phys. Rev. Lett., 2017, 119, 076401.

130 D.-X. Liu, X.-F. Li, X.-H. Zhang, X. Cao, X.-J. Zhu and D. Shi, J. Phys. Chem. C, 2020, 124, 15448-15453.

131 Y. L. Lee, F. Zhao, T. Cao, J. Ihm and S. G. Louie, Nano Lett., 2018, 18, 7247-7253.

132 R. Saito, M. Fujita, G. Dresselhaus and M. S. Dresselhaus, Phys. Rev. B: Condens. Matter Mater. Phys., 1992, 46, 1804.

133 H. Lee, Y. W. Son, N. Park, S. Han and J. Yu, Phys. Rev. B: Condens. Matter Mater. Phys., 2005, 72, 174431.

134 J. Fernández-Rossier, Phys. Rev. B: Condens. Matter Mater. Phys., 2008, 77, 075430.

135 O. V. Yazyev, R. B. Capaz and S. G. Louie, Phys. Rev. B: Condens. Matter Mater. Phys., 2011, 84, 115406.

136 A. R. Carvalho, J. H. Warnes and C. H. Lewenkopf, Phys. Rev. B: Condens. Matter Mater. Phys., 2014, 89, 245444.

137 Z. Jiang and Y. Song, Phys. B, 2015, 464, 61-67.

138 M. Ezawa, Phys. Rev. B: Condens. Matter Mater. Phys., 2006, 73, 045432.

139 M. Ezawa, Phys. Status Solidi, 2007, 4, 489-492. 
140 E. Costa Girão, E. Cruz-Silva, L. Liang, A. G. S. Filho and V. Meunier, Phys. Rev. B: Condens. Matter Mater. Phys, 2012, 85, 235431.

141 L. Liang, E. C. Girão and V. Meunier, Phys. Rev. B: Condens. Matter Mater. Phys., 2013, 88, 035420.

142 D. Prezzi, D. Varsano, A. Ruini and E. Molinari, Phys. Rev. B: Condens. Matter Mater. Phys., 2011, 84, 041401.

143 D. Prezzi, D. Varsano, A. Ruini, A. Marini and E. Molinari, Phys. Rev. B: Condens. Matter Mater. Phys., 2008, 77, 041404.

144 N. Kharche and V. Meunier, J. Phys. Chem. Lett., 2016, 7, 1526-1533.

145 J. B. Neaton, M. S. Hybertsen and S. G. Louie, Phys. Rev. Lett., 2006, 97, 216405.

146 K. Wakabayashi, Phys. Rev. B: Condens. Matter Mater. Phys., 2001, 64, 125428.

147 E. C. Girão, E. Cruz-Silva and V. Meunier, ACS Nano, 2012, 6, 6483-6491.

148 L. Salemi, A. Lherbier and J. C. Charlier, Phys. Rev. B, 2018, 98, 214204.

149 A. Lherbier, B. Biel, Y. M. Niquet and S. Roche, Phys. Rev. Lett., 2008, 100, 036803.

150 F. Sánchez-Ochoa, J. Zhang, Y. Du, Z. Huang, G. Canto, M. Springborg and G. H. Cocoletzi, Phys. Chem. Chem. Phys., 2019, 21, 24867-24875.

151 L. L. Cui, M. Q. Long, X. J. Zhang, X. M. Li, D. Zhang and B. C. Yang, Phys. Lett. A, 2016, 380, 730-738.

152 D. A. Areshkin and B. K. Nikolić, Phys. Rev. B: Condens. Matter Mater. Phys., 2010, 81, 155450.

153 T. B. Martins, R. H. Miwa, A. J. R. Da Silva and A. Fazzio, Phys. Rev. Lett., 2007, 98, 196803.

154 M. Pizzochero, K. Černevičs, G. B. Barin, S. Wang, P. Ruffieux, R. Fasel and O. V. Yazyev, 2020, arXiv:2006.15075.

155 P. H. De Oliveira Neto, J. F. Teixeira, W. F. Da Cunha, R. Gargano and G. M. E. Silva, J. Phys. Chem. Lett., 2012, 3, 3039-3042.

156 M. F. C. Fobasso, C. Kenfack-Sadem, E. Baloitcha, A. J. Fotué and L. C. Fai, Eur. Phys. J. Plus, 2020, 135, 471.

157 L. A. Ribeiro, W. F. Da Cunha, A. L. De Almeida Fonseca, G. M. e Silva and S. Stafström, J. Phys. Chem. Lett., 2015, 6, 510-514.

158 W. Ferreira Da Cunha, P. H. De Oliveira Neto, A. Terai and G. M. e Silva, Phys. Rev. B, 2016, 94, 014301.

159 G. G. Silva, L. A. Ribeiro Junior, M. L. Pereira Junior, A. L. de Almeida Fonseca, R. T. de Sousa-Júnior and G. M. e Silva, Sci. Rep., 2019, 9, 11994.

160 M. L. Pereira Júnior, G. M. e Silva and L. A. Ribeiro Junior, J. Phys. Chem. Lett., 2020, 11, 5538-5543.

161 M. M. Fischer, L. A. Ribeiro Junior, W. F. da Cunha, L. E. de Sousa, G. M. e Silva and P. H. de Oliveira Neto, Carbon, 2020, 158, 553-558.

162 C. Kenfack-Sadem, M. F. C. Fobasso, F. Amo-Mensah, E. Baloitcha, A. Fotué and L. C. Fai, Physica E, 2020, 122, 114154.

163 M. L. Pereira Júnior, W. F. Da Cunha, R. T. De Sousa Junior, W. F. Giozza, G. M. e Silva and L. A. Ribeiro, J. Phys. Chem. C, 2020, 124, 22392-22398.
164 Y. Sakai and A. Terai, J. Phys. Soc. Jpn., 2019, 88, 054718.

165 M. L. Pereira Júnior, B. G. Enders Neto, W. F. Giozza, R. T. Sousa Júnior, G. M. e Silva and L. A. Ribeiro Júnior, J. Mater. Chem. C, 2020, 8, 12100-12107.

166 N. Richter, Z. Chen, A. Tries, T. Prechtl, A. Narita, K. Müllen, K. Asadi, M. Bonn and M. Kläui, Sci. Rep., 2020, 10, 1988.

167 N. J. Van Der Kaap, I. Katsouras, K. Asadi, P. W. M. Blom, L. J. A. Koster and D. M. De Leeuw, Phys. Rev. B, 2016, 93, 140206(R).

168 K. Asadi, A. J. Kronemeijer, T. Cramer, L. Jan Anton Koster, P. W. M. Blom and D. M. De Leeuw, Nat. Commun., 2013, 4, 1710.

169 G. Vasseur, Y. Fagot-Revurat, M. Sicot, B. Kierren, L. Moreau, D. Malterre, L. Cardenas, G. Galeotti, J. Lipton-Duffin, F. Rosei, M. Di Giovannantonio, G. Contini, P. Le Fèvre, F. Bertran, L. Liang, V. Meunier and D. F. Perepichka, Nat. Commun., 2016, 7, 10235.

170 J. A. Lipton-Duffin, O. Ivasenko, D. F. Perepichka and F. Rosei, Small, 2009, 5, 592-597.

171 N. Abdurakhmanova, N. Amsharov, S. Stepanow, M. Jansen, K. Kern and K. Amsharov, Carbon, 2014, 77, 1187-1190.

172 A. Kimouche, M. M. Ervasti, R. Drost, S. Halonen, A. Harju, P. M. Joensuu, J. Sainio and P. Liljeroth, Nat. Commun., 2015, 6, 10177.

173 A. D. Zdetsis and E. N. Economou, Carbon, 2017, 116, 422-434.

174 J. Lawrence, P. Brandimarte, A. Berdonces-Layunta, M. S. G. Mohammed, A. Grewal, C. C. Leon, D. Sánchez-Portal and D. G. De Oteyza, ACS Nano, 2020, 14, 4499-4508.

175 Y. Que, B. Liu, Y. Zhuang, C. Xu, K. Wang and X. Xiao, J. Phys. Chem. Lett., 2020, 11, 5044-5050.

176 L. Talirz, H. Söde, S. Kawai, P. Ruffieux, E. Meyer, X. Feng, K. Müllen, R. Fasel, C. A. Pignedoli and D. Passerone, ChemPhysChem, 2019, 20, 2348-2353.

177 M. C. Chong, N. Afshar-Imani, F. Scheurer, C. Cardoso, A. Ferretti, D. Prezzi and G. Schull, Nano Lett., 2018, 18, 175-181.

178 M. Koch, F. Ample, C. Joachim and L. Grill, Nat. Nanotechnol., 2012, 7, 713-717.

179 J. Van Der Lit, M. P. Boneschanscher, D. Vanmaekelbergh, M. Ijäs, A. Uppstu, M. Ervasti, A. Harju, P. Liljeroth and I. Swart, Nat. Commun., 2013, 4, 2023.

180 S. Wang, L. Talirz, C. A. Pignedoli, X. Feng, K. Müllen, R. Fasel and P. Ruffieux, Nat. Commun., 2016, 7, 11507.

181 C. Ma, Z. Xiao, H. Zhang, L. Liang, J. Huang, W. Lu, B. G. Sumpter, K. Hong, J. Bernholc and A. P. Li, Nat. Commun., 2017, 8, 14815.

182 H. Söde, L. Talirz, O. Gröning, C. A. Pignedoli, R. Berger, X. Feng, K. Müllen, R. Fasel and P. Ruffieux, Phys. Rev. B: Condens. Matter Mater. Phys., 2015, 91, 045429.

183 K. A. Simonov, A. V. Generalov, A. S. Vinogradov, G. I. Svirskiy, A. A. Cafolla, C. McGuinness, T. Taketsugu, A. Lyalin, N. Mårtensson and A. B. Preobrajenski, Sci. Rep., 2018, 8, 3506 .

184 P. H. Jacobse, K. A. Simonov, M. J. J. Mangnus, G. I. Svirskiy, A. V. Generalov, A. S. Vinogradov, A. Sandell, 
N. Mårtensson, A. B. Preobrajenski and I. Swart, J. Phys. Chem. C, 2019, 123, 8892-8901.

185 G. Borin Barin, A. Fairbrother, L. Rotach, M. Bayle, M. Paillet, L. Liang, V. Meunier, R. Hauert, T. Dumslaff, A. Narita, K. Müllen, H. Sahabudeen, R. Berger, X. Feng, R. Fasel and P. Ruffieux, ACS Appl. Nano Mater., 2019, 2, 2184-2192.

186 S. Zhao, G. B. Barin, T. Cao, J. Overbeck, R. Darawish, T. Lyu, S. Drapcho, S. Wang, T. Dumslaff, A. Narita, M. Calame, K. Müllen, S. G. Louie, P. Ruffieux, R. Fasel and F. Wang, Nano Lett., 2020, 20, 1124-1130.

187 C. Bronner, F. Leyssner, S. Stremlau, M. Utecht, P. Saalfrank, T. Klamroth and P. Tegeder, Phys. Rev. B: Condens. Matter Mater. Phys., 2012, 86, 085444.

188 C. Bronner, M. Utecht, A. Haase, P. Saalfrank, T. Klamroth and P. Tegeder, J. Chem. Phys., 2014, 140, 024701.

189 R. Denk, M. Hohage, P. Zeppenfeld, J. Cai, C. A. Pignedoli, H. Söde, R. Fasel, X. Feng, K. Müllen, S. Wang, D. Prezzi, A. Ferretti, A. Ruini, E. Molinari and P. Ruffieux, Nat. Commun., 2014, 5, 4253.

190 J. M. Garcia-Lastra and K. S. Thygesen, Phys. Rev. Lett., 2011, 106, 187402.

191 B. V. Senkovskiy, A. V. Fedorov, D. Haberer, M. Farjam, K. A. Simonov, A. B. Preobrajenski, N. Mårtensson, N. Atodiresei, V. Caciuc, S. Blügel, A. Rosch, N. I. Verbitskiy, M. Hell, D. V. Evtushinsky, R. German, T. Marangoni, P. H. M. van Loosdrecht, F. R. Fischer and A. Grüneis, Adv. Electron. Mater., 2017, 3, 1600490.

192 B. V. Senkovskiy, D. Haberer, D. Y. Usachov, A. V. Fedorov, N. Ehlen, M. Hell, L. Petaccia, G. Di Santo, R. A. Durr, F. R. Fischer and A. Grüneis, Phys. Status Solidi RRL, 2017, 11, 1700157.

193 A. Bergvall and T. Löfwander, Phys. Rev. B: Condens. Matter Mater. Phys., 2013, 87, 205431.

194 L. Bürgi, L. Petersen, H. Brune and K. Kern, Surf. Sci., 2000, 447, L157-L161.

195 B. V. Senkovskiy, D. Y. Usachov, A. V. Fedorov, D. Haberer, N. Ehlen, F. R. Fischer and A. Grüneis, 2D Mater., 2018, 5, 035007.

196 N. J. Tao, Nat. Nanotechnol., 2006, 1, 173-181.

197 K. Sun, P. Ji, J. Zhang, J. Wang, X. Li, X. Xu, H. Zhang and L. Chi, Small, 2019, 15, 1804526.

198 Y.-C. Chen, D. G. de Oteyza, Z. Pedramrazi, C. Chen, F. R. Fischer and M. F. Crommie, ACS Nano, 2013, 7, 6123-6128.

199 O. Deniz, C. Sánchez-Sánchez, R. Jaafar, N. Kharche, L. Liang, V. Meunier, X. Feng, K. Müllen, R. Fasel and P. Ruffieux, Chem. Commun., 2018, 54, 1619-1622.

200 R. A. Durr, D. Haberer, Y.-L. Lee, R. Blackwell, A. M. Kalayjian, T. Marangoni, J. Ihm, S. G. Louie and F. R. Fischer, J. Am. Chem. Soc., 2018, 140, 807-813.

201 J. D. Teeter, P. Zahl, M. Mehdi Pour, P. S. Costa, A. Enders and A. Sinitskii, ChemPhysChem, 2019, 20, 2281-2285.

202 M. Shekhirev, P. Zahl and A. Sinitskii, ACS Nano, 2018, 12, 8662-8669.

203 Y. Fu, H. Yang, Y. Gao, L. Huang, R. Berger, J. Liu, H. Lu, Z. Cheng, S. Du, H. J. Gao and X. Feng, Angew. Chem., Int. Ed., 2020, 59, 8873-8879.
204 A. Keerthi, C. Sánchez-Sánchez, O. Deniz, P. Ruffieux, D. Schollmeyer, X. Feng, A. Narita, R. Fasel and K. Müllen, Chem. - Asian J., 2020, 15, 3807-3811.

205 K. A. Simonov, N. A. Vinogradov, A. S. Vinogradov, A. V. Generalov, E. M. Zagrebina, N. Mårtensson, A. A. Cafolla, T. Carpy, J. P. Cunniffe and A. B. Preobrajenski, J. Phys. Chem. C, 2014, 118, 12532-12540.

206 P. Han, K. Akagi, F. Federici Canova, H. Mutoh, S. Shiraki, K. Iwaya, P. S. Weiss, N. Asao and T. Hitosugi, ACS Nano, 2015, 9, 3404-3405.

207 K. A. Simonov, N. A. Vinogradov, A. S. Vinogradov, A. V. Generalov, E. M. Zagrebina, N. Mårtensson, A. A. Cafolla, T. Carpy, J. P. Cunniffe and A. B. Preobrajenski, ACS Nano, 2015, 9, 3399-3403.

208 F. Schulz, P. H. Jacobse, F. F. Canova, J. van der Lit, D. Z. Gao, A. van den Hoogenband, P. Han, R. J. M. Klein Gebbink, M.-E. Moret, P. M. Joensuu, I. Swart and P. Liljeroth, J. Phys. Chem. C, 2017, 121, 2896-2904.

209 N. Merino-Díez, J. Li, A. Garcia-Lekue, G. Vasseur, M. VilasVarela, E. Carbonell-Sanromà, M. Corso, J. E. Ortega, D. Peña, J. I. Pascual and D. G. De Oteyza, J. Phys. Chem. Lett., 2018, 9, 25-30.

210 R. Pawlak, X. Liu, S. Ninova, P. D’Astolfo, C. Drechsel, S. Sangtarash, R. Häner, S. Decurtins, H. Sadeghi, C. J. Lambert, U. Aschauer, S. X. Liu and E. Meyer, J. Am. Chem. Soc., 2020, 142, 12568-12573.

211 J. J. Palacios, J. Fernández-Rossier and L. Brey, Phys. Rev. B: Condens. Matter Mater. Phys., 2008, 77, 195428.

212 E. H. Lieb, Phys. Rev. Lett., 1989, 62, 1201-1204.

213 Q. Sun, O. Gröning, J. Overbeck, O. Braun, M. L. Perrin, G. Borin Barin, M. El Abbassi, K. Eimre, E. Ditler, C. Daniels, V. Meunier, C. A. Pignedoli, M. Calame, R. Fasel, P. Ruffieux, G. B. Barin, M. El Abbassi, K. Eimre, E. Ditler, C. Daniels, V. Meunier, C. A. Pignedoli, M. Calame, R. Fasel and P. Ruffieux, Adv. Mater., 2020, 32, 1906054.

214 E. Carbonell-Sanromà, J. Hieulle, M. Vilas-Varela, P. Brandimarte, M. Iraola, A. Barragán, J. Li, M. Abadia, M. Corso, D. Sánchez-Portal, D. Peña and J. I. Pascual, ACS Nano, 2017, 11, 7355-7361.

215 G. D. Nguyen, F. M. Toma, T. Cao, Z. Pedramrazi, C. Chen, D. J. Rizzo, T. Joshi, C. Bronner, Y.-C. Chen, M. Favaro, S. G. Louie, F. R. Fischer and M. F. Crommie, J. Phys. Chem. C, 2016, 120, 2684-2687.

216 J. Li, P. Brandimarte, M. Vilas-Varela, N. Merino-Díez, C. Moreno, A. Mugarza, J. S. Mollejo, D. Sánchez-Portal, D. Garcia De Oteyza, M. Corso, A. Garcia-Lekue, D. Peña and J. I. Pascual, ACS Nano, 2020, 14, 1895-1901.

217 R. R. Cloke, T. Marangoni, G. D. Nguyen, T. Joshi, D. J. Rizzo, C. Bronner, T. Cao, S. G. Louie, M. F. Crommie and F. R. Fischer, J. Am. Chem. Soc., 2015, 137, 8872-8875.

218 Z. Pedramrazi, C. Chen, F. Zhao, T. Cao, G. D. Nguyen, A. A. Omrani, H. Z. Tsai, R. R. Cloke, T. Marangoni, D. J. Rizzo, T. Joshi, C. Bronner, W. W. Choi, F. R. Fischer, S. G. Louie and M. F. Crommie, Nano Lett., 2018, 18, 3550-3556.

219 S. Kawai, S. Saito, S. Osumi, S. Yamaguchi, A. S. Foster, P. Spijker and E. Meyer, Nat. Commun., 2015, 6, 8098. 
220 E. Carbonell-Sanromà, P. Brandimarte, R. Balog, M. Corso, S. Kawai, A. Garcia-Lekue, S. Saito, S. Yamaguchi, E. Meyer, D. Sánchez-Portal and J. I. Pascual, Nano Lett., 2020, 17, 50-56.

221 N. Friedrich, P. Brandimarte, J. Li, S. Saito, S. Yamaguchi, I. Pozo, D. Peña, T. Frederiksen, A. Garcia-Lekue, D. SánchezPortal and J. I. Pascual, Phys. Rev. Lett., 2020, 125, 146801.

222 P. S. Costa, J. D. Teeter, A. Enders and A. Sinitskii, Carbon, 2018, 134, 310-315.

223 C. Ma, L. Liang, Z. Xiao, A. A. Puretzky, K. Hong, W. Lu, V. Meunier, J. Bernholc and A. P. Li, Nano Lett., 2017, 17, 6241-6247.

224 C. Ma, Z. Xiao, J. Huang, L. Liang, W. Lu, K. Hong, B. G. Sumpter, J. Bernholc and A. P. Li, Phys. Rev. Mater., 2019, 3, 016001.

225 T. Marangoni, D. Haberer, D. J. Rizzo, R. R. Cloke and F. R. Fischer, Chem. - Eur. J., 2016, 22, 13037-13040.

226 J. Li, N. M. Díez, E. C. Sanromà, M. Vilas-Varela, D. G. De Oteyza, D. Peña, M. Corso and J. I. Pascual, Sci. Adv., 2018, 4, eaaq0582.

227 L. M. Mateo, Q. Sun, S. X. Liu, J. J. Bergkamp, K. Eimre, C. A. Pignedoli, P. Ruffieux, S. Decurtins, G. Bottari, R. Fasel and T. Torres, Angew. Chem., Int. Ed., 2020, 59, 1334-1339.

228 C. Ma, Z. Xiao, A. A. Puretzky, H. Wang, A. Mohsin, J. Huang, L. Liang, Y. Luo, B. J. Lawrie, G. Gu, W. Lu, K. Hong, J. Bernholc and A.-P. P. Li, ACS Nano, 2020, 14, 5090-5098.

229 X. Su, Z. Xue, G. Li and P. Yu, Nano Lett., 2018, 18, 5744-5751.

230 T. H. Vo, U. G. E. Perera, M. Shekhirev, M. Mehdi Pour, D. A. Kunkel, H. Lu, A. Gruverman, E. Sutter, M. Cotlet, D. Nykypanchuk, P. Zahl, A. Enders, A. Sinitskii and P. Sutter, Nano Lett., 2015, 15, 5770-5777.

231 Y. F. Zhang, Y. Zhang, G. Li, J. Lu, Y. Que, H. Chen, R. Berger, X. Feng, K. Müllen, X. Lin, Y. Y. Zhang, S. Du, S. T. Pantelides and H. J. Gao, Nano Res., 2017, 10, 3377-3384.

232 D. Beyer, S. Wang, C. A. Pignedoli, J. Melidonie, B. Yuan, C. Li, J. Wilhelm, P. Ruffieux, R. Berger, K. Müllen, R. Fasel and X. Feng, J. Am. Chem. Soc., 2019, 141, 2843-2846.

233 J. Li, N. Friedrich, N. Merino, D. G. de Oteyza, D. Peña, D. Jacob and J. I. Pascual, Nano Lett., 2019, 19, 3288-3294.

234 N. Pavliček, A. Mistry, Z. Majzik, N. Moll, G. Meyer, D. J. Fox, L. Gross, J. Su, M. Telychko, P. Hu, G. Macam, P. Mutombo, H. Zhang, Y. Bao, F. Cheng, Z. Huang and Z. Qiu, Sci. Adv., 2017, 12, eaav7717.

235 N. Pavliček, A. Mistry, Z. Majzik, N. Moll, G. Meyer, D. J. Fox and L. Gross, Nat. Nanotechnol., 2017, 12, 308-311.

236 S. Mishra, X. Yao, Q. Chen, K. Eimre, O. Gröning, R. Ortiz, M. Di Giovannantonio, J. C. Sancho-García, J. FernándezRossier, C. A. Pignedoli, K. Müllen, P. Ruffieux, A. Narita and R. Fasel, 2020, arXiv:2003.03577.

237 S. Mishra, D. Beyer, K. Eimre, J. Liu, R. Berger, O. Gro, C. A. Pignedoli, K. Mu, R. Fasel, X. Feng and P. Ruffieu, J. Am. Chem. Soc., 2019, 141, 10621-10625.

238 S. Mishra, D. Beyer, R. Berger, J. Liu, O. Gröning, J. I. J. I. Urgel, K. Müllen, P. Ruffieux, X. Feng and R. Fasel, J. Am. Chem. Soc., 2020, 142, 1147-1152.
239 S. Mishra, D. Beyer, K. Eimre, S. Kezilebieke, R. Berger, O. Gröning, C. A. Pignedoli, K. Müllen, P. Liljeroth, P. Ruffieux, X. Feng and R. Fasel, Nat. Nanotechnol., 2020, 15, 22-28.

240 S. Mishra, D. Beyer, K. Eimre, R. Ortiz, J. FernándezRossier, R. Berger, O. Gröning, C. A. Pignedoli, R. Fasel, X. Feng and P. Ruffieux, Angew. Chem., Int. Ed., 2020, 59, 12041-12047.

241 J. Li, S. Sanz, J. Castro-esteban, M. Vilas-varela, N. Friedrich, T. Frederiksen, D. Peña and J. I. Pascual, Phys. Rev. Lett., 2020, 124, 177201.

242 A. Candini, L. Martini, Z. Chen, N. Mishra, D. Convertino, C. Coletti, A. Narita, X. Feng, K. Müllen and M. Affronte, J. Phys. Chem. C, 2017, 121, 10620-10625.

243 B. V. Senkovskiy, M. Pfeiffer, S. K. Alavi, A. Bliesener, J. Zhu, S. Michel, A. V. Fedorov, R. German, D. Hertel, D. Haberer, L. Petaccia, F. R. Fischer, K. Meerholz, P. H. M. Van Loosdrecht, K. Lindfors and A. Grüneis, Nano Lett., 2017, 17, 4029-4037.

244 M. Mehdi Pour, A. Lashkov, A. Radocea, X. Liu, T. Sun, A. Lipatov, R. A. Korlacki, M. Shekhirev, N. R. Aluru, J. W. Lyding, V. Sysoev and A. Sinitskii, Nat. Commun., 2017, 8, 820 .

245 S. J. Heerema and C. Dekker, Nat. Nanotechnol., 2016, 11, 127-136.

246 H. Sakaguchi, Y. Kawagoe, Y. Hirano, T. Iruka, M. Yano and T. Nakae, Adv. Mater., 2014, 26, 4134-4138.

247 Z. Chen, W. Zhang, C. A. Palma, A. Lodi Rizzini, B. Liu, A. Abbas, N. Richter, L. Martini, X. Y. Wang, N. Cavani, H. Lu, N. Mishra, C. Coletti, R. Berger, F. Klappenberger, M. Kläui, A. Candini, M. Affronte, C. Zhou, V. De Renzi, U. Del Pennino, J. V. Barth, H. J. Räder, A. Narita, X. Feng and K. Müllen, J. Am. Chem. Soc., 2016, 138, 15488-15496.

248 G. Galeotti, M. Fritton and M. Lackinger, Angew. Chem., Int. Ed., 2020, 59, 22785-22789.

249 X. Y. Wang, J. I. Urgel, G. B. Barin, K. Eimre, M. Di Giovannantonio, A. Milani, M. Tommasini, C. A. Pignedoli, P. Ruffieux, X. Feng, R. Fasel, K. Müllen and A. Narita, J. Am. Chem. Soc., 2018, 140, 9104-9107.

250 V. Passi, A. Gahoi, B. V. Senkovskiy, D. Haberer, F. R. Fischer, A. Grüneis and M. C. Lemme, ACS Appl. Mater. Interfaces, 2018, 10, 9900-9903.

251 L. Martini, Z. Chen, N. Mishra, G. B. Barin, P. Fantuzzi, P. Ruffieux, R. Fasel, X. Feng, A. Narita, C. Coletti, K. Müllen and A. Candini, Carbon, 2019, 146, 36-43.

252 A. Fairbrother, J. R. Sanchez-Valencia, B. Lauber, I. Shorubalko, P. Ruffieux, T. Hintermann and R. Fasel, Nanoscale, 2017, 9, 2785-2792.

253 P. B. Bennett, Z. Pedramrazi, A. Madani, Y. C. Chen, D. G. De Oteyza, C. Chen, F. R. Fischer, M. F. Crommie and J. Bokor, Appl. Phys. Lett., 2013, 103, 253114.

254 P. Prins, F. C. Grozema, J. M. Schins, S. Patil, U. Scherf and L. D. A. Siebbeles, Phys. Rev. Lett., 2006, 96, 146601.

255 W. Sun, J. Shen, Z. Zhao, N. Arellano, C. Rettner, J. Tang, T. Cao, Z. Zhou, T. Ta, J. K. Streit, J. A. Fagan, T. Schaus, M. Zheng, S.-J. Han, W. M. Shih, H. T. Maune and P. Yin, Science, 2020, 368, 874-877. 
256 L. Liu, J. Han, L. Xu, J. Zhou, C. Zhao, S. Ding, H. Shi, M. Xiao, L. Ding, Z. Ma, C. Jin, Z. Zhang and L.-M. Peng, Science, 2020, 368, 850-856.

257 Z. Chen, H. I. Wang, J. Teyssandier, K. S. Mali, T. Dumslaff, I. Ivanov, W. Zhang, P. Ruffieux, R. Fasel, H. J. Räder, D. Turchinovich, S. De Feyter, X. Feng, M. Kläui, A. Narita, M. Bonn and K. Müllen, J. Am. Chem. Soc., 2017, 139, 3635-3638.

258 C. Vericat, M. E. Vela, G. Benitez, P. Carro and R. C. Salvarezza, Chem. Soc. Rev., 2010, 39, 1805-1834.

259 Y. Wang, Y. Zheng, X. Xu, E. Dubuisson, Q. Bao, J. Lu and K. P. Loh, ACS Nano, 2011, 5, 9927-9933.

260 L. Gao, W. Ren, H. Xu, L. Jin, Z. Wang, T. Ma, L.-P. Ma, Z. Zhang, Q. Fu, L.-M. Peng, X. Bao and H.-M. Cheng, Nat. Commun., 2012, 3, 699.

261 Q. Sun, X. Yao, O. Gröning, K. Eimre, C. A. Pignedoli, K. Müllen, A. Narita, R. Fasel and P. Ruffieux, Nano Lett., 2020, 20, 6429-6436.

262 J. Li, S. Sanz, M. Corso, D. J. Choi, D. Peña, T. Frederiksen and J. I. Pascual, Nat. Commun., 2019, 10, 200.

263 C. Moreno, M. Paradinas, M. Vilas-Varela, M. Panighel, G. Ceballos, D. Peña and A. Mugarza, Chem. Commun., 2018, 54, 9402-9405.

264 A. Rastgoo-Lahrood, M. Lischka, J. Eichhorn, D. Samanta, M. Schmittel, W. M. Heckl and M. Lackinger, Nanoscale, 2017, 9, 4995-5001.

265 K. K. Liu, W. Zhang, Y. H. Lee, Y. C. Lin, M. T. Chang, C. Y. Su, C. S. Chang, H. Li, Y. Shi, H. Zhang, C. S. Lai and L. J. Li, Nano Lett., 2012, 12, 1538-1544.
266 H. Jia, R. Yang, A. E. Nguyen, S. N. Alvillar, T. Empante, L. Bartels and P. X. L. Feng, Nanoscale, 2016, 8, 10677-10685.

267 Z. Lin, Y. Zhao, C. Zhou, R. Zhong, X. Wang, Y. H. Tsang and Y. Chai, Sci. Rep., 2015, 5, 18596.

268 J. Xia, X. Huang, L. Z. Liu, M. Wang, L. Wang, B. Huang, D. D. Zhu, J. J. Li, C. Z. Gu and X. M. Meng, Nanoscale, 2014, 6, 8949-8955.

269 M. Chen, R. C. Haddon, R. Yan and E. Bekyarova, Mater. Horiz., 2017, 4, 1054-1063.

270 J. Kang, D. Shin, S. Bae and B. H. Hong, Nanoscale, 2012, 4, 5527-5537.

271 Y. Zhang, Y. Zhang, G. Li, J. Lu, X. Lin, S. Du, R. Berger, X. Feng, K. Müllen and H. J. Gao, Appl. Phys. Lett., 2014, 105, 023101.

272 D. Han, Q. Fan, J. Dai, T. Wang, J. Huang, Q. Xu, H. Ding, J. Hu, L. Feng, W. Zhang, Z. Zeng, J. M. Gottfried and J. Zhu, J. Phys. Chem. C, 2020, 124, 5248-5256.

273 D. G. De Oteyza, A. García-Lekue, M. Vilas-Varela, N. MerinoDíez, E. Carbonell-Sanromà, M. Corso, G. Vasseur, C. Rogero, E. Guitián, J. I. Pascual, J. E. Ortega, Y. Wakayama and D. Peña, ACS Nano, 2016, 10, 9000-9008.

274 L. Wang, H. Dong, Z. Guo, L. Zhang, T. Hou and Y. Li, J. Phys. Chem. C, 2016, 120, 17427-17434.

275 J. Liu, B. W. Li, Y. Z. Tan, A. Giannakopoulos, C. SanchezSanchez, D. Beljonne, P. Ruffieux, R. Fasel, X. Feng and K. Müllen, J. Am. Chem. Soc., 2015, 137, 6097-6103.

276 H. Sakaguchi, S. Song, T. Kojima and T. Nakae, Nat. Chem., 2017, 9, 57-63. 